\title{
Review
}

\section{Fluorescent glycoconjugates and their applications}

Cite this: DOI: $10.1039 / x 0 \times x 00000 x$

Received 00th January 2019,

Accepted 00th January 2019

DOI: $10.1039 / x 0 x x 00000 x$

www.rsc.org/

\author{
Baptiste Thomas, ${ }^{a 1}$ Kai-Cheng Yan, ${ }^{b 1} \mathrm{Xi}$-Le Hu, ${ }^{b}$ Marion Donnier-Maréchal, ${ }^{a}$ Guo- \\ Rong Chen, ${ }^{b *}$ Xiao-Peng He ${ }^{b *}$ and Sébastien Vidal ${ }^{a *}$
}

Glycoconjugates and their applications as lectin ligands in biology has been thoroughly investigated in the past decades. Meanwhile, the intrinsic properties of such multivalent molecules were limited essentially to their ability to bind to their receptors with high selectivity and/or avidity. The present review will focus on multivalent glycoconjugates displaying an additional capability such as fluorescence properties for applications toward imaging of cancer cells, detection of proteins or pathogens but also for drug delivery systems toward targeted cancer therapy. This review is a collection of research articles discussed in the context of the structural features of the fluorescent glycoconjugates organized according to their fluorescent core scaffold and with their representative applications.

\section{Introduction}

Carbohydrates play a critical role in the signalling pathways and communication of cells during infections by viruses or bacteria and in cancer. ${ }^{1}$ Nature utilizes multivalency to reach the necessary level of avidity between carbohydrate-binding proteins (e.g. lectins) and their respective oligosaccharidic binding epitopes. This phenomenon has been coined as the "glycoside cluster effect"2, 3 and has triggered numerous designs of multi-glycosylated molecular architectures. ${ }^{4-13}$ These pieces of work provided useful and critical data to ascertaining the parameters that govern the affinity towards carbohydratebinding proteins of therapeutic interest. ${ }^{14-16}$ The present review will focus on the design and applications of fluorescent glycoconjugates with either monosaccharides or glycoclusters. The fluorescent moiety will notably be used as the multifunctional core scaffold for the construction of a glycocluster. Fluorescent dyes can also be conjugated onto a (oligo)saccharide core towards specific applications.

Fluorescence is a phenomenon by which a substance emits light upon absorbance of light or other electromagnetic irradiations. Because of the simplicity and sensitivity of fluorescence techniques, the development of fluorescent probes has been an active research area in environmental science, biochemistry, chemical biology and biomedicine. ${ }^{17-19}$ Fluorescent small molecules, polymeric and supramolecular carbohydrate derivatives have been developed for biological sensing and bio-imaging. ${ }^{20}$, 21 Traditional strategies for the design of fluorescent probes involve the fluorescence "on-off" (the fluorescence signal is quenched upon interaction with the analyte), "off-on" (the quenched fluorescence signal of a probe is reactivated upon interaction with the analyte) and ratiometric (the fluorescence emission band is shifted upon interaction with the analyte) rationales. Aggregation-induced-emission (AIE) has also been recognized as a promising strategy to design fluorescent "offon" type (or fluorogenic) probes for biosensing and bioimaging. ${ }^{22-25}$ Contrary to the conventional aggregation-causedquenching ( $A C Q$ ), AIE is a process by which weak-emitting fluorophores show a much stronger fluorescence emission upon aggregation due to a restriction of intramolecular rotation or vibration (RIR or RIV). ${ }^{26,27}$ Most recently, a new concept of vibration-induced emission (VIE) described a serendipitously discovered photophysical property of $N, N^{\prime}$-disubstituteddihydrodibenzo[a,c]phenazines. The fluorescence emission of these VIEgens can reversibly shift between the blue and red region when the microenvironment they exist, such as temperature, polarity and viscosity, is changed. ${ }^{28-37}$

The present review will be divided according to the different fluorophores to which carbohydrates are conjugated, producing structurally and functionally diverse fluorescent glycoclusters. Representative examples of polymeric backbones used for building multivalent fluorescent glycoconjugates are also summarized. The design principles as well as the applications of the developed glycoconjugates will be discussed while the synthetic strategy will not be discussed in detail.

\section{Small-molecular fluorescent glycoconjugates}

\subsection{Tetraphenylethylene-based glycoconjugates}

Tetraphenylethylene (TPE) is a prototypical RIR-based AIEgen, which has been extensively used to construct fluorogenic probes and imaging agents due to their drastic fluorescence enhancement upon aggregation. TPE is composed of four phenyl rings that are linked to a central ethene rod through a single bond. TPE has merits in terms of its high emission efficiency, facile synthesis, accessible structural modification and stability among other AIE-active materials. ${ }^{38}$ It is reported that 
the conjugation of a specific ligand, such as a carbohydrate, significantly improves the selectivity of the imaging probe toward a specific biological target. In recent years, studies have discovered a wide range of lectins, toxins, pathogens and enzymes which can be sensitively detected by fluorescencebased techniques with TPE-based glycoconjugates, which were mainly synthesized by the $\mathrm{Cu}(\mathrm{II})$-catalyzed azide-alkyne 1,3dipolar cycloaddition (CuAAC click reaction). Its fluorescence color can be tuned by controlling the intramolecular rotation of the TPE's phenyl rings. Indeed, due to the free rotation of the four phenyl rings around the ethene linker, the nonplanar TPE is non-emissive in a dissolved state. However, in aggregates (or solid state) the intramolecular rotation of theses phenyl rings is confined, reducing non-radiative relaxation, and hence resulting in strong photoluminescence.

The sensing of concanavalin A (ConA) using mannosylated TPEbased glycoclusters was reported (Figure 1). ${ }^{39}$ Four or eight copies of mannopyranoside, which can be selectively recognized by ConA, were incorporated to a TPE scaffold, producing three glycoclusters with good water solubility and, thus a low fluorescence emission. Subsequently, the addition of ConA congregated the glycoclusters through multivalent mannose binding, leading to a fluorescence "turn-on" response due to the AIE properties of TPEs. Other unselective proteins used did not cause the emission to increase, suggesting that the lectin sensing was selective.
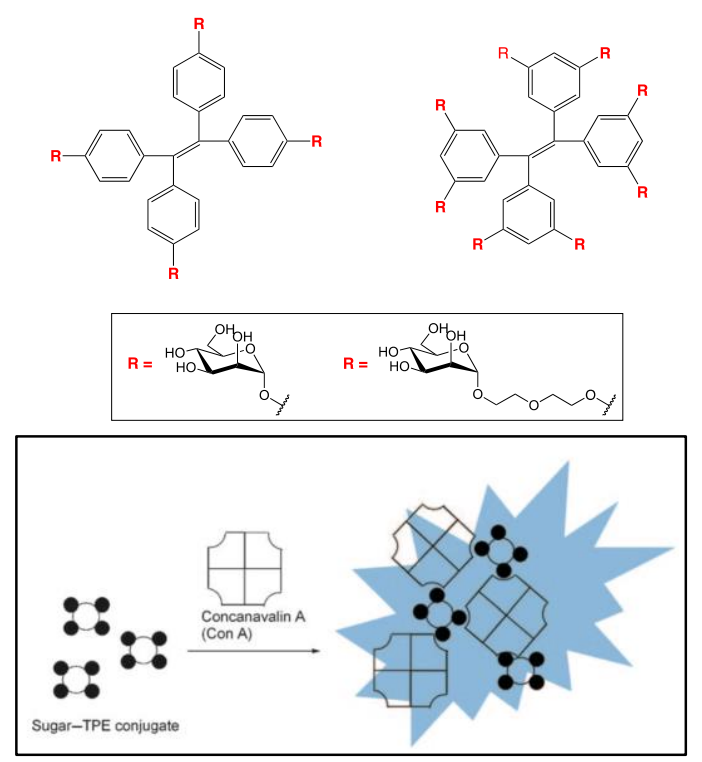

Figure 1 Mannosylated TPEs and schematic representation of a "turn-on" fluorescent sensor for ConA based on aggregation-induced emission (AIE). Reproduced from ref. ${ }^{39}$ with permission from the Wiley-VCH, copyright 2010.

Using the same rationale, the authors also achieved the sensing of non-lectin proteins ${ }^{40}$ through a fluorescence-quenching based assay. In that study, they used ConA as an "aptamer" to construct a complexed probe with the TPE glycoclusters. The TPE-based glycoclusters displayed a strong emission in complex with ConA because of aggregation (Figure 2). The subsequent addition of other proteins led to disaggregation of the glycocluster-ConA complexes through a competitive interaction, thereby turning off the AIE fluorescence.
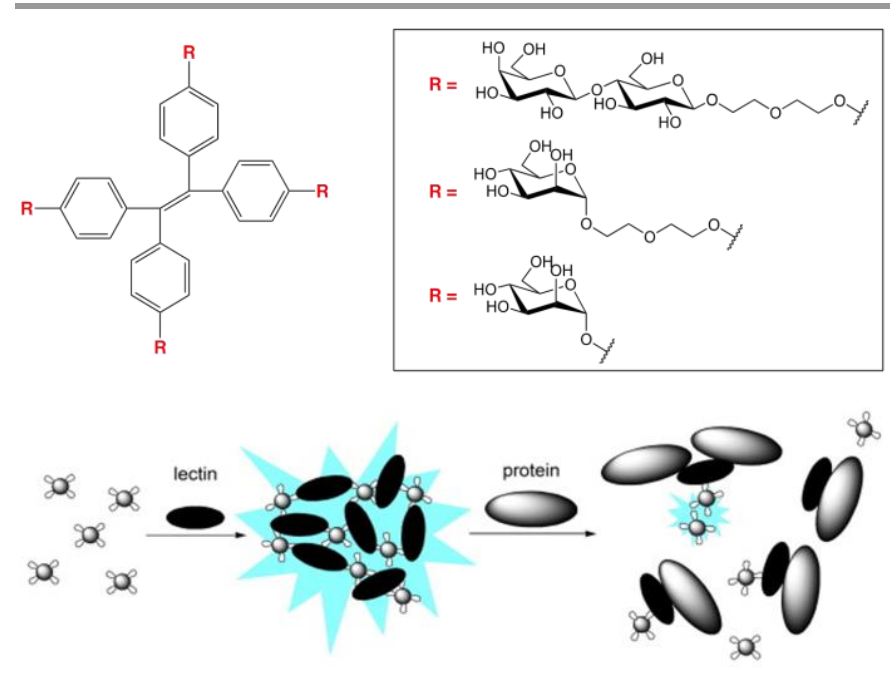

turn-on

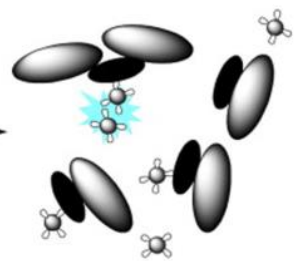

turn-off

Figure 2 Structures of glycosylated TPEs and schematic illustration of the displacement assay for detection of protein-protein interactions based on AIE. Reproduced from ref. ${ }^{40}$ with permission from the Elsevier B.V., copyright 2010.

Detection of toxins was also achieved using a lactosylated TPE glycoconjugate as a fluorogenic sensor for the detection of cholera toxin (CT) (Figure 3). ${ }^{41}$ The TPE-based glycoconjugate developed has been shown to bind CT with high sensitivity, producing a fluorescence "turn-on" response through AIE. This could lead to future applications in the rapid diagnosis of cholera, although the drawback of the short emission wavelength of TPE that might cause false-positive signals in realistic biological samples must still be overcome.
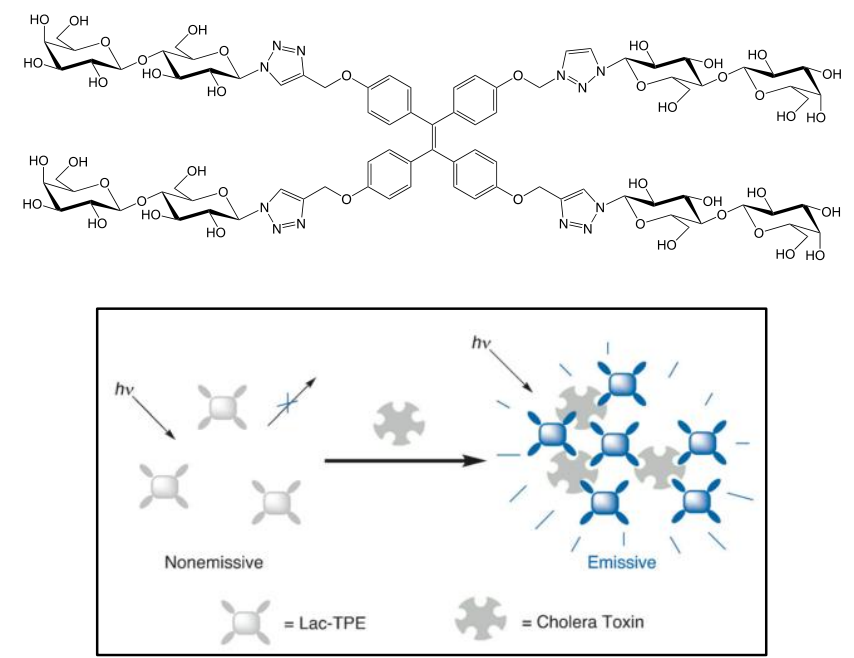

Figure 3 Lactosylated TPEs and schematic illustration of fluorescence "turn-on" assay for CT based on AIE effect. Reproduced from ref. ${ }^{41}$ with permission from the Wiley-VCH, copyright 2011.

Two TPE-based fluorogenic probes (sialyl- $\alpha-2,6$-lactose-TPE and a control Lac-TPE without the sialoside epitope) coupled with two different oligosaccharides were applied for the fluorescence "turn-on" detection of influenza viruses (Figure 4). ${ }^{42}$ The binding specificity to influenza virus of the glyco-probes was first tested by dot blot with two types of lectins RCA120 (Ricinus communis agglutinin 120) binding to lactose and SSA 
(Sambucus sieboldiana agglutinin) which selectively binds sialyl- $\alpha-2,6-G a l / G a l N A c$. Therefore, a human influenza virus selectively binds to $\alpha-2,6-S L-T P E$ (but not Lac-TPE) with the terminal $\alpha$-2,6-sialioside through its hemagglutinin (HA) expressed at high density on its surface. The AIE fluorescence was activated by the binding of the virus to $\alpha-2,6-S L-T P E$. Consequently, this study offers an effective tool for the detection of influenza viruses with fluorescence "turn-on" response.

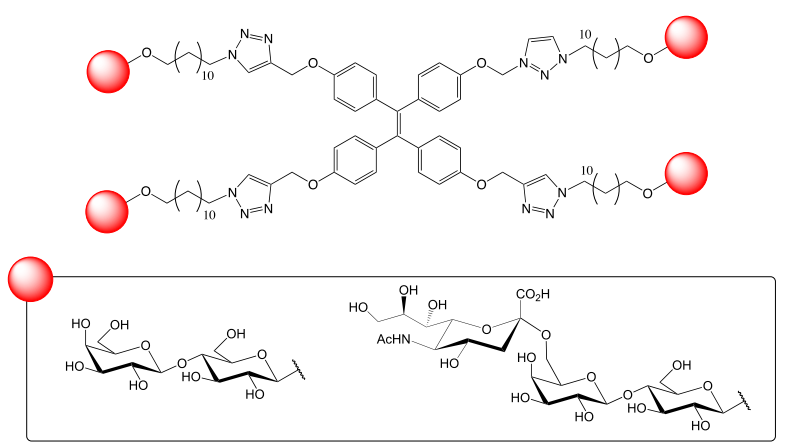

Figure 4 Lactosylated and sialylated TPEs.

TPE-Based glycoclusters bearing either galactoside, fucoside, mannoside or glucoside have been reported with the aim to develop anti-adhesive therapeutics against Pseudomonas aeruginosa infection (Figure 5). ${ }^{43}$ While the affinity for two bacterial lectins (LecA and LecB) was greatly improved through the multivalency of the glycoclusters, their anti-adhesive properties could not be verified in a cell adhesion assay. Nevertheless, these glycoclusters displayed AIE properties in water but again their detection in a fluorescent cell adhesion assay was not possible due to overlapping signal with the bacteria's intrinsic fluorescence.
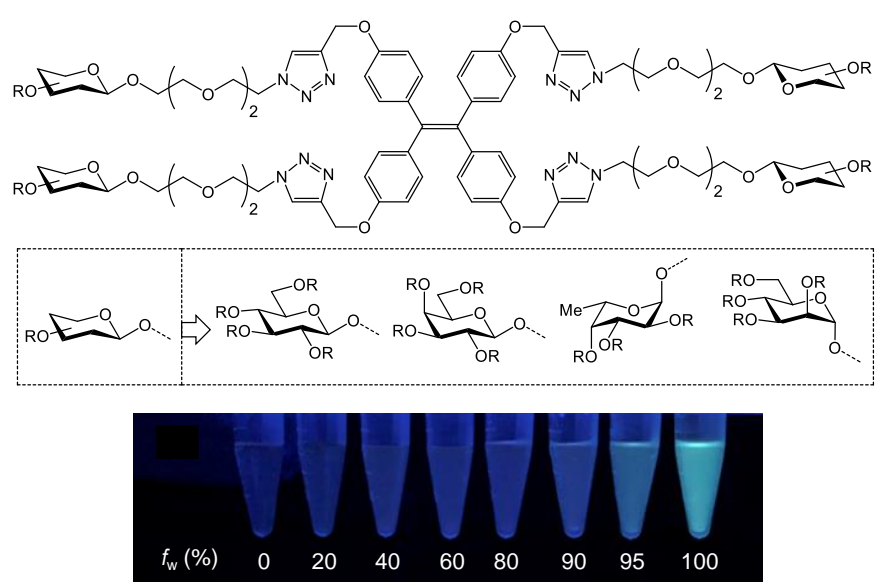

Figure 5 TPE-based glycoclusters and their fluorescent AIE properties. Reproduced from ref. ${ }^{43}$ with permission from the Royal Society of Chemistry, copyright 2018.

In addition to the sensing of lectins, TPE-based glycoclusters were described for the detection of $\beta$-glucosidase, an important member of the cellulose-decomposing enzymatic system (Figure 6). ${ }^{44}$ Cellulose was conjugated with TPE to detect this enzyme. While the probe was initially weakly fluorescent because of the good dispersibility in aqueous solution, the addition of the $\beta$-glucosidase depolymerized the hydrophilic cellulose from the AIE core. The TPE molecules therefore aggregated in solution, leading to a largely enhanced fluorescence for the sensitive detection of the enzymatic activity. The probes developed have also been applied for lectin detection through the same AIE-based sensing mechanism.

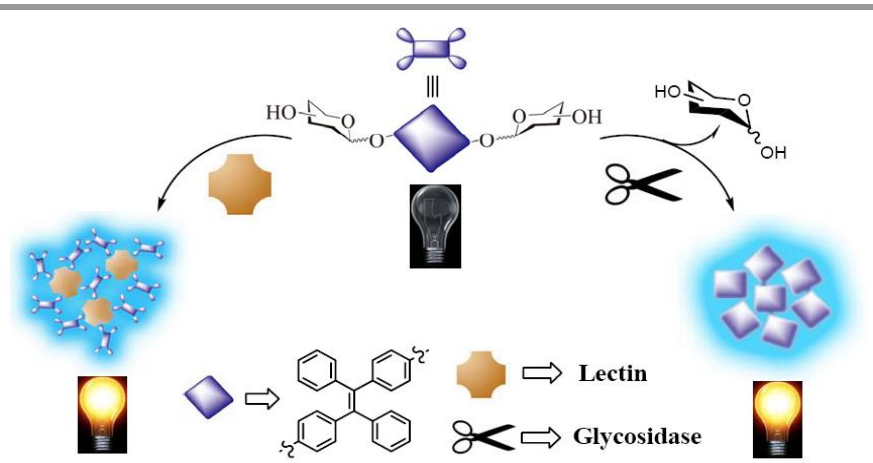

Figure 6 Illustration of cellulose depolymerization upon addition of $\beta$-glucosidase leading to aggregation of the TPE-cellulose glycoconjugates as fluorescent probes based on the AIE effect. Reproduced from ref. ${ }^{44}$ with permission from the Royal Society of Chemistry, copyright 2011.

TPE-based glycoclusters displaying GalNAc (N-acetylgalactosamine) epitopes were reported as a high-affinity ligand for the human macrophage galactose-binding C-type lectin (MGL) whose inhibition is of therapeutic interest. ${ }^{45}$ Nine glycoclusters with carbohydrates conjugated on different multivalent templates including one TPE have been prepared (Figure 7), among which S-glycosides have the advantage to resist hydrolysis compared to native $O$-glycosides. Interestingly, although the TPE-based glycocluster was a rare example among the tested molecules, it displayed the strongest binding with the lectin in the series.

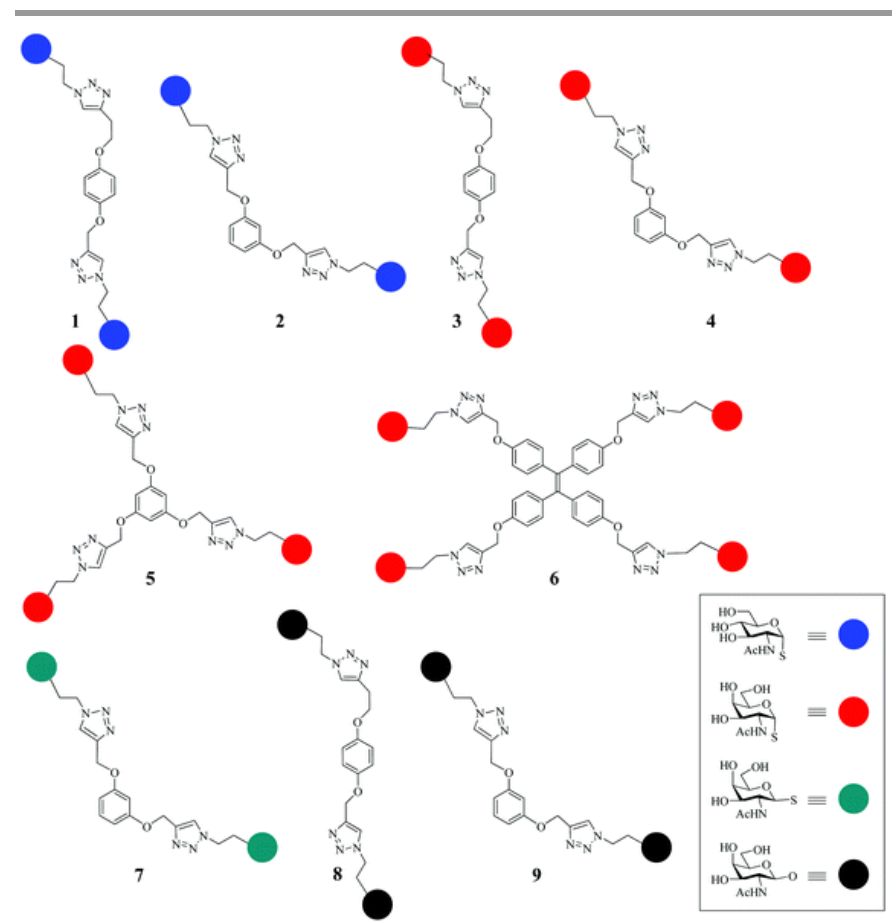

Figure 7 Structure of different glycoclusters as MGL ligands. Reproduced from ref ${ }^{45}$ with permission from the Royal Society of Chemistry, copyright 2015. 
TPE glycoclusters were designed for a traceable drug delivery system for imaging the drug distribution in cells (Figure 8). ${ }^{46} \mathrm{~A}$ dextran-hydrazone-TPE was synthesized which could selfassemble into micelles, allowing the effective loading of the anticancer drug doxorubicin (DOX). The drug-loaded micelles exhibited the typical blue TPE fluorescence because of aggregation. Upon endocytosis by cells, the TPE fluorescence could be used to track drug distribution through fluorescence microscopy. Moreover, the TPE emission could be further enhanced intracellularly due to a more hydrophobic aggregation of the TPE molecules through the cleavage of the acid-labile hydrazone bond on dextran in endosomes and lysosomes.

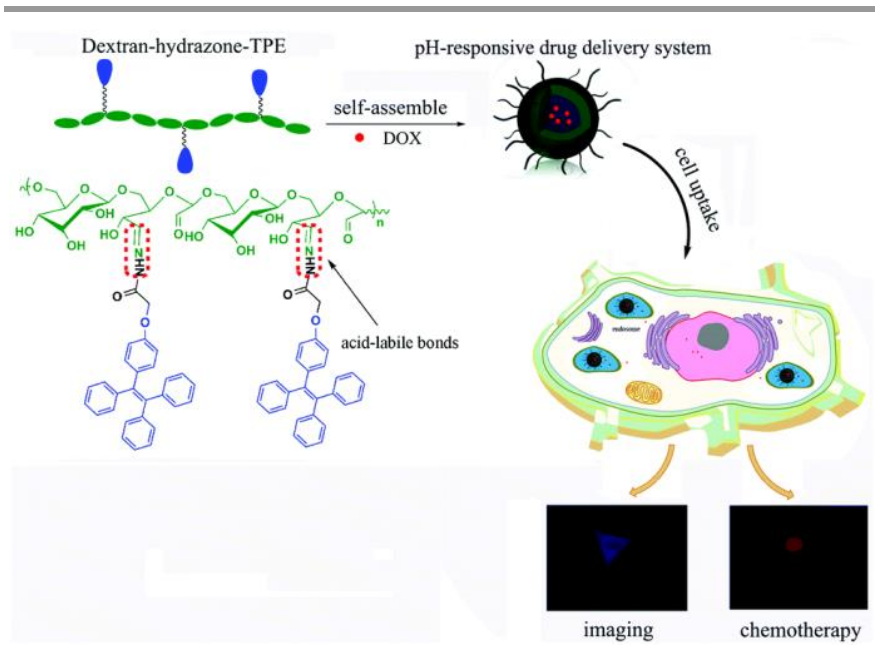

Figure 8 Drug loaded micelle with AIE properties as a theranostic platform for intracellular imaging and cancer treatment. Reproduced from ref. ${ }^{46}$ with permission from the Royal Society of Chemistry, copyright 2015. Note: The 3-position of the pyranose units should appear as equatorial (instead of axial) to match the stereochemistry of D-glucose (and not D-allose).

A colloidal fluorescent nanoparticle capable of encapsulating TPE molecules through polydopamine coating was then further functionalized by galactosides to target specific cancer cells (Figure 9). ${ }^{47}$ The dopamine's catechol moiety was conjugated (Figure 9b) with the galactosylated thiol (Figure 9a) to afford glyconanoparticles with or without chitosan. The results indicated that the galactose-functionalization led to the selective imaging of HepG2 (human hepatoma) cells over-expressing the asialoglycoprotein receptor (ASPGr; galactose-selective). Moreover, under physiological conditions, the fluorescence of the glyconanoparticles was determined to be stable. This suggests the potential of AIE-based glyco-materials for targeted fluorescence imaging of cancer cells, although AIEgens with an emission wavelength across the near-infrared (NIR) region would be desired for in vivo imaging to minimalize biological auto-fluorescence.
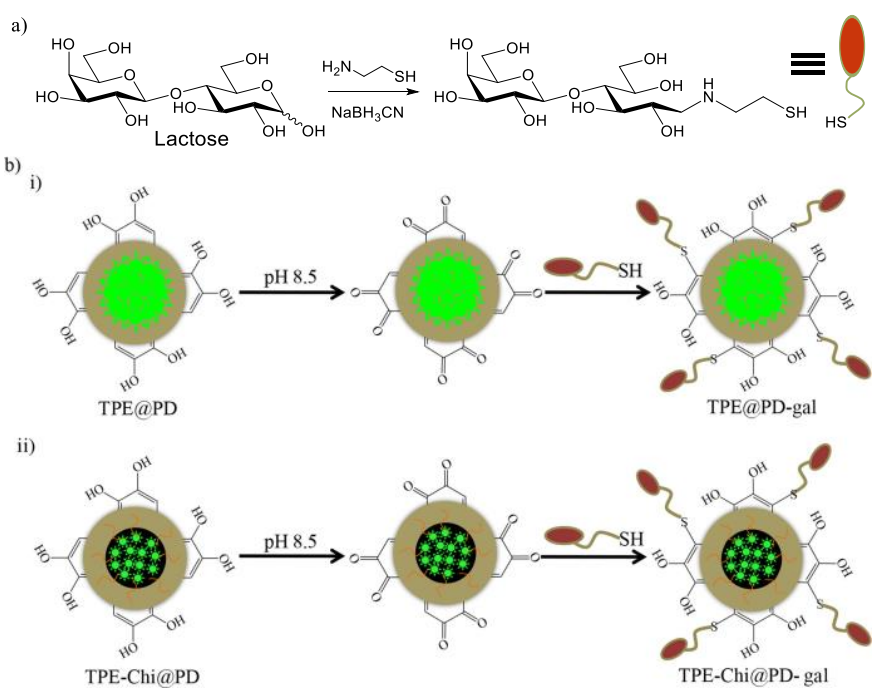

Figure 9 Functionalization of polydopamine-coated TPE-based glyconanoparticles for targeted cell imaging. Reproduced from ref. ${ }^{47}$ with permission from the American Chemical Society, copyright 2018.

Apart from the molecules based on TPE, we would like to shortly discuss another glyco-diketopyrrolopyrroles (glyco-DPPs) since they also display AIE properties just like TPE derivatives. Carbohydratelectin recognitions were investigated using this molecule, and the recognition-induced fluorescence "turn-on" was based on the AIE property. ${ }^{48}$ The molecules were also made by "click" reaction (Figure 10), and when the specific lectin was added, a significant emission increase was observed. The selectivity was also verified since one glyco-DPP could selectively recognize ConA through the mannosides while the other could only recognize peanut agglutinin (PNA) using its galactoside epitopes.

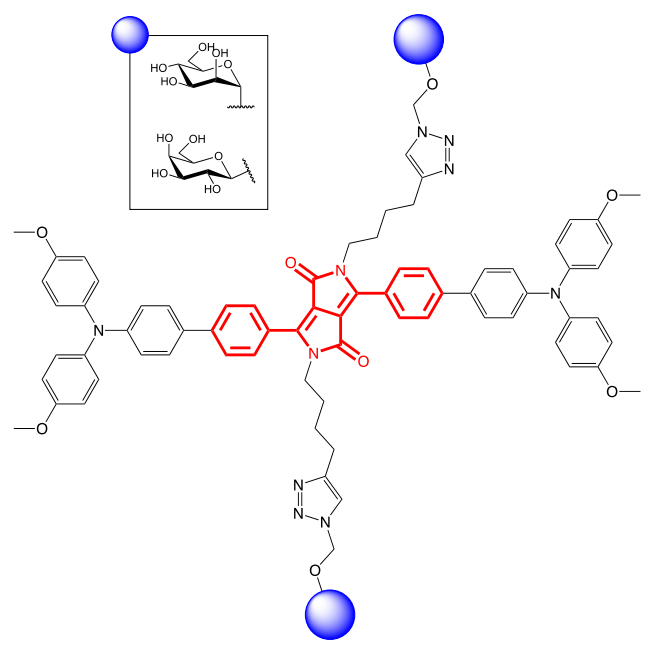

Figure 10 Structure of the AIE-based DPP dyes.

The carbohydrate epitopes could also be conjugated both on the diketopyrrolopyrrole and the triphenylamine peripheral moieties through CuAAC to afford the hexa-substituted glycol-DPP (Figure 11). ${ }^{49}$ This NIR emissive glycoconjugate could monitor the concentration dependent detection of lectins based on AIE properties triggered by the aggregation of the probes win the present of the 
multivalent lectins. This probe could be applied to the imaging of HepG2 cells through interactions with the galactose-specific ASGPr (Asialoglycoprotein Receptor).

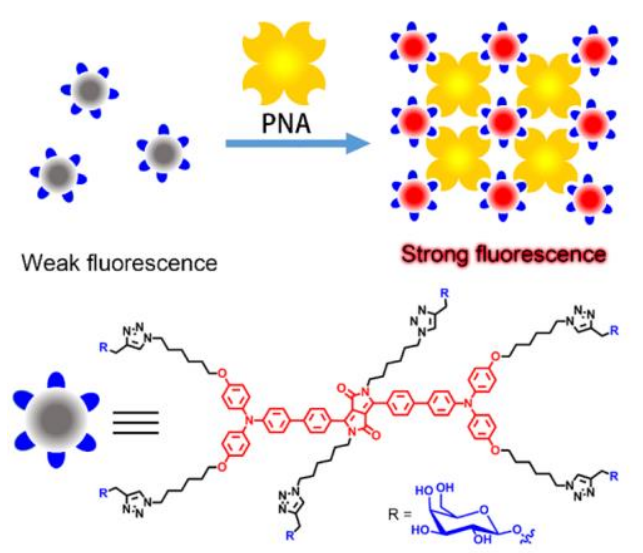

Figure 11 Hexa-functionalized DPP and application for the detection of lectins. Reproduced from ref. ${ }^{49}$ with permission from the Springer-Verlag $\mathrm{GmbH}$, copyright 2018.

\subsection{Perylenediimide-based glycoconjugates}

Perylenediimide (PDI) is characterized by notable thermal and photochemical stabilities as well as suitable fluorescence quantum yields both in organic solvents and water. ${ }^{50,51}$ These properties have been exploited in several applications such as field effect transistors ${ }^{50,52-55}$ or other biologically relevant systems. ${ }^{56-59}$ The fluorescent core of the PDI can be functionalized with different patterns based on its bay substituents and/or the imide moieties providing up to six conjugation sites. The PDI-glycoconjugates are also synthesized mainly by CuAAC and are widely studied for applying in a range of biomedical areas.

Mannosylated PDI-based glycoclusters with high watersolubility were reported focusing on their synthesis, optical properties and binding interactions with ConA. ${ }^{60}$ In fluorescence detection there was a significant quenching and a small blue shift of the probe's emission upon addition of ConA.

Another PDI-based glycosylated polymer interaction with ConA was reported. ${ }^{61}$ Compared with the former hexamannosylated PDI-based glycocluster, this compound displayed as much as 18 mannoside epitopes on six branching points (Figure 12). The inhibition of binding to ConA was carried out using the monovalent ConA ligand methyl $\alpha-D-$ mannopyranoside (MMP) in a competition experiment.

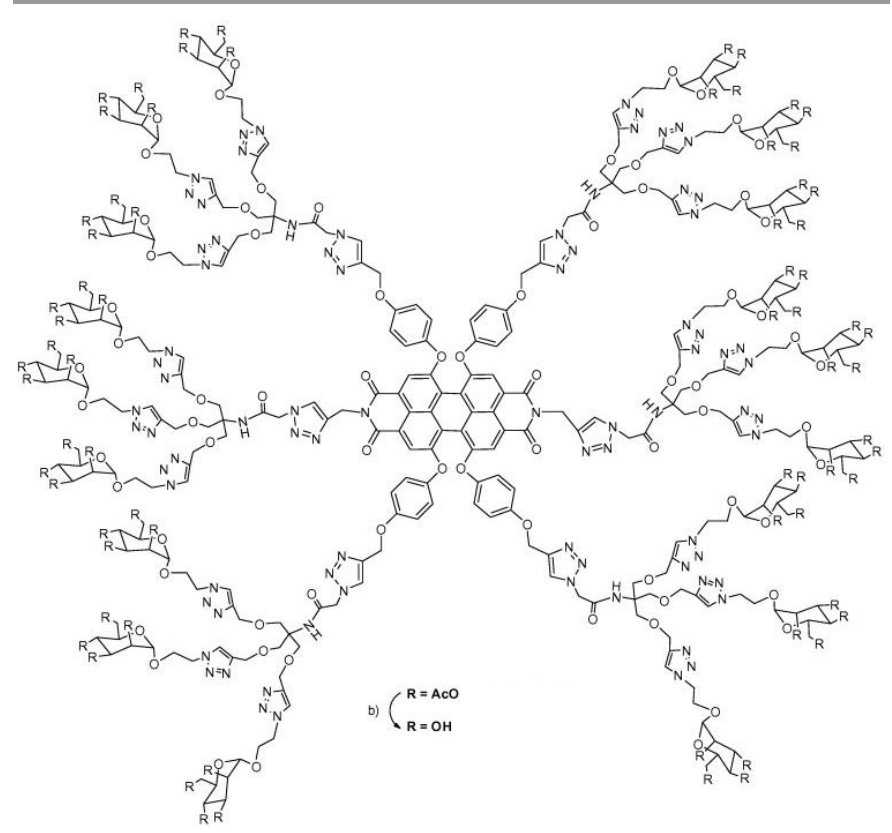

Figure 12 Structure of PDI-18-Man. Reproduced from ref. ${ }^{61}$ with permission from the Elsevier B.V., copyright 2013.

The same group ${ }^{62}$ reduced the number of mannosides to 12 in a newly synthesized PDI-based glycocluster. Fluorescence "offon" phenomenon was demonstrated upon addition of ConA while non-selective lectin such as PNA or BSA (bovine serum albumin, not a lectin) were inactive. The interaction with macrophage-like cell lines (highly mannosylated on their surface) of the PDI-based glycocluster was then investigated leading to another "turn-on" fluorescence, and the MMP inhibition talked above could be directly studied by cell-imaging. Another similar study from this group ${ }^{63}$ reported another 18-valent mannosylated PDI-based glycocluster for bioimaging of murine macrophage cells. Clearly, these two latter studies have extended the applications of PDI-based glyco-probes to biomedical areas.

The most significant use of PDI-based glycoclusters in cell imaging was reported through their combination with dicyanomethylene $4 \mathrm{H}$-pyran (DCM) probes and assembled through non-covalent interactions ( $\pi-\pi$ stacking) into particles. Liu and co-workers ${ }^{64}$ reported self-assembled glyco-dots from PDIbased glycoclusters and their use in selective cancer cell imaging (Figure 13). The galactosylated glyco-dots PDI-Gal could selectively visualize human liver cancer cell (HepG2) expressing galactose-binding ASGPr with help of DCM fluorophore, while the nanoparticle with mannosides PDI-Man could only recognize breast cancer cell (MDA-MB-231) expressing mannose receptors. Human cervical cancer (HeLa) and lung cancer (A549) cell lines without the galactose or mannose receptor expression were used as controls (Figure 14). Also, the glycodots could release fluorescence DCM molecules upon addition of serum proteins (HSA and BSA) rationalizing the release mechanism of the DCM dye inside cells. 

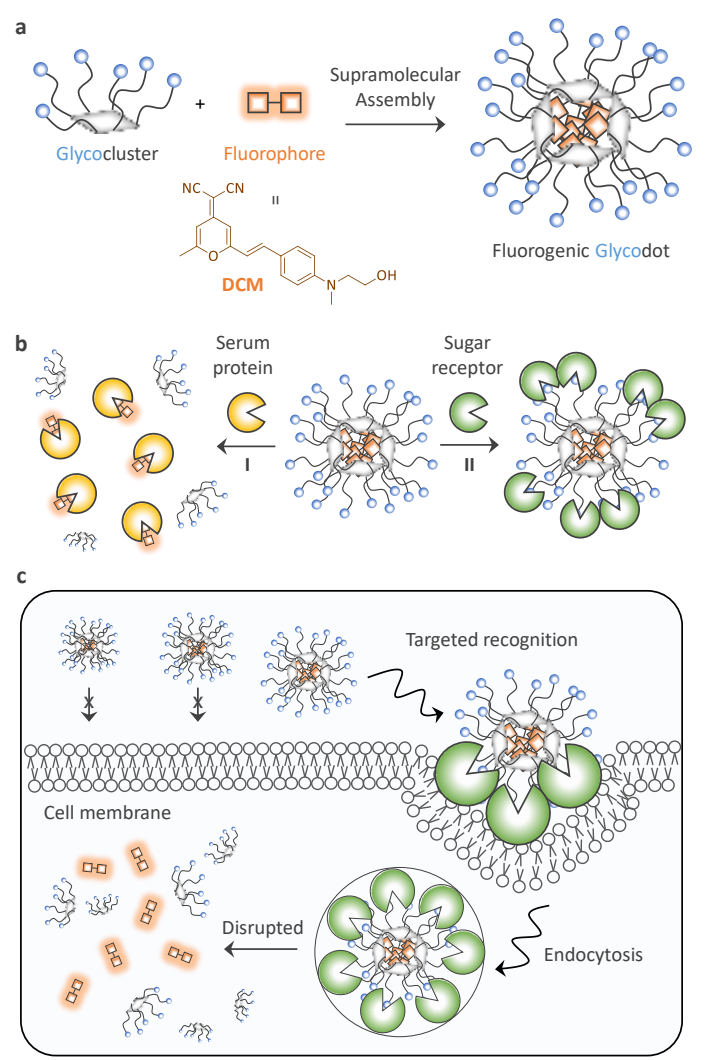

Figure 13 (a) Supramolecular assembly of fluorogenic glyco-dots. (b) The release of DCM molecules and the ligand-receptor recognition. (c) Targeted imaging of cells expressing carbohydrate receptors. Reproduced from ref. ${ }^{64}$ with permission from the Royal Society of Chemistry, copyright 2017.
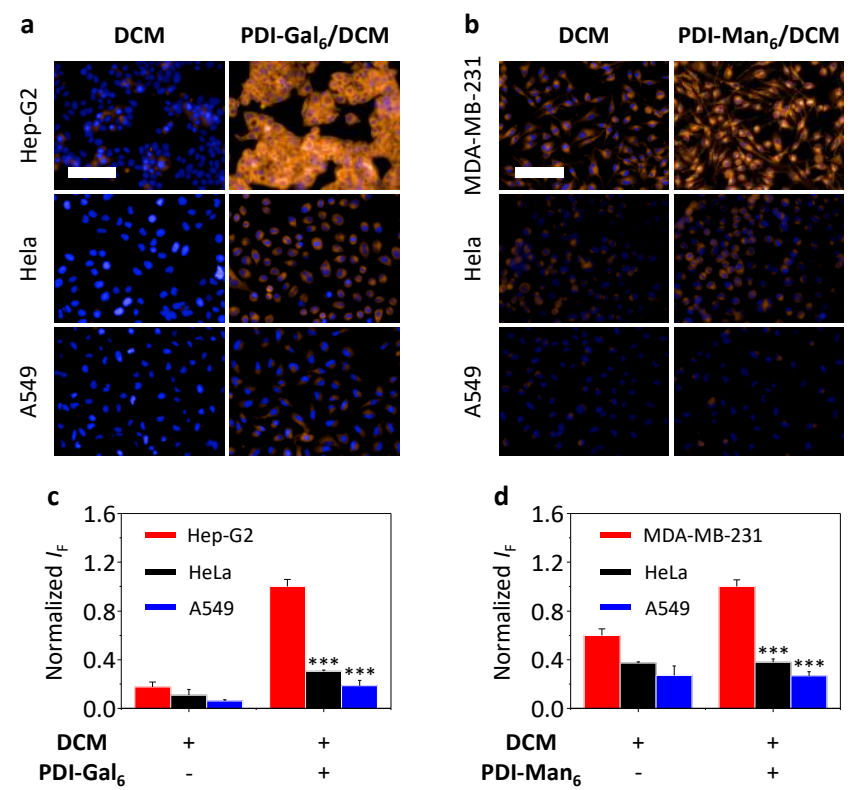

Figure 14 Fluorescence imaging and quantification of different cancer cells with DCM and two perylenediimide-based probes. Reproduced from ref. ${ }^{64}$ with permission from the Royal Society of Chemistry, copyright 2017.
In addition to the sensing of cells, some studies also mentioned sensing and treating of bacteria. A fluorescent glycosylated polymer containing PDI was synthesized for application in the conjugation with Escherichia coli and cell-imaging. ${ }^{65}$ The glycoconjugate could bind to the $E$. coli upon incubation and display strong emission when clustering at the surface of the bacteria (Figure 15).
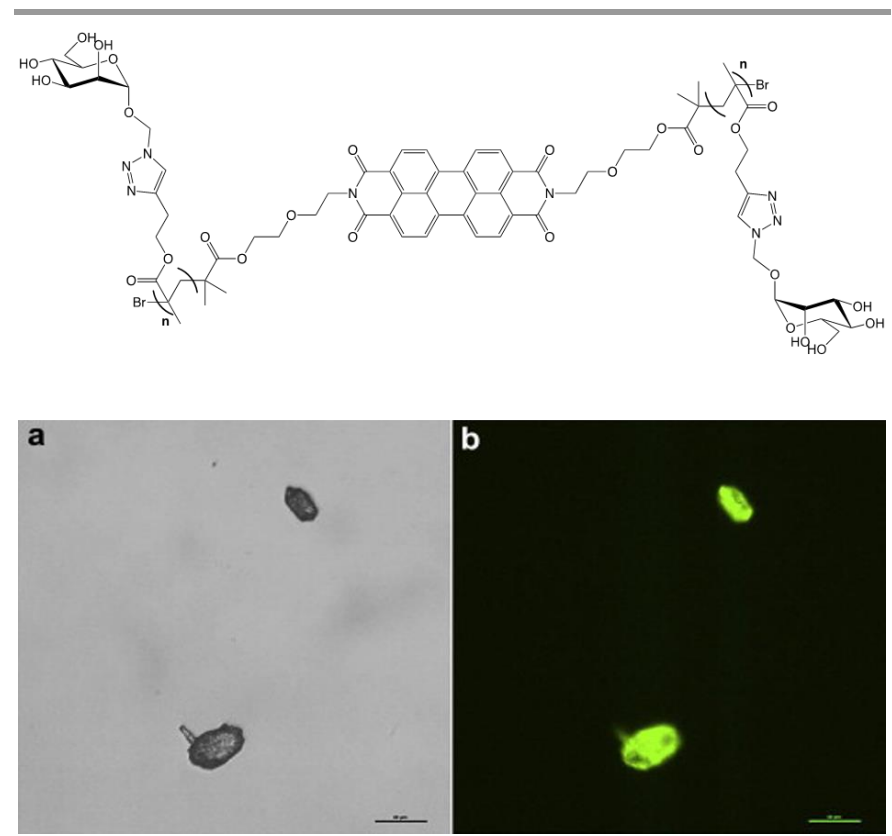

Figure 15 Structure of a PDI-based glycosylated polymer probe and the fluorescence-stained bacterial clusters using the polymer. Reproduced from ref. ${ }^{65}$ with permission from the Elsevier B.V., copyright 2011.

E. coli expresses the type 1 fimbrae adhesin FimH (selective for $\alpha$-mannosides) and aggregation of the bacteria and fluorescence staining of the complexes were observed after the addition of the glyco-probes. Such green bacterial clusters could be fully revealed and visualized by a fluorescent microscope. Then the following results indicated the low cytotoxicity (high cellviability) of the glyco-probes, as well as their cell-imaging ability, similar to former reports. Strong green fluorescence of the probe was observed in all three cells involved in the study (macrophages, 3T3 fibroblasts and KB cells), which demonstrated their large range applications in cell-imaging (Figure 14).

The antibacterial applications of PDI-based glycoconjugates have also been reported by our group. ${ }^{66}$ Tetravalent and hexavalent glycoclusters based on PDI core scaffolds bearing galactose, glucose, mannose, and fucose were synthesized. Their evaluation as LecA and LecB ligands, two lectins proteins involved in the adhesion of Pseudomonas aeruginosa to host cells, was achieved by isothermal titration calorimetry (ITC) $\left(K_{d}\right.$ in the nanomolar range) and cell adhesion assays. The PDIbased glycoclusters were proved to be anti-adhesive agents against $P$. aeruginosa.

Besides the binding properties of PDI-based glycoconjugates to lectins and cells, some studies focused on their self-assembly physico-chemical properties in water. Chiral helical selfassembly of lactosylated PDI-based glycoclusters ${ }^{67}$ provided right-handed helical supramolecular structure held together through $\pi-\pi$ stacking generating modified fluorescence emis- 
sion. The resulting multivalent helical supramolecular glycoconjugate exhibited specific binding with PNA through its lactoside epitopes (Figure 16) while ConA and BSA had no binding. The results of self-assembly study clearly indicated that the glycoconjugate existed non-aggregated in DMSO. Addition of water induced the self-aggregation through strong $\pi-\pi$ stacking interactions.

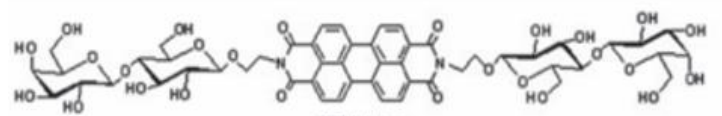

PBI-Lac
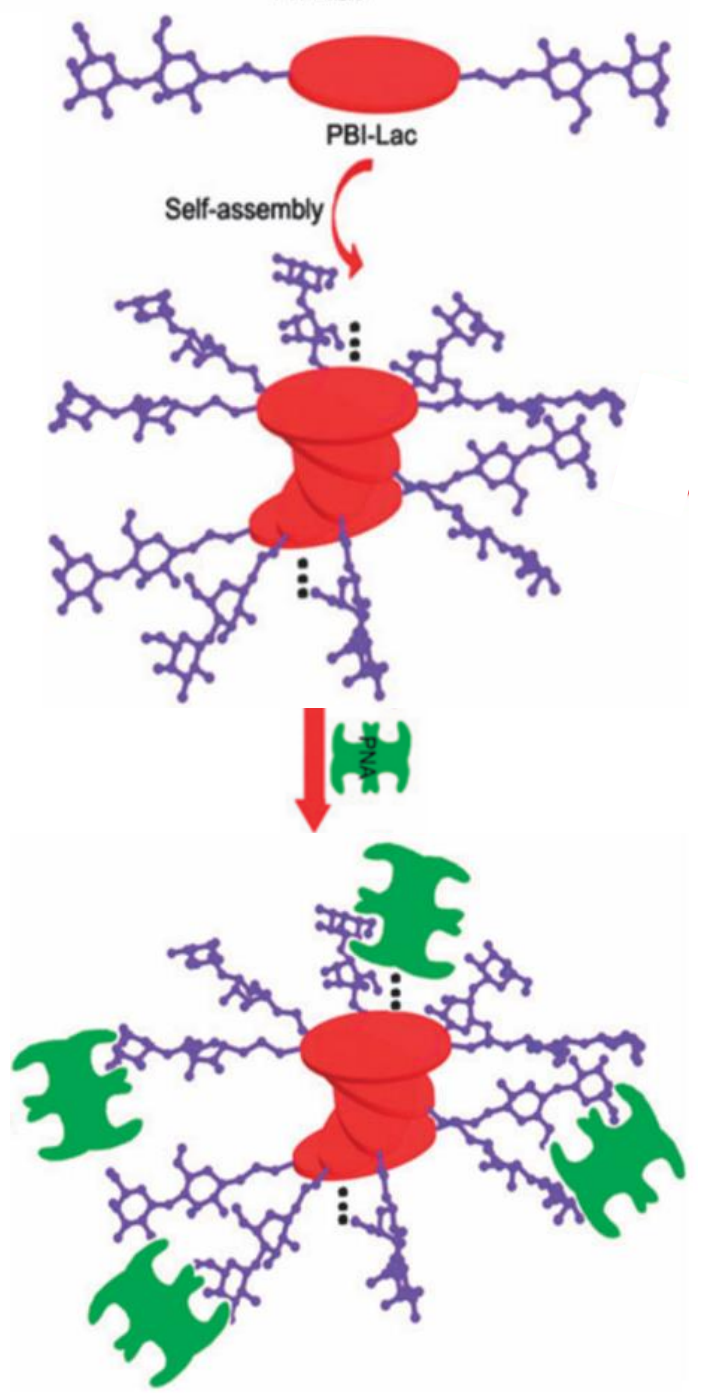

Figure 16 Structural illustration of PDI-Lac displaying the helical self-assembly mode and its interaction with PNA. Reproduced from ref. ${ }^{67}$ with permission from the Royal Society of Chemistry, copyright 2012.

Another study as demonstrated that similar mannosylated PDIbased glycoclusters could also generate chiral self-assemblies as nanoparticle with a left-handed helical conformation. ${ }^{68}$ Because the terminal groups were mannosides instead of lactosides, the specific binding interaction with ConA was studied (instead of PNA). The supramolecular chirality of PDIbased glycoclusters and its relationship with the type of carbo- hydrates were further investigated ${ }^{69}$ through eight heterosubstituted PDI-based glycoclusters (Figure 17).

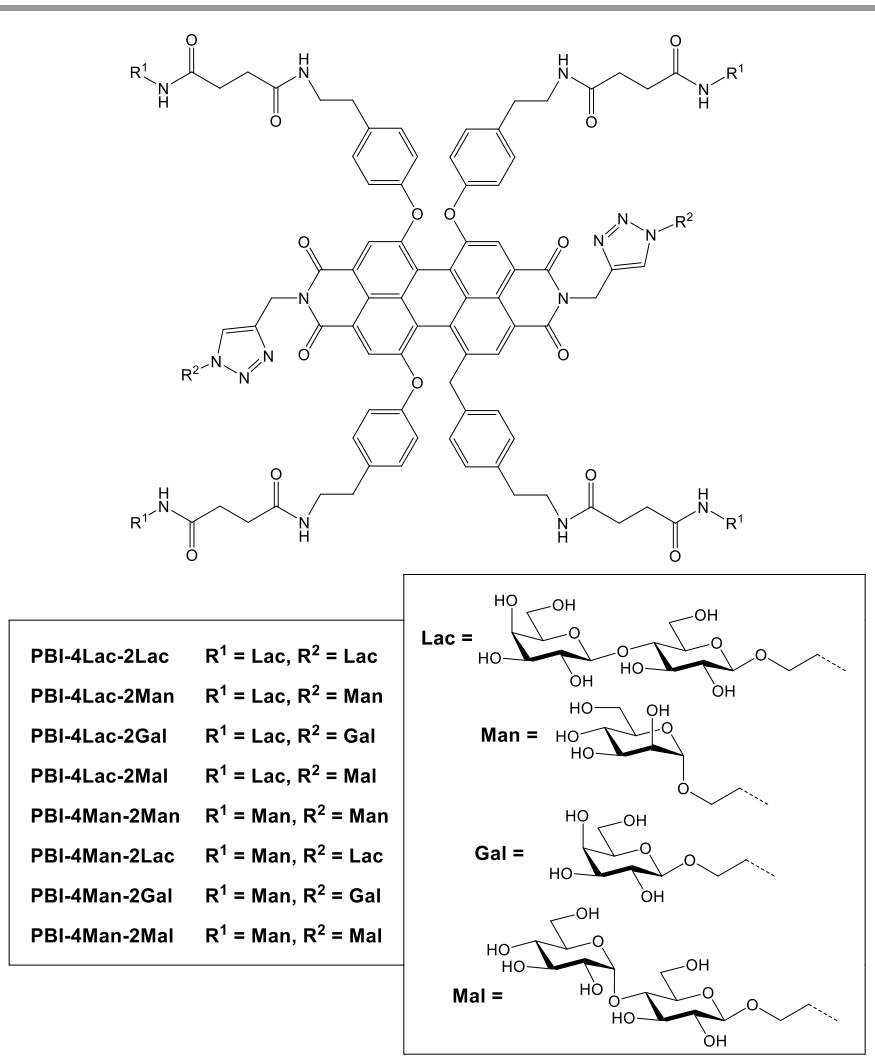

Figure 17 Structures of hetero-substituted PDI-based glycoclusters. Reproduced from ref. ${ }^{69}$ with permission from the Wiley-VCH, copyright 2015.

The influence of temperature was evaluated and noticed for mannosylated and galactosylated PDI-based glycoclusters with a modification of the stacking angle while lactosylated and maltosylated derivatives were much less sensitive to the increase of temperature. This lower difference in supramolecular chirality induced with disaccharides (lactose and maltose) was rationalized by the imide substituents generating steric effects to modify this stacking angle but the additional hydrogen bonds between the disaccharide residues would compensate this deformation much more than for monosaccharides (mannose and galactose). Also, $\mathrm{H}$-bond interactions between carbohydrates in the bay regions induced the formation of the supramolecular helical self-assembly.

Hexavalent PDI-based glycoconjugates with both amide and 1,2,3-triazole "click" linkages led to controllable supramolecular chirality of their self-assembled nanoparticles. ${ }^{70}$ One glycoconjugate showed right-handed chirality while the others showed left-handed chirality. This study indicated that the chirality also can be influenced by the covalent linkages between glycosides and the PDI core.

An amphiphilic PDI-based glycoconjugate dyad molecule and its assembling properties in different solvents ${ }^{71}$ was studied and compared using a range of methods to detect and characterize the aggregated helical structures (Figure 18). The stacking was very significant in water (WAT), while unclear stacking occurred in octane (OCT). The reason might be that nonlayered supramolecular structures were formed in OCT while in WAT, layered supramolecular aggregates were constructed 
through $\pi-\pi$ stacking. Also, controls had been studied, showing that the molecule almost remained dispersed in solvents such as tetrahydrofuran (THF) and chloroform ( $\mathrm{CHL})$.

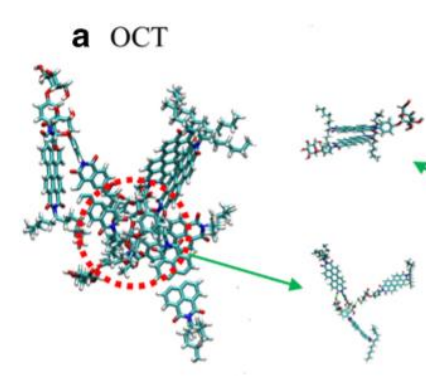

c $\mathrm{THF}$
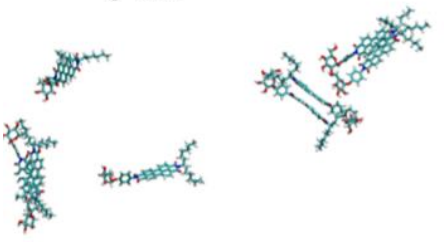

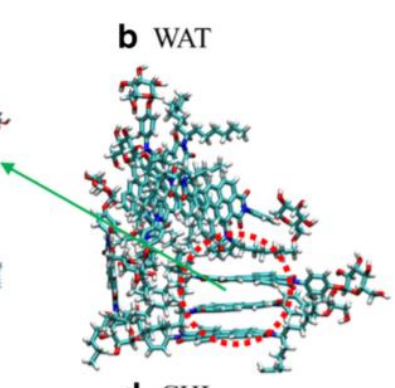

d $\mathrm{CHL}$

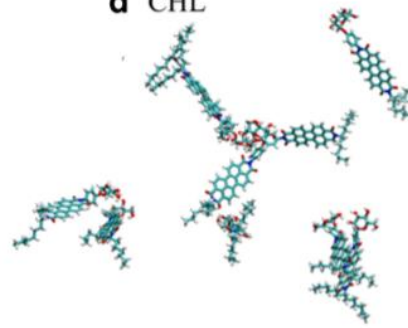

Figure 18 Supramolecular self-assembly of PDI-HAG in (a) solvent (OCT) and (b) water (WAT), as well as (c,d) control solvents ( $T H F$ and $\mathrm{CHL}$ ). Reproduced from ref. ${ }^{71}$ with permission from the Springer-Verlag $\mathrm{GmbH}$, copyright 2018.

Another interesting report indicated the H-type aggregation from their high excimer-state emission of a de-symmetrized PDI derivative, different from the symmetric structures discussed above and containing only one carbohydrate (lactose) appendage. ${ }^{72}$ This study was applied for the visualization of a fingerprint, using the emission properties of the PDI moiety. This technique allowed the observation of a red latent fingerprint under daylight. An even more significant fingerprint image could be observed after irradiation of red fluorescence at $365 \mathrm{~nm}$ without an emission filter (Figure 19).
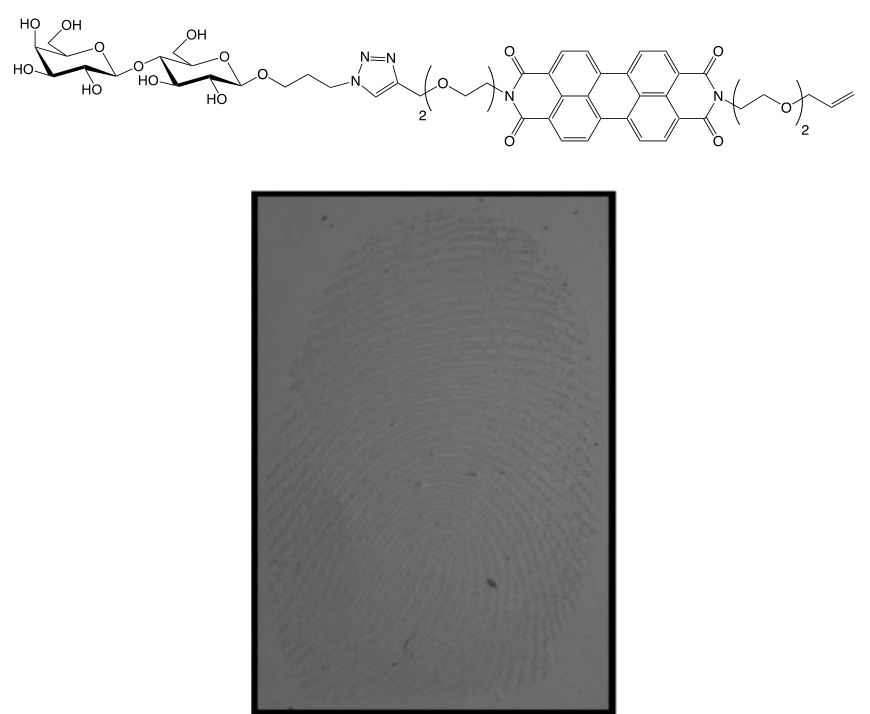

Figure 19 Lactosylated de-symmetrized perylene bisimide derivative. Fingerprint image viewed in daylight on a PVDF membrane transferred from a glass slide. Reproduced from ref. ${ }^{72}$ with permission from the Wiley-VCH, copyright 2015.

\subsection{1,8-Naphthalimide-based glycoconjugates}

Relevant reports about fluorescence applications of glycoconjugates based on 1,8-naphthalimide (NI) can also be summarized such as DNA targeting, potent anticancer activity and cellular imaging agents. ${ }^{73-77}$ The $\mathrm{NI}$ is particularly attractive due to its convenient derivatization strategy and versatile fluorescence modulating mechanisms. NIs are easily synthesised from the corresponding 1,8-naphthalic anhydrides by reaction with an amine. Substitution in the aromatic ring can have a major effect on the chemical, photochemical and spectroscopic properties of the NI. The resulting 1,8-naphthalimides are strongly fluorescent, with an emission in the green part of the electromagnetic spectrum and can be shifted towards the red by altering the nature of both the ring substituent and the imide site. Glycosylated NI-based probes were used to detect thiophenol in water, as a toxic pollutant for fish. ${ }^{78}$ The probe showed specific response to thiophenol and was also proved to be eligible for monitoring traces in river water samples (Figure 20).

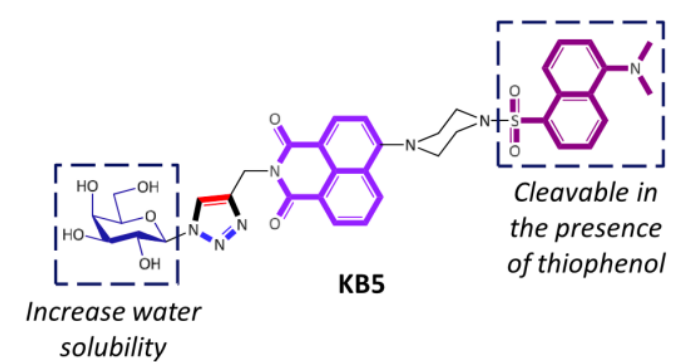

Figure 20 Glycosylated NI-based probe to detect thiophenol in water. Reproduced from ref. ${ }^{78}$ with permission from the Elsevier B.V., copyright 2015.

In addition, the galactosylated probe-metal complex was used to analyze sulfide ion $\left(\mathrm{S}^{2-}\right)$ in water. ${ }^{79}$ The $\mathrm{S}^{2-}$ anion is an environmental pollutant released from the manufacturing industries, and it is also produced by microbial reduction of sulfate by anaerobic bacteria or meat proteins, inducing negative effects. Selective and sensitive detection was accomplished using the glycoprobe-Cu(II) complex which showed a specific “off-on" response to $\mathrm{S}^{2-}$ in a displacement assay (Figure 21).

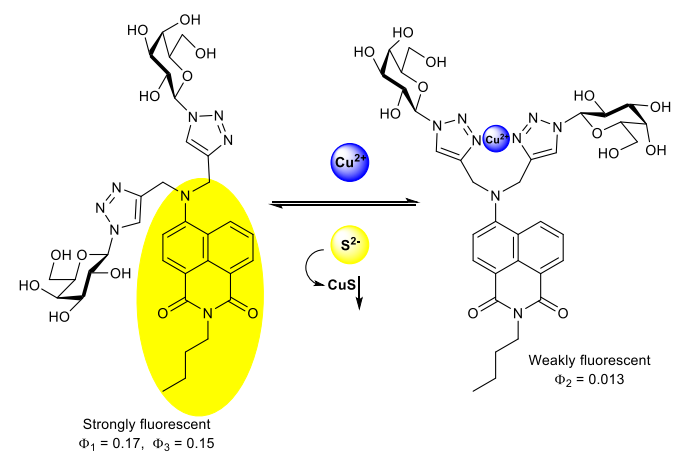

Figure 21 Proposed displacement mechanism for fluorescence "off-on" sensing of sulfide anions. Reproduced from ref. ${ }^{79}$ with permission from the Elsevier B.V., copyright 2017. 
The $\mathrm{NI}$ core could also be used to detect proteins ${ }^{80}$ using the foldable glyco-probes displaying two kinds of carbohydrate epitopes, which could crosslink with lectins (ConA and PNA). The folded structure did not display fluorescence properties and upon binding to lectins, the unfolded conformation would generate a fluorescence signal in an "off-on" FRET (fluorescence resonance energy transfer) system. After binding to lectins, the NI-based glycoconjugates were then unfolded and constructed into "hand-in-hand" supramolecular complexes (Figure 22). The intracellular receptor-targeting fluorescence imaging was also conducted using the constructed probe LKB2 and LKB5 in several cancer cells, indicating the effective LKB2 entering in HepG2 cells with galactose-specific receptor ASGPr expressing (Figure 23).

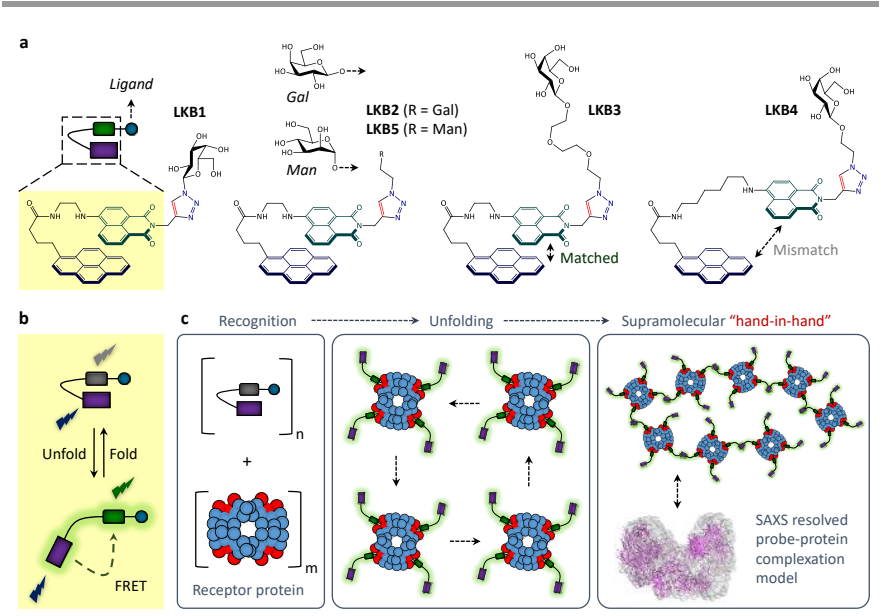

Figure 22 Illustration of (a) structures of the foldable probes. (b) The folding and unfolding process. (c) The recognition and assembling of probes to proteins. Reproduced from ref. ${ }^{80}$ with permission from the Royal Society of Chemistry, copyright 2016.

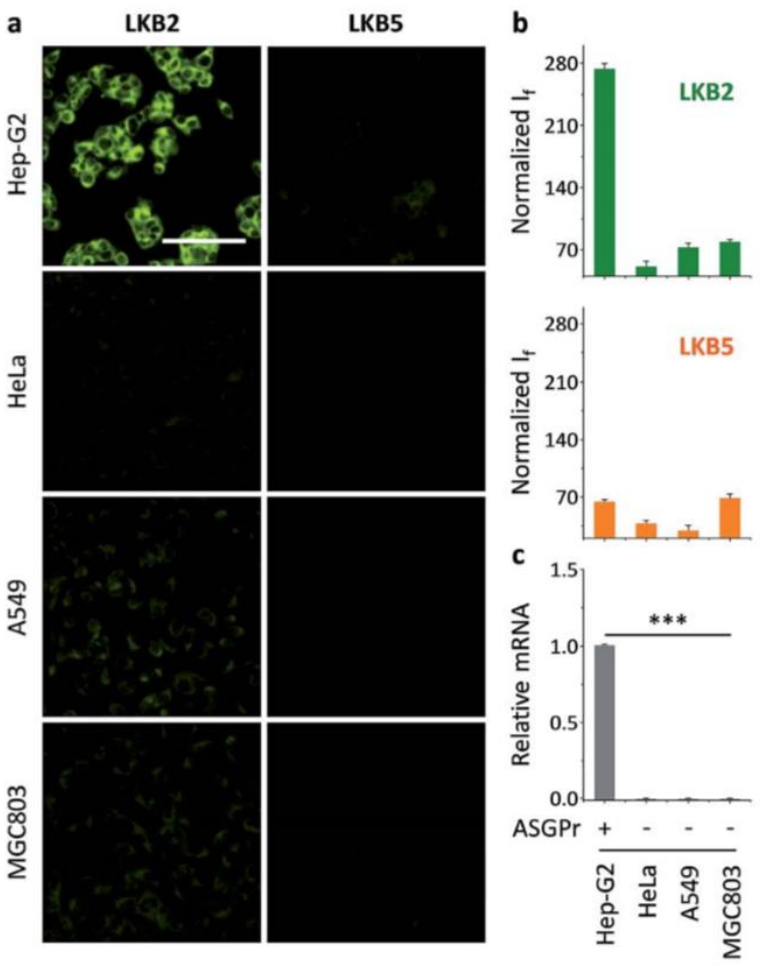

Figure 23 Fluorescence imaging in HepG2 cancer cells with ASGPr and control cells using probes LKB2 (galactose conjugated) and LKB5 (mannose conjugated), quantification of fluorescence in four cells and relative mRNA level of different cancer cells. Reproduced from ref. ${ }^{80}$ with permission from the Royal Society of Chemistry, copyright 2016.

Foldable NI-based glycoconjugates could also sense a virus in an instantaneous identification of the receptor-binding specificity of Influenza $A$ viruses. ${ }^{81}$ The foldable glycoconjugate probe similar to the former design was produced and can be unfold in the presence of influenza virus (Figure 24). Significantly, the original fluorescence emission of the folded probes was weak, but the emission became much stronger in the presence of the virus. Thus, in homogeneous solution, the glycan-receptor specificity of Influenza A virus can be easily and sensitively monitored by fluorescence detecting methods. 

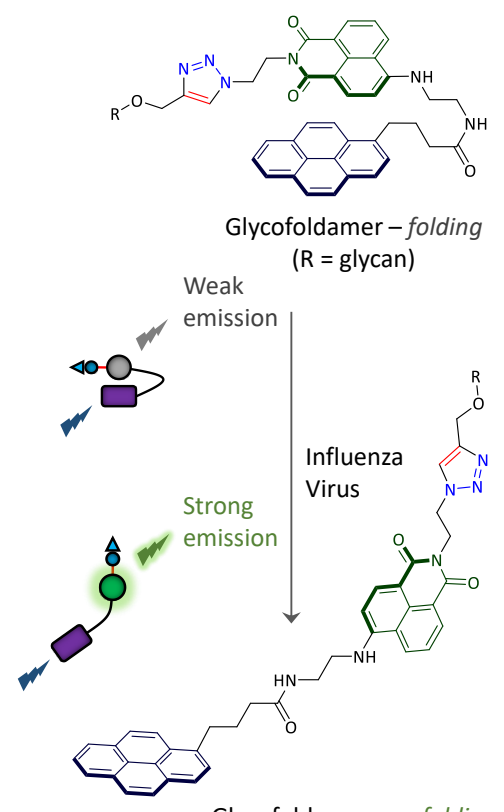

Glycofoldamer - unfolding

$$
\text { ( } R=\text { glycan) }
$$

Figure 24 Folded probes could unfold to fluoresce upon interaction with an influenza virus. Reproduced from ref. ${ }^{81}$ with permission from the Wiley-VCH, copyright 2016

Application for the tumor cell imaging of NI-based fluorescent glyco-probes was also demonstrated through the monitoring of glycosidases activity. ${ }^{82}$ The NI-based fluorescent probes were released from their glycosylated "pro-probes" precursors under the action of glycosidase removing the carbohydrate residues through hydrolysis. The glycosylated "pro-probes" could not enter the cells, but after glycosidase-catalyzed removal of the carbohydrate moieties, the resulting "probes" could enter the tumour cells (HeLa cells in cervical cancer, HepG2 and colon carcinoma HCT-116). Thus, a flexible cell imaging system controlled by glycosidase could be realized (Figure 25).

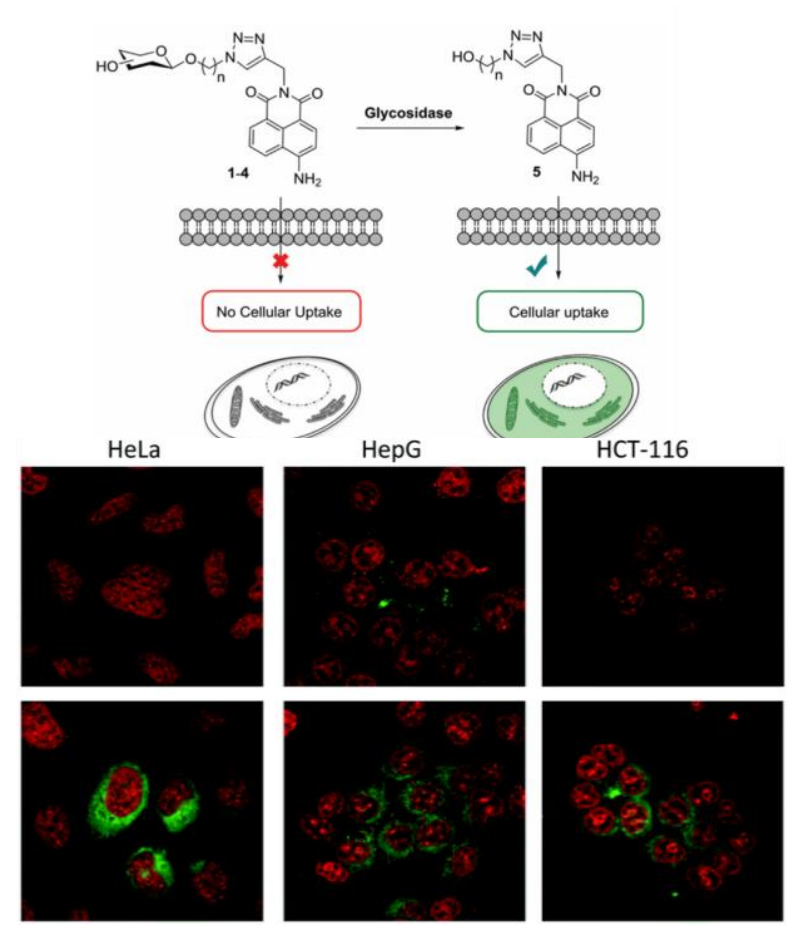

Figure 25 Illustration of the controlled cancer cell-imaging system using "proprobe" $\mathbf{1}$ in three cancer cells to be activated into resulting probe. Reproduced from ref. ${ }^{82}$ with permission from the Royal Society of Chemistry, copyright 2016.

Cyclodextrin(CD)-based thin-layer $\mathrm{MnO}_{2} 2 \mathrm{D}$ probes with a $\mathrm{NI}$ fluorescent moiety and carbohydrate epitopes for specific cell targeting were designed for imaging of cancer cells. ${ }^{83}$ The mannosylated and galactosylated epitopes were first conjugated to the $\mathrm{NI}$ scaffold and then functionalized with an adamantane moiety. Polymers functionalized with $C D$ will then interact with the adamantly moieties through interactions in the $C D$ hydrophobic cavity to provide glyco-dots. Further association with $2 \mathrm{D} \mathrm{MnO}_{2}$ led to the fluorogenic 2D probes though grafting of glyco-dots onto the $2 \mathrm{D} \mathrm{MnO}_{2}$ material. In liver cancer cell and triple-negative breast cancer cell imaging assays, the carbohydrate moieties could recognize the receptors on the cell surface and then enter the cells. The peptide GSH (glutathione) which is abundant in cancer cells reduced the $\mathrm{Mn}(\mathrm{IV}) \mathrm{O}_{2}$ into $\mathrm{Mn}(\mathrm{II})$, thus releasing the glyco-dots into cells, inducing the fluorescence recovery and realizing the cancer cell imaging (Figure 26). The liver cancer cells HepG2 and triple-negative breast cancer cells MDA-MB-231 were used because they have ASGPr and mannose receptors which could selectively recognize galactosides and mannosides respectively. 


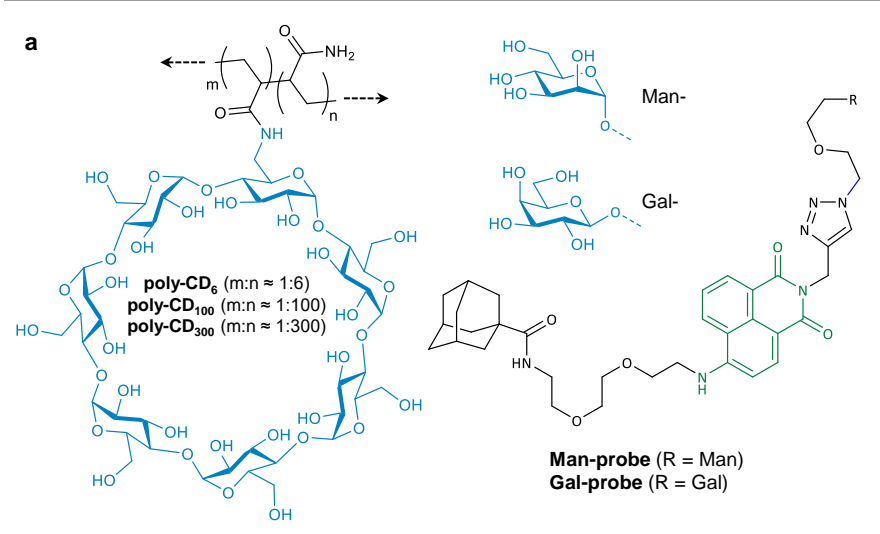

b
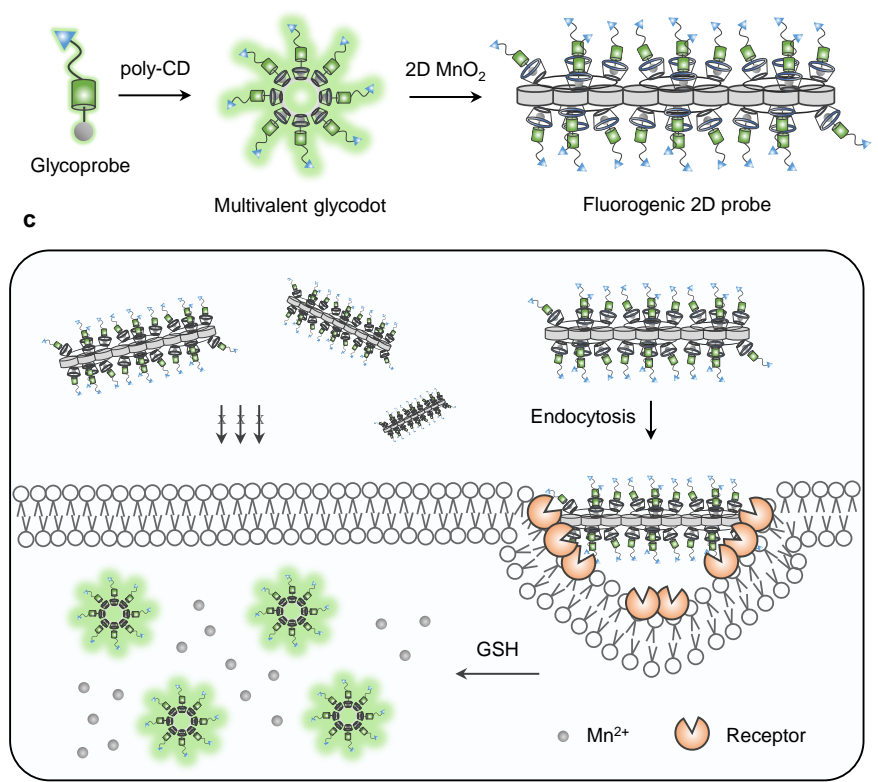

Figure 26 (a) Structures of the CD-based fluorescent glycoconjugates. (b) Illustration of the assembling and aggregation to the $2 \mathrm{D}$ probe. c) Schematic process for cell imaging. Reproduced from ref. ${ }^{83}$ with permission from the Royal Society of Chemistry, copyright 2018.

Another self-congregated probe based on gold nanoparticles (AuNPs) as well as CD as the assembling core has been reported for theranostics. ${ }^{84}$ The structure of the single probe was similar, and the carbohydrate moiety linked to the probe was also a galactoside. The whole nanoparticle could also further aggregate, in the presence of PNA, inducing a fluorescence increase as well as the release of reactive oxygen species (ROS). The targeted cell imaging was conducted successfully with HepG2 cells (Figure 27). Moreover, this work also studied the drug delivery and photodynamic properties of such glycoconjugates. The anticancer drug hydroxycamptothecin (HCPT) was incubated with the AuNPs for the drug delivery assay. The results showed that after mixing with AuNPs, the effect and selectivity of the drug increased dramatically, killing cancer cells along with the toxic ROS produced upon light guided by the fluorescence monitoring. Further evaluation was conducted to support this, showing that after the illumination of the drug-AuNPs hybrid with red-light, the anticancer effect further increased. Cell-death inducing anticancer effect of the intracel-

lular ROS produced by AuNPs under illumination has been widely accepted. ${ }^{85-88}$
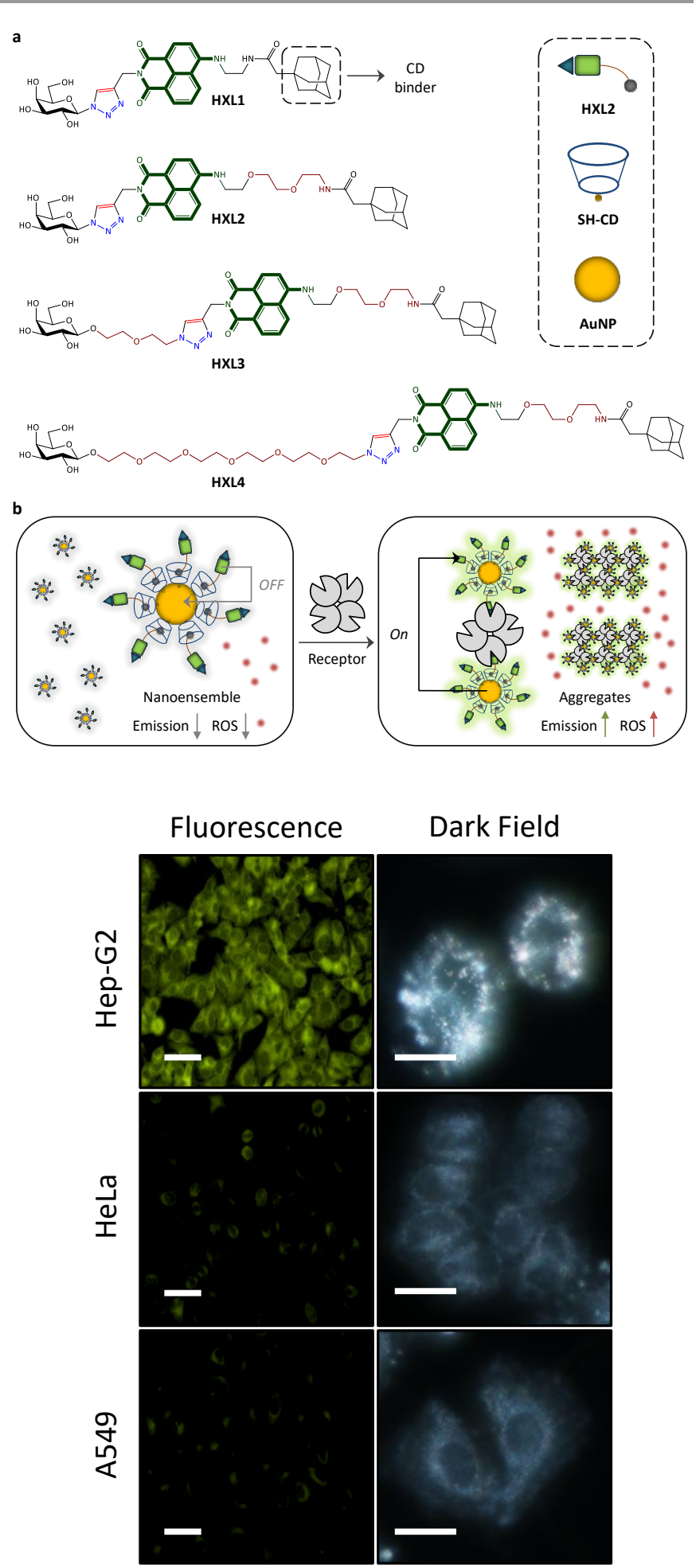

Figure 27 Structures of single probes and illustration of the assembly of the AuNPs with probes and cyclodextrin (SH-CD) along with the aggregation-induced emission enhancement and ROS release (left). The effective intracellular imaging in HepG2 cells (with ASGPr) and unclear images in control cells (right). Reproduced from ref. ${ }^{84}$ with permission from the Royal Society of Chemistry, copyright 2016 
Photo-controlled probes could realize the flexible, controllable sensing of cells by irradiation after cell permeation inside, compared with the former methods. The galactose-based probe containing $\mathrm{NI}$ and spiropyran (photo-switch) moieties have been designed for cell-imaging. ${ }^{89}$ The probe was incubated with HSA (human serum albumin) and the complex formed could permeate into cells. The spiropyran photo-switch was then activated into its green fluorescent merocyanine upon irradiation with UV/Vis light (or turn off because the spiropyran have been transformed into merocyanine). After interacting with the targeted lectin, the emission of this complex would change into red under UV/Vis light, that is because the protein enhanced the emission of merocyanine which is redemitting. This cell imaging strategy could be applied to the selective detection of HepG2 cells with ASGPr-mediated endocytosis (Figure 28).

Previously to these results, a galactose-based probe containing the NI moiety alone for cell-imaging (without protein) has been reported based on similar principles. ${ }^{90}$ The only difference was that after the "turn-off" of green emission of merocyanine, the ion $\mathrm{SO}_{3}{ }^{2-}$ was used to lock the fluorescent "off-on" system, inducing "turn-on", because of the structural change and the blocking of FRET. Then, the green emission again appeared and could no longer "turn-off". This method is actually referred as "traditional" in the above study and the intracellular, photo-controlled imaging have also been realized (also using the galactose moiety targeting HepG2 cells).

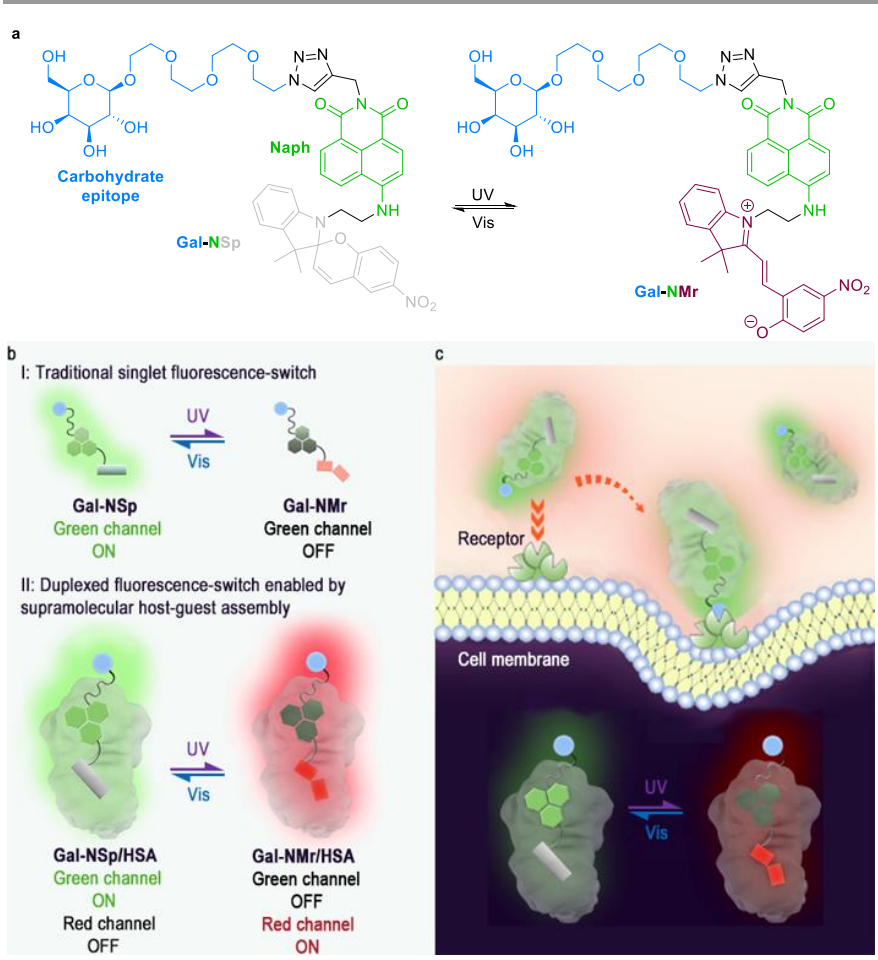

Figure 28 (a) Structures of the single probe and its transformation under irradiation. (b) Schematic representation of the light-induced emission change of both single probe and the HSA/probe complex. (c) Proposed process for endocytosis of the HSA/probe complex. Reproduced from ref. ${ }^{89}$ with permission from the American Chemical Society, copyright 2018.

A selective sensing of hepatocellular $\mathrm{H}_{2} \mathrm{~S}$ was designed using the NI-based galactoside. ${ }^{91} \mathrm{H}_{2} \mathrm{~S}$ is an important gaseous transmitter in many cellular signalling pathways. Its detection in
HepG2 cells was successfully realized in a target-specific imaging of $\mathrm{H}_{2} \mathrm{~S}$ in the liver.

The lysosome inside cells is a very crucial organelle. Galactosylated probes NPGal 1-3 containing the NI fluorophore with various linker arms were synthesized as well as a negative control probe NPP without galactose. ${ }^{92}$ These glyco-probes could sensitively and selectively visualize the lysosome inside HepG2 cells through recognition of the galactoside epitope by the ASGPr and then endocytosis (Figure 29). Interestingly, the probes were $\mathrm{pH}$ sensitive and could turn on their fluorescence in acidic environment such as in lysosomes.
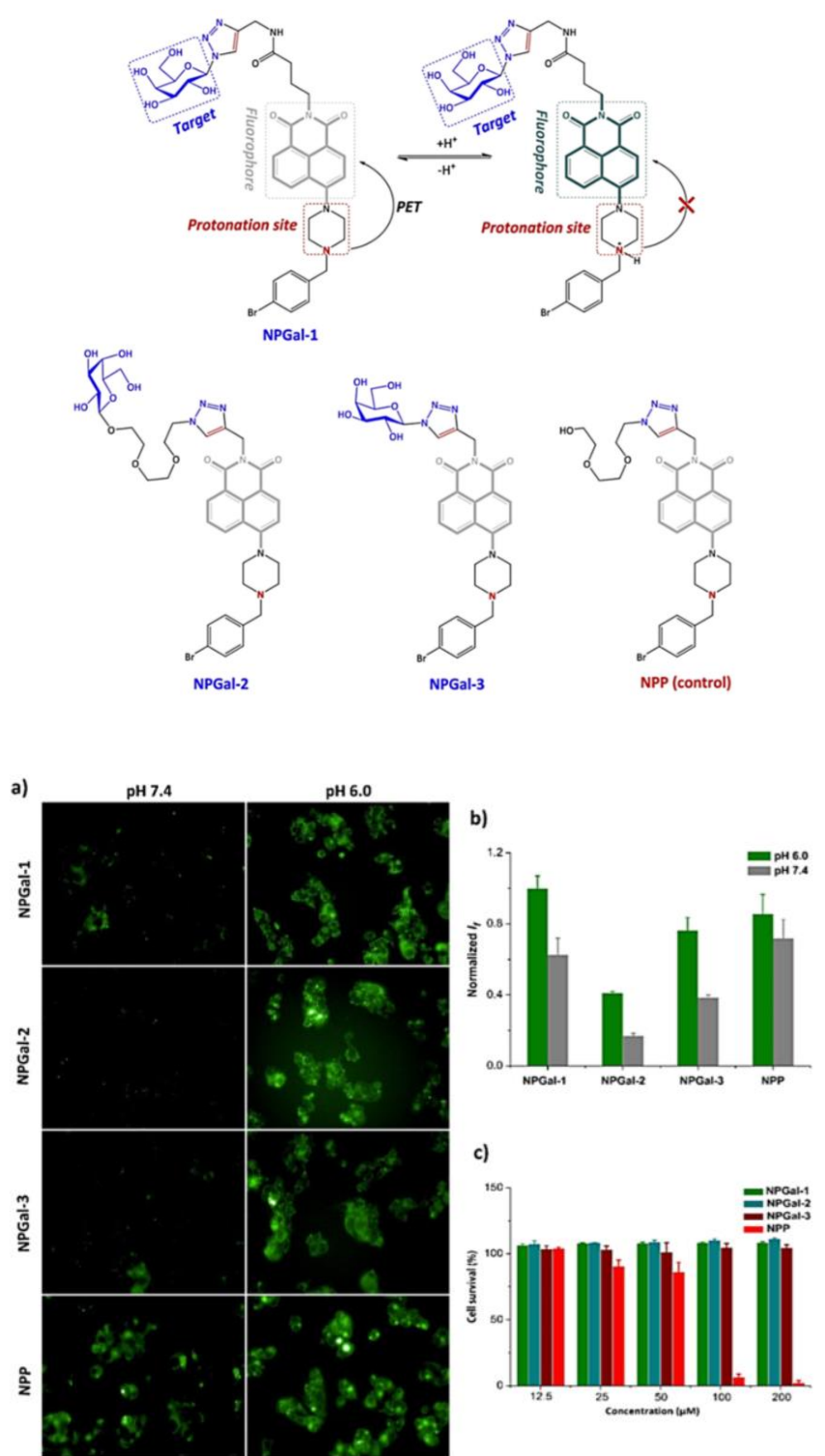

Figure 29 Structures of $\mathrm{pH}$-sensitive $\mathrm{NI}$-based probes. Fluorescence imaging, quantification and cytotoxicity of the probes in lysosome of HepG2 cells $(a, b, c)$. Reproduced from ref. ${ }^{92}$ with permission from the Elsevier B.V., copyright 2016.

A fluorescent bis-galactosylated dithienylethene glycoconjugate incorporating two $\mathrm{NI}$ units and also two pH-sensitive piperazines was synthesized (Figure 30) and its switching properties were studied under UV/Vis light, acid or base (TFA/triethylamine) stimuli. ${ }^{93}$ This glycoconjugate could oper- 
ate as a molecular logic circuit with "AND", "OR", and "NOT" gates.

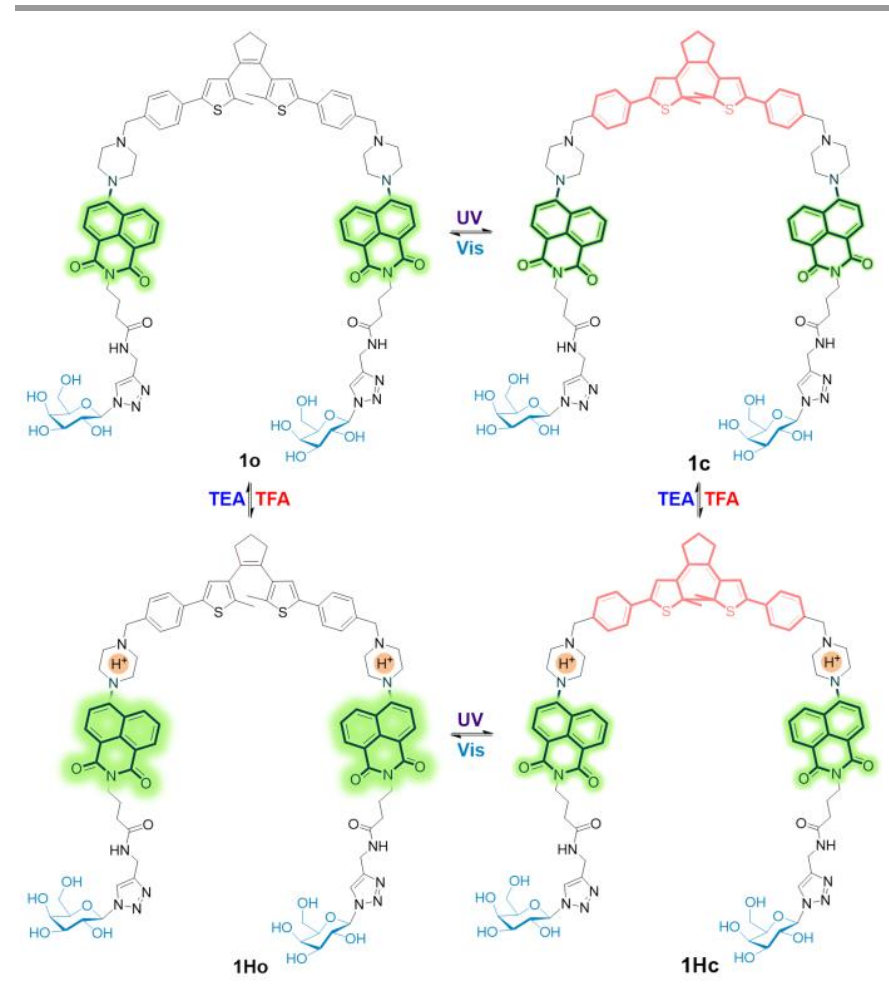

Figure 30 Illustration of the structural transformations in response to UV/Vis and acid/base stimuli. Reproduced from ref. ${ }^{93}$ with permission from the Royal Society of Chemistry, copyright 2017.

The introduction of carbohydrates onto probes influences their properties. The glycosylation of NDI-based probes could enhance their sensitivity and lower their cytotoxicity. ${ }^{94}$ Two glyco-probes with galactose and glucose respectively were used and a non-glycosylated probe was also used as control (Figure 31). The results showed that besides the sensitivity and cytotoxicity, the introduction of carbohydrates could also enhance the working $\mathrm{pH}$ range and targeting ability for specific cells (galactosylated probes for HepG2 cells expressing ASGPr), and could reduce non-specific cellular internalization.

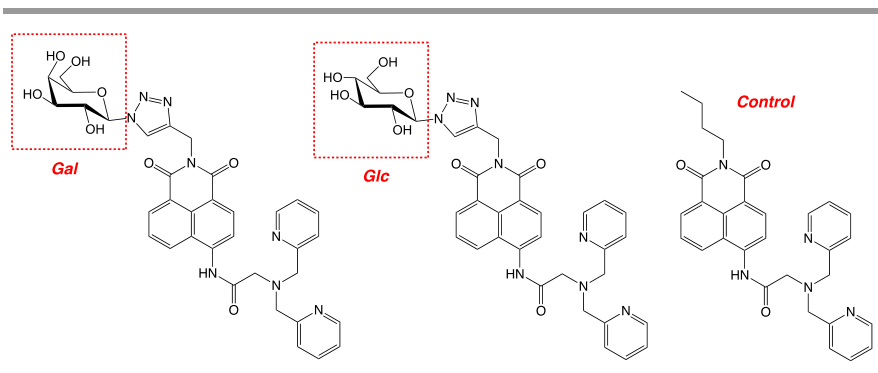

Figure 31 Structure of galactosyl (Gal) probe, glucosyl (Glc) probe and control probe.

\subsection{Boron-dipyrromethene-based glycoconjugates}

Boron-dipyrromethene (4,4-difluoro-4-bora-3a,4a-diaza-sindacene, BODIPY) incorporating a dipyrromethene core in complexation with a boron atom are a class of frequently used fluorophores for the design of probes. ${ }^{95-97}$ This fluorescent probe is convenient owing to both its high photo-chemical stability and its fluorescent quantum yield, its good solubility in most of the organic solvent as well as relative independence of fluorescence on $\mathrm{pH}$ and environmental conditions. The emission wavelength of BODIPYs can range from the visible to the NIR region by fine-tuning their structure. Interestingly, highly fluorescent BODIPY probe display narrow absorption and emission bands with high peak intensities. In addition, it has been demonstrated that the conjugation of a carbohydrate moiety to the BODIPY probe improves significantly the selectivity of the imaging probe toward a specific biological target. A NIR fluorescent BODIPY-type dye was synthesized containing one or two indolylstyryl groups to increase the cellular uptake and biological efficacy of the probe and either a PEG (polyethylene glycol) or carbohydrate moiety to increase the water solubility and cellular targeting of the resulting conjugates (Figure 32). ${ }^{98}$ The absorbance and fluorescence emission spectra have shown any small differences between the glycosylated and the corresponding native BODIPY, which suggested that conjugation of carbohydrates via 1,2,3-triazoles did not alter the spectral properties of the probes. Then, in vitro biological properties were investigated in human carcinoma HepG2 cells. The BODIPY conjugates were non-cytotoxic, both in the presence and in the absence of light, which have highlighted the potential therapeutic application of such molecules. In addition, the mono-substituted indolylstyryl BODIPY have shown higher cellular uptake by the HepG2 cells than the disubstituted indolylstyryl BODIPY. Moreover, BODIPYcarbohydrate conjugates as well as the analogue BODIPY-PEG (containing a PEG group instead of a carbohydrate) were able to accumulate within the cell more efficiently than the native BODIPYs. Finally, fluorescence microscopy has provided useful information about the main sites of subcellular localization of the BODIPYs, which have found preferentially in the cell endoplasmic reticulum (ER).

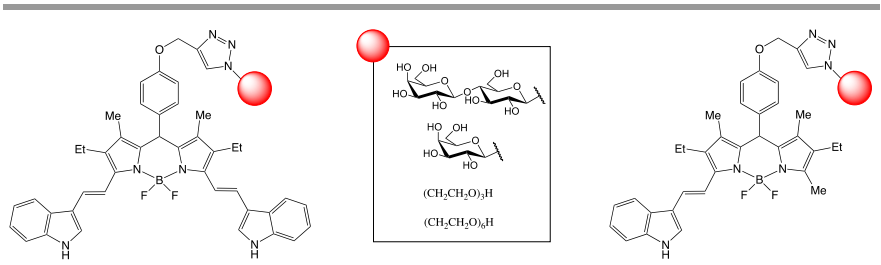

Figure 32 Structures of the glycosylated and PEGylated BODIPYs.

Iminosugars are very attractive carbohydrate analogues mainly used as inhibitors of enzymes (e.g. glycosidases, proteinases, protein kinases and cholinesterases). ${ }^{99-104}$ Since 2010, a large number of multivalent iminosugars glycoclusters based on various platforms have been reported with dramatic increase in the inhibitory properties towards the targeted enzyme. ${ }^{105}$, 106 The synthesis and photophysical properties of BODIPYbased iminosugar clusters ${ }^{107}$ in which the BODIPY has a dual function as core scaffold for the conjugation of carbohydrates but also as a fluorescent probe (Figure 33). The glycoconjugates were designed to incorporate either the classical fluorine-BODIPY or the less common ethynyl-BODIPY since adequate tailoring might induce special sensing properties. The 
absorption spectra of the BODIPY-based glycoclusters have shown characteristic band of the BODIPY subunit and 1,2,3triazole ring. A slight red shift was observed between BODIPYbased glycoclusters and native BODIPY, which might be attributed to the influence of the triazole ring. The emission spectra obtained $\left(\lambda_{\mathrm{ex}}=510 \mathrm{~nm}\right)$ as well as the modest Stokes shift and the short excited state lifetime have suggested a singlet emitting state.

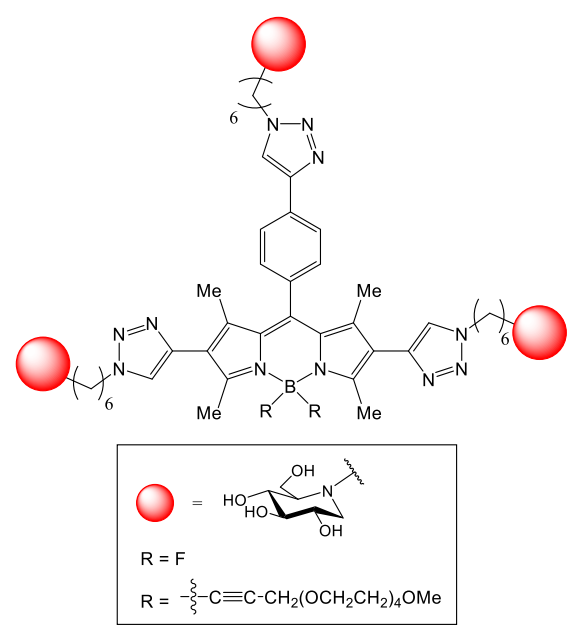

Figure 33 Structures of the functionalized BODIPYs.

Conjugation of glycosides to BODIPY scaffolds can be readily achieved by CuAAC from the propargyl-BODIPY and the 2azidoethyl glycosides (Figure 34). ${ }^{108}$ These water-soluble probes displayed emission from the yellow to blue but were not applied to biological systems.
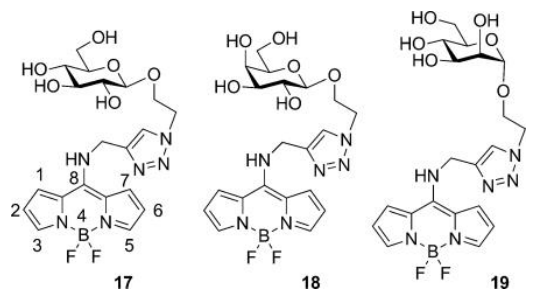

Figure 34 Structures glycosylated BODIPYs. Reproduced from ref. ${ }^{108}$ with permission from the Wiley-VCH, copyright 2014.

A more amphiphilic BODIPY-glucose conjugate was synthesized and the compound could readily undergo self-assembly into liposomes (Figure 35). ${ }^{109}$ The original fluorescence of the BODIPY-glucose amphiphile was shifted from green in solution to red inside the liposome due to higher concentrations of the amphiphile in the liposome quenching the typical green emission and providing red emission by excitation at $587 \mathrm{~nm}$.

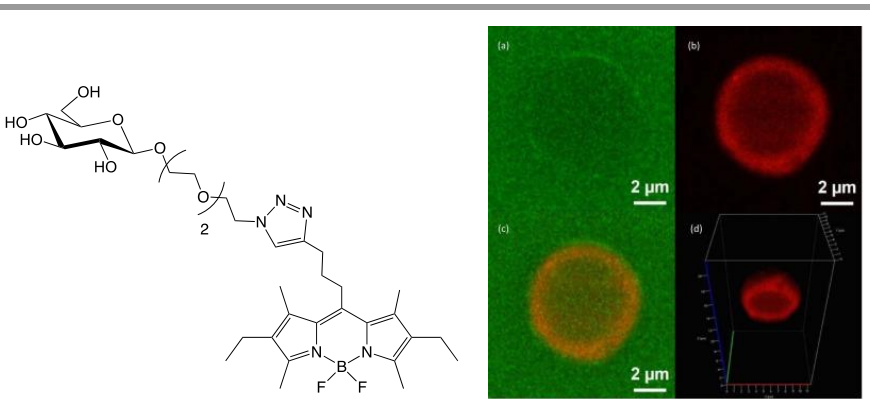

Figure 35 Structure of a glucosylated amphiphilic BODIPY and confocal fluorescence $2 D$ and $3 D$ images of its insertion into liposomes. Reproduced from ref. ${ }^{109}$ with permission from the Elsevier B.V., copyright 2016.

Another amphiphilic BODIPY glycoconjugate (BTM) was designed and synthesized to generate nanomicelles and applied in photodynamic therapy (PDT) of breast cancer (Figure 36). ${ }^{110}$ The BTM glycoconjugate could self-assemble into nanomicelles (BTM-NM) in the presence of tween 80 , then penetrate into cells via a mannose-receptor endocytosis on MDA-MB-231 breast cancer cells. Their accumulation in lysosomes caused their disassembly and an efficient singlet oxygen generation was observed upon light irradiation $(665 \mathrm{~nm})$. Singlet oxygen caused the breakdown of the lysosomal membrane and the BTM released in the cytoplasm resulted in the killing of the cancer cell. A negative control with glucosylated nanomicelles proved the selective targeting of the cancer cells.

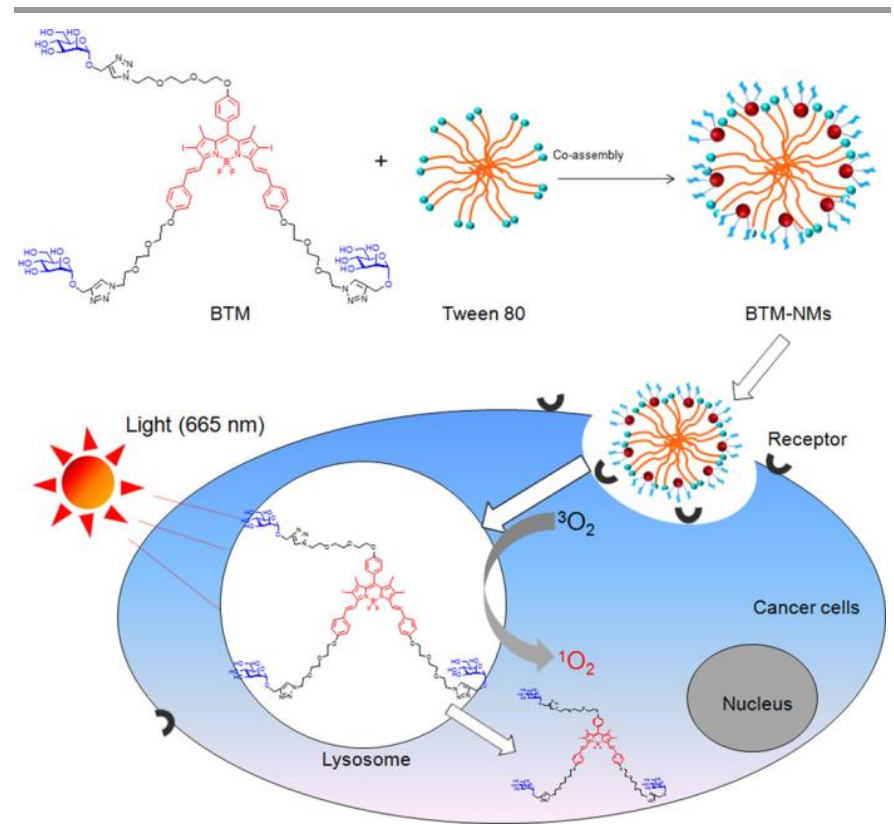

Figure 36 BODIPY-mannoside conjugate and their self-assembly into nanomicelles for further photodynamic therapy (PDT) applications on breast cancer cells. Reproduced from ref. ${ }^{110}$ with permission from the Wiley-VCH, copyright 2017.

Two BODIPY moieties were conjugated onto a Gal-??-(1,4)-GIC disaccharide scaffold at the 2 '-position and reducing end (Figure 37). ${ }^{111}$ The BODIPY units had different absorption/emission properties and the luminescence spectrum displayed an intense absorption at $770 \mathrm{~nm}$ for the lower energy BODIPY. A very efficient energy transfer could be observed from the BODIPY with highest energy to the one with lowest, and the 
emission of the glycoconjugate was in the near infrared window in agreement with potential biological applications.

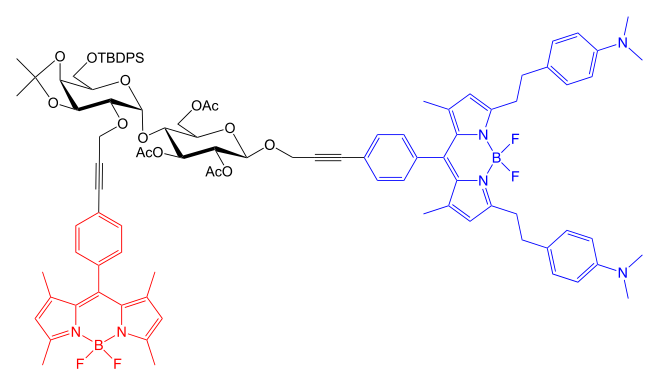

Figure 37 Conjugation of two BODIPY moieties into a bichromophoric system based on a disaccharide scaffold.

BODIPY was used as a core scaffold for the design of glycoconjugates bearing from one to three galactoside moieties (Figure 38). ${ }^{112}$ Azide-alkyne "click" conjugations were performed at the meso-position and through propargyl residues at the boron atom. Cell assays using HepG2 cells (liver cancer) indicated that the BODIPY conjugates are non-cytotoxic and their cellular uptake increased with the number of glucose residues.
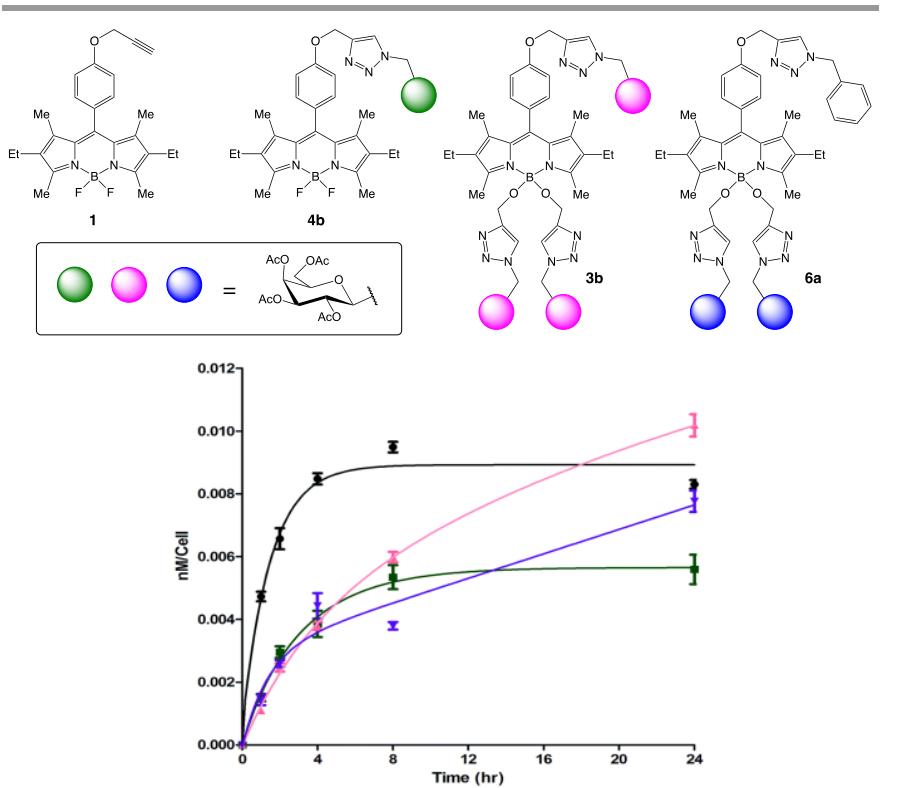

Figure 38 Structures of the galactosylated BODIPY glycoconjugates and their time-dependent uptake of BODIPYs 1 (black), 3b (pink), 4b (green) and $6 \mathbf{a}$ (purple) at $10 \mu \mathrm{M}$ by HepG 2 cells. Reproduced from ref. ${ }^{112}$ with permission from the Royal Society of Chemistry, copyright 2018.

\subsection{Fluorescein-based glycoconjugates}

Fluorescein, a low-toxic fluorophore typically displaying a bright green fluorescence emission, is among the most extensively used tracing reagent often conjugated with biomolecules for bio-imaging and biosensors. ${ }^{113-120}$ Fluorescein and its derivatives are one of the most common fluorescent dyes found in the literature, mainly due to their excellent fluorescence and photo-physical properties such as its high intensity emission peaks, high molar absorption coefficients, appropriate fluorescence quantum yield and its stability under irradiation conditions. The photo-physical and optical properties of fluorescein in solution display a strong dependence of the molecular structure of the dyes, since substitution of the probe may, in some instance, modify both electronic absorption and maximum emission due to the change in the electronic environment of the molecular structure of the probe. ${ }^{121,122}$ Typical "off-on" type fluorescein-based probes can be easily designed using the spirolactone-ring opening mechanism. ${ }^{123}$ A very efficient route for the synthesis of fluorescein-based $N$ glycosamine derivatives has been designed ${ }^{124}$ through conjugation of 1,6-benzylidene-protected glucopyranose with a fluorescein bis-aniline (Figure 39). Low molecular-weight organogelators (LMOGs) are gelator molecules self-assembling into supramolecular structures via weak noncovalent interactions. The gels derived from these biomolecules were applied in medicinal chemistry and material science based on the biocompatibility of carbohydrates. The gelation properties of bis$N$-glycosylated fluorescein-based glycoconjugates were influenced predominantly by the protecting groups and the substituents present on the fluorescein aromatic core. Also, the etherified fluorescein-based probes have shown better gelation ability than the corresponding esters. The cell imaging study of these molecules revealed the exclusive localisation of fluorescence in the cytoplasm, but not in the membrane of cancer cells, which demonstrated the anticancer potential of this class of fluorescein-based glycoconjugate.

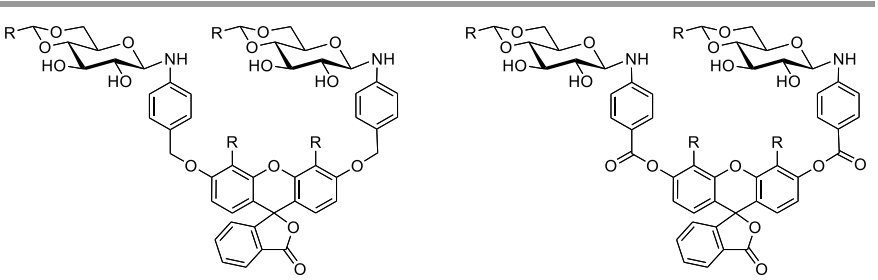

Figure 39 Structure of the bis- $N$-glycosylated fluorescein-based glycoconjugates.

The same group also described a novel class of fluoresceinbased $\mathrm{O}$-glycosides by means of direct glycosylation of a fluorescein bis-benzylic alcohol (Figure 40). ${ }^{125}$ The antioxidant properties of the resulting fluorescein-based glycoconjugates were assessed for their capacity to prevent the formation of 1,1-diphenyl-2-picrylhydrazine (DPPH) and have shown weak to moderate activities compared to $\alpha$-tocopherol used as the reference. In addition, preliminary biological evaluations have highlighted the antibacterial and antifungal activities of these fluorescein-based $\boldsymbol{O}$-glycosides.

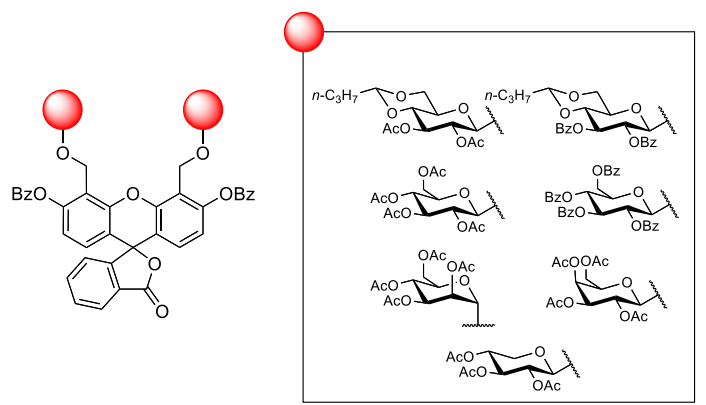

Figure $\mathbf{4 0}$ Structures of the bis-O-glycosylated fluorescein-based glycoconjugates.

In order to design more efficient drug candidates, the same group has proposed to conjugate fluorescein derivatives to a $C$-glycosyl residue leading to the fluorescein-based $C$ - 
glycosides through aldol condensation/dehydration (Figure 41). ${ }^{126}$ Several partially protected and fully protected monosaccharides have been conjugated and the resulting fluorescein-based $C$-glycosides have shown interesting antioxidant activities.

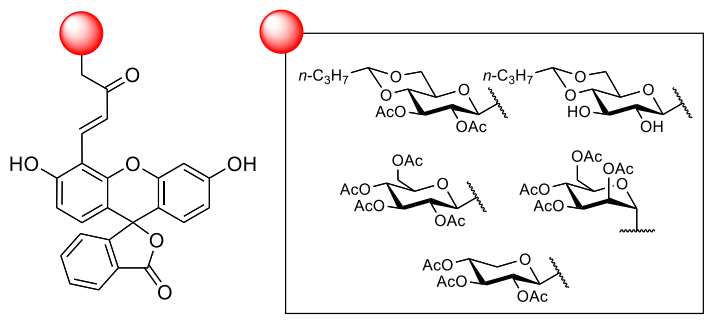

Figure $\mathbf{4 1}$ Structure of the fluorescein-based $C$-glycosides.

The synthesis of fluorescein-based quinoline glycoconjugates was described using a one-pot and three-component procedure starting from a fluorescein aldehyde, anilines and a propargyl glycoside under $\mathrm{Cu}(\mathrm{I})$-catalysis (Figure 42). ${ }^{127}$ These quinoline glycoconjugates displayed moderate antioxidant properties, while the substituents of the aniline have a significant influence on the activity.

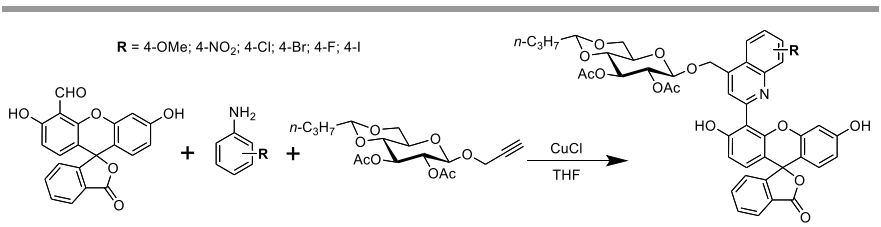

Figure 42 Synthesis of fluorescein-based quinoline glycoconjugates.

The Mannich reaction involving fluorescein aldehyde, anilines and protected $C$-glycosyl-acetone in the presence of a catalytic amount of potassium carbonate has been performed for the synthesis of fluorescein-based $C$-glycoside in good yield (Figure 43). ${ }^{128}$ The gelation properties were studied by several techniques including HRTEM (High-Resolution Transmission Electron Microscopy), DSC (Differential Scanning Calorimetry) and powder XRD (X-Ray Diffraction). These compounds were found to be appropriate organogelators, although the preliminary biological evaluation of these fluorescein-based $C$-glycoside has shown moderate antioxidant activities.

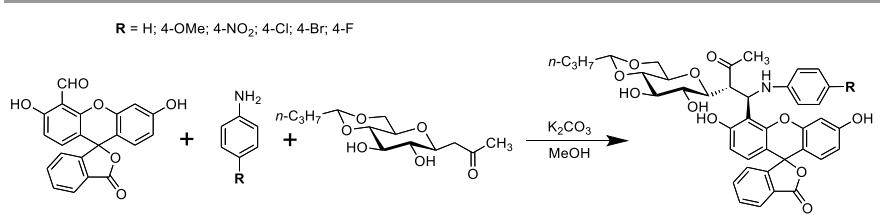

Figure $\mathbf{4 3}$ Synthesis of fluorescein-based C-glycoside.

\subsection{Rhodamine-based glycoconjugates}

Rhodamine, which is structurally similar to fluorescein, is another class of biocompatible dyes for biological imaging and probes design. ${ }^{129-132}$ However, the emission wavelength of rhodamine (> $580 \mathrm{~nm}$ ) is typically longer than fluorescein. Substitution of the rhodamine core can strongly influence the absorption and emission properties of the fluorescent probe. The open and close forms of the spirolactam-ring of rhodamine can also be reversed upon complexation with analytes (e.g. cations) or change under external stimuli (e.g. decrease of
$\mathrm{pH}) .{ }^{132-136}$ Therefore, rhodamine-based probe shows excellent absorbance and fluorescence intensity changes upon interaction with some specific metal ions.

Considering the versatility and efficiency of the synthesis of fluorescein glycoconjugates presented previously, the preparation of rhodamine-based $\mathrm{N}$-glycosylamine derivatives was readily accomplished from partially protected glycosides and amino-functionalized rhodamine-based derivatives to give the corresponding glycoconjugates in good yields (Figure 44). ${ }^{137}$ These compounds act as efficient organogelators, while further analyses have shown various modes of aggregation and stability of gels, which seem largely influenced by the protecting groups on the carbohydrate moiety as well as the substituents on the rhodamine moiety.

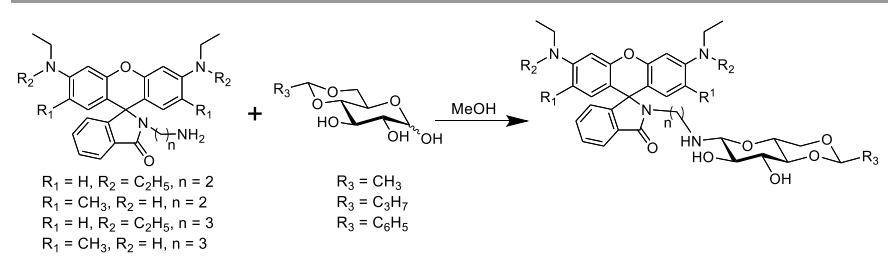

Figure $\mathbf{4 4}$ Synthesis of rhodamine-based $\mathrm{N}$-glycosylamine glycoconjugates.

Divalent mercury ion $\left(\mathrm{Hg}^{2+}\right)$ is extremely toxic to the environment. Consequently, a wide range of ratiometric probes have been developed in recent years for its detection. Comparing to the conventional fluorimetric probes, the advantage of a ratiometric probe is their shiftable fluorescence emission wavelengths, which significantly improve the specificity of the probe. For this purpose, glycosyl rhodamine derivatives have been designed as novel fluorescent probe for $\mathrm{Hg}^{2+} .{ }^{138}$ Conjugation of 1-azido-glycosides with propargyl rhodamine through CuAAC afforded the desired rhodamine-based glycoconjugates (Figure 45). Interestingly, this probe could detect selectively $\mathrm{Hg}^{2+}$ in $\mathrm{H}_{2} \mathrm{O} / \mathrm{MeCN}(4: 1, v / v)$ through a $1: 1$ probe-ion complex and selectively in the presence of a range of other metal ions $\left(\mathrm{Cu}^{2+}\right.$, $\mathrm{Ni}^{2+}, \mathrm{Co}^{2+}, \mathrm{Zn}^{2+}, \mathrm{Pd}^{2+}, \mathrm{Ag}^{+}, \mathrm{Mn}^{2+}, \mathrm{Cd}^{2+}, \mathrm{Fe}^{3+}, \mathrm{Cr}^{2+}$ ). UV-vis and fluorescence spectroscopic analyses have shown that upon formation of a probe-ion complex, the quenching of the blue fluorescence of glycosyl rhodamine was correlated with the emergence of a red fluorescence emission corresponding to the lactam ring-open form of the rhodamine moiety. A plausible mechanism was proposed, claiming that upon addition of $\mathrm{Hg}^{2+}$ solution, a nitrogen atom of the triazole, oxygen atom of the alkyl chain and the carbonyl group of the rhodamine were able to chelate the mercury ion, resulting in opening of the spirolactam ring of rhodamine, allowing an intramolecular FRET with the galactoside and leading to a red fluorescence emission (Figure 45).

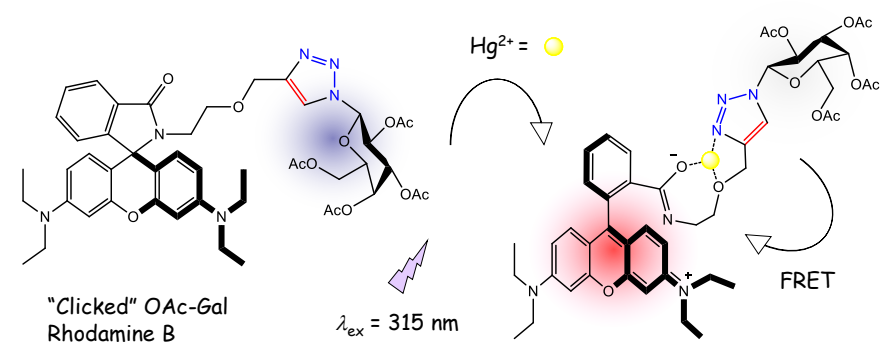


Figure 45 Synthesis of the rhodamine-based glycoconjugates and proposed mechanism for the ring-opening of the spiro-lactam upon complexation with $\mathrm{Hg}^{2+}$. Reproduced from ref. ${ }^{138}$ with permission from the Elsevier B.V., copyright 2014.

Similarly, the synthesis of a bis-galactosylated rhodaminebased probe was reported and its application as water-soluble probe for imaging $\mathrm{Hg}^{2+}$ selectively in heptoma cells over other cancer cells. ${ }^{139}$ Conjugation through CuAAC was accomplished from a bis-propargyl rhodamine and 1-azido-galactoside. The proposed mechanism of the fluorescence of the resulting bisgalactosylated rhodamine-based glycoconjugate in the presence of $\mathrm{Hg}^{2+}$ is relatively similar to that described above. The cellular imaging ability of the probe was studied in HepG2 cells for which the probe could detect intracellular $\mathrm{Hg}^{2+}$. Reduction or inhibition of the ASGPr expressed selectively on the membrane of HepG2 cells led to drastic decrease of the imaging ability of the probe, which indicates that the probe's endocytosis was mediated by the ASGPr (Figure 46). The free galactose was also used to compete the binding site of the ASGPr with the probe, leading to inhibition of fluorescence.
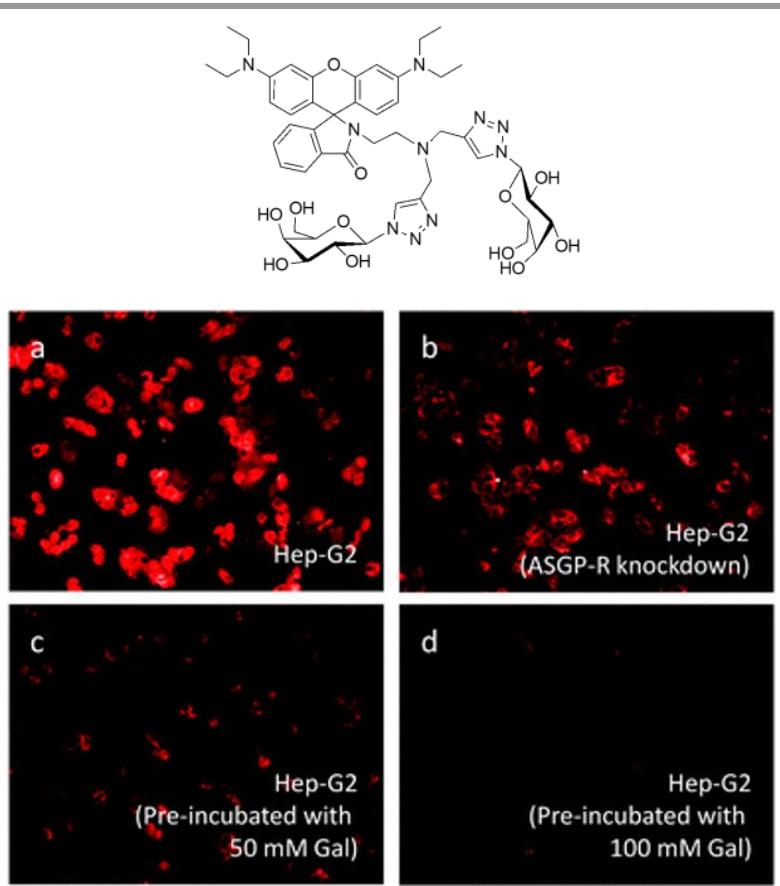

Figure 46 Structure of the bis-galactosylated rhodamine-based glycoconjugate. The imaging of $\mathrm{Hg}^{2+}$ in HepG2 cancer cells with (a) or without (b) ASGPr and in HepG2 with free galactose (c,d). Reproduced from ref. ${ }^{139}$ with permission from the Royal Society of Chemistry, copyright 2014.

Increasing the number of carbohydrate residues should improve the water solubility of such ratiometric fluorescent probes. Therefore, the trivalent glycosyl-rhodamine probe was synthesized through CuAAC click reaction from the corresponding 1-azido-glycoside and the tris-propargylated rhodamine (Figure 47). ${ }^{140}$ The resulting trivalent glycoconjugate has shown a good water-solubility as well as a great selectivity toward $\mathrm{Hg}^{2+}$. The proposed mechanism involves a 1:1 stoichiometric probe-ion complex, in which one nitrogen atom of each 1,2,3-triazole rings and the carbonyl group of the rhodamine participated in the binding of the glycoconjugate probe to $\mathrm{Hg}^{2+}$, leading to the ring-opening of the spirolactam ring of rhodamine and a characteristic fluorescent wavelength emission.

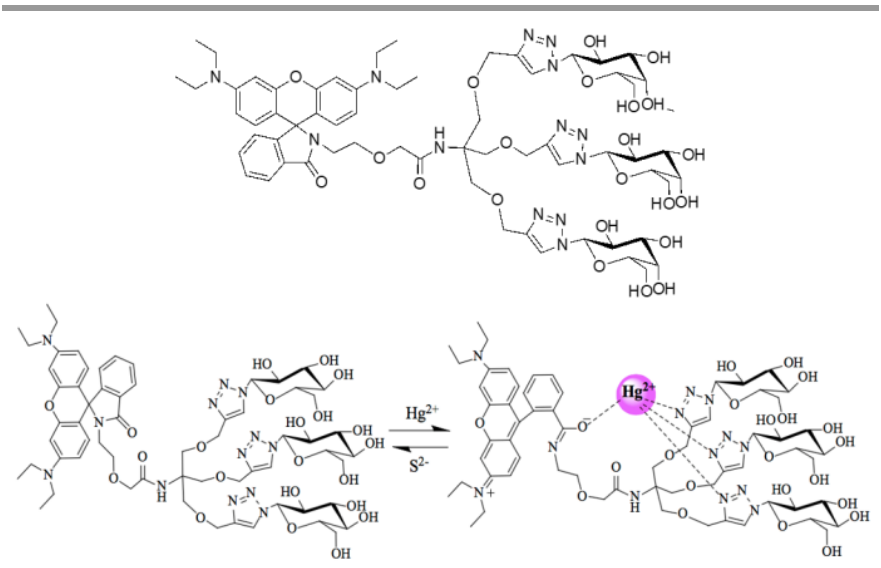

Figure 47 Structure of the tris-galactosylated rhodamine-based glycoconjugates and proposed "off-on" fluorescent mechanism. Reproduced from ref. ${ }^{140}$ with permission from the Elsevier B.V., copyright 2016.

The self-assembled particles based on glycosylated rhodamine and poly( $p$-phenylethynylenes) (PPEs) were investigated for the fluorogenic recognition of lectins. ${ }^{141}$ Two glycoconjugate probes based on the positively charged rhodamine $B$ and two probes using the negatively charged fluorescein were synthesized and nanoparticles with PPEs were then prepared (Figure 48). The introduction of lectins would turn on the fluorescence of glycosylated probe by decomplexation from the PPEs scaffold. The selective detection of PNA, ConA and soybean agglutinin (SBA) by the galactosylated, glucosylated and GalNAcbased probes was realized, respectively.

a
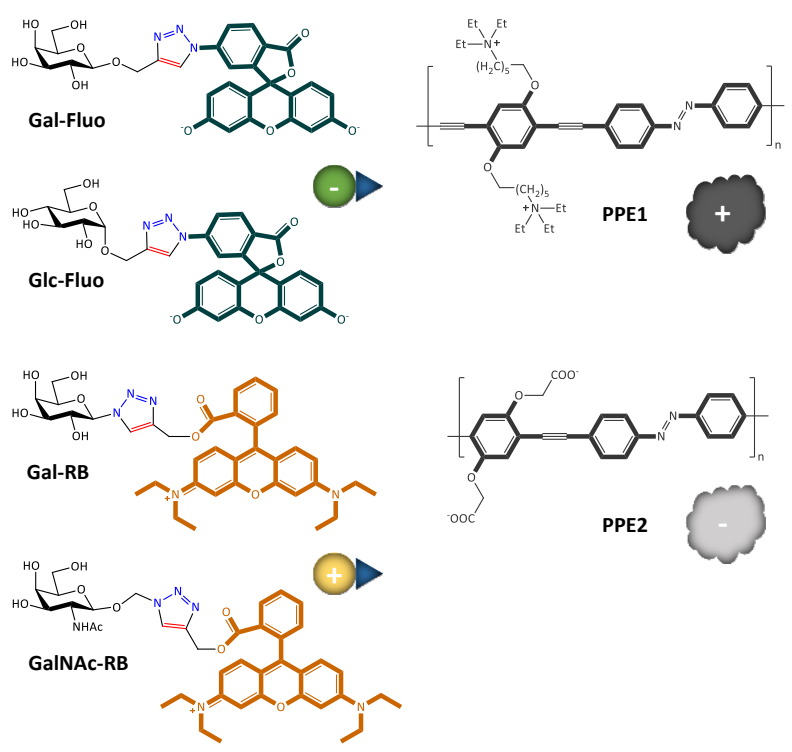
b

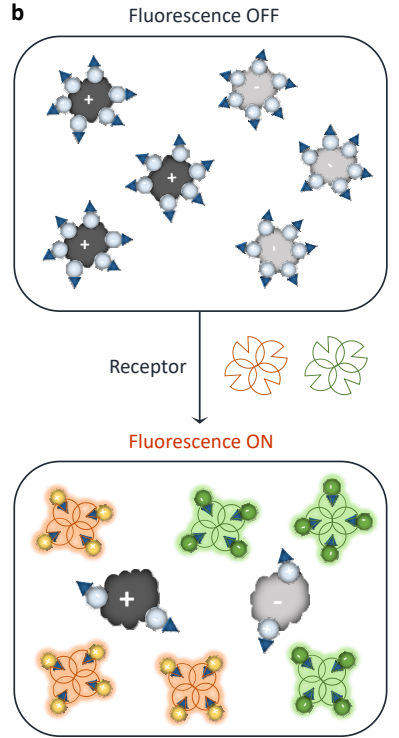

Figure 48 (a) Structures of four probes based on fluorescein or rhodamine B with galactose or $\mathrm{N}$-acetyl-galactosamine (GaINAc), and of poly( $p$-phenylethynylenes) (PPEs) (b) Proposed explanation of the recognition between the nanoparticles and lectins. Reproduced from ref. ${ }^{141}$ with permission from the American Chemical Society, copyright 2016.

Based on similar principles, another self-assembly with poly(3hexylthiophene-2,5-diyl) (P3HT) and glycosylated rhodaminebased probes was reported. ${ }^{142}$ The mannosylated probes could recognize ConA, and PNA required galactoside residues. It was also proved that the mannose-containing probe could respond to the mannose-selective Lens culinaris agglutinin (LCA).

The self-assembled structure of a rhodamine-based glycopolymer with helical protein-microtubule-like structure was also reported. ${ }^{143}$ This self-assembly was formed by carbohydrate-lectin (SBA) interactions and additional $\pi-\pi$ stacking from rhodamine (Figure 49). These protein microtubes could enhance the immune response, demonstrating their potential for biological applications. Similar data was reported with monosaccharides such as glucose, mannose, and galactose with selfassembling properties through rhodamine B dimerization. ${ }^{144}$

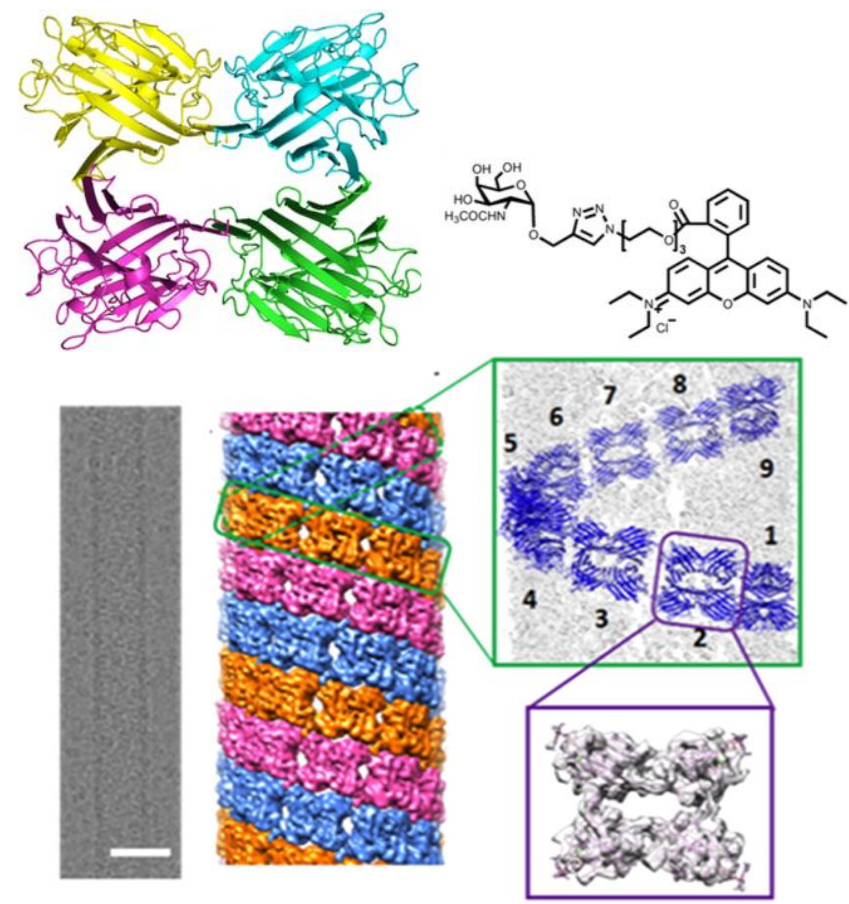

Figure 49 Illustration of the self-assembled helical structures formed by carbohydrate-lectin interactions. Lectin and rhodamine-based glycopolymer and its three-dimensional organization. Reproduced from ref. ${ }^{143}$ with permission from the American Chemical Society, copyright 2016.

NIR fluorescent glucose probes, with low molecular weight and high brightness are able to monitor the cellular glucose uptake. ${ }^{145}$ Interesting applications of these probes might be the detection of cancer, since cancer cells consume more glucose (i.e. produce more energy) than normal cell. Two glucose fluorescent probes have been designed, each of them incorporates a silicon atom instead of the oxygen atom usually found with rhodamine derivatives. This substitution results in the shifts of the emission wavelength to a convenient NIR range. It has been demonstrated that physicochemical properties of the rhodamine core could modulate the cellular uptake of the probes. For this purpose, two glucose-rhodamine glycoconjugates, namely Glc-SiR- $\mathrm{CO}_{2} \mathrm{H}$ and Glc-SiR-Me, bearing distinct net charges ( 0 and +1 , respectively) have been synthesized (Figure 50). While both probes have shown similar excitation/emission wavelength as well as identical extinction coefficient and quantum yield, Glc-SiR- $\mathrm{CO}_{2} \mathrm{H}$ have a better cellular uptake than Glc-SiR-Me, which confirms that net charge of probes have an influence on the cellular uptake behaviour of the glycoconjugate. The mitochondrial respiration viability test, ${ }^{146}$ competition assay with $\mathrm{D} / \mathrm{L}$-glucose as well as difference of cellular uptake between normal and cancer cells have demonstrated that Glc-SiR- $\mathrm{CO}_{2} \mathrm{H}$ displayed low cytotoxicity and have confirmed that the cellular uptake of the glycoconjugate was the result of GLUT-dependent (glucose-transporters) pathways followed by a metabolic phosphorylation. In addition, monitoring the difference of cellular glucose uptake upon treatment with anticancer molecules suggest a useful application of Glc-SiR- $\mathrm{CO}_{2} \mathrm{H}$ for imaging-based diagnostic of cancer (Figure 50). 

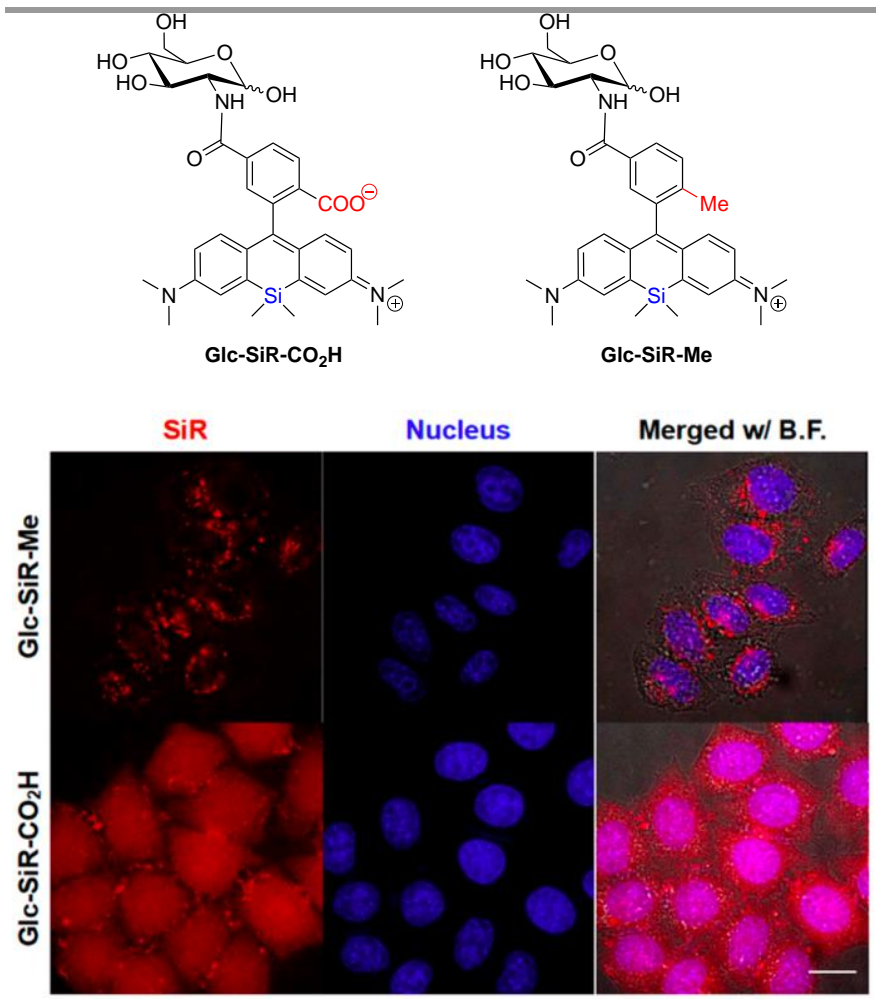

Figure $\mathbf{5 0}$ Chemical structures of Glc-SiR- $\mathrm{CO}_{2} \mathrm{H}$ and Glc-SiR-Me probes and the imaging indicating cellular uptake of Glc-SiR- $\mathrm{CO}_{2} \mathrm{H}$ in HeLa cells. Reproduced from ref. ${ }^{145}$ with permission from the American Chemical Society, copyright 2018.

\subsection{Dicyanomethylene $\mathbf{4} \boldsymbol{H}$-pyran-based glycoconjugates}

Dicyanomethylene $4 H$-pyran (DCM) is a widely used organic dopant for OLEDs (organic light-emitting diodes). ${ }^{147,148}$ Based on its high biocompatibility, fluorescence brightness, longwavelength emission, excellent photostability and high fluorescent yield, DCM-based fluorescent probes for biomedical applications have been extensively developed in recent years. ${ }^{140,149-153}$ The fluorescence emission color of DCM is red, and the DCM-based glycoconjugates are mainly prepared by CuAAC conjugation using a reactive functionality on the periphery of the DCM scaffold.

Our group has reported several studies about DCM-based glycoconjugates and the design to conjugate them through non-covalent interactions onto specific 2D materials for biomedical applications. Carbohydrate-based probes containing fluorescent moieties such as DCM or aminocoumarin (AC) were adsorbed onto the surface of graphene oxide (GO) through ?-? interactions leading to a single-excitation, dualemission graphene composite. ${ }^{154}$ Applications for the detection of multivalent interactions with lectins was demonstrated using mannosylated or galactosylated probes (Figure 51). The initial fluorescence emission of the probes was quenched a lot after adsorption onto GO. The carbohydrate epitopes would then bind to their lectin, triggering the release from the 2D material surface and inducing a fluorescence recovery with a single excitation. This method allowed the simultaneous detection of two lectins (PNA and ConA) in a mixture.

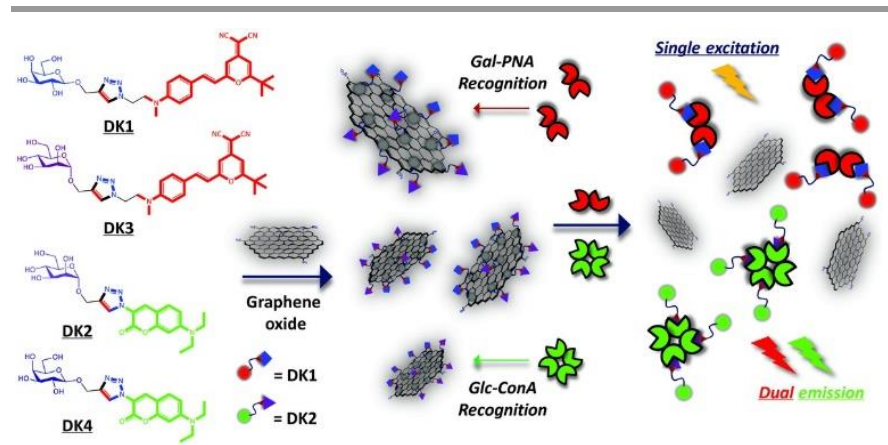

Figure 51 Structures of the glycosylated DCM-based and aminocoumarin-based probes used for complexation with GO and schematic representation of the simultaneous detection of two lectins. Reproduced from ref. ${ }^{154}$ with permission from the Wiley-VCH, copyright 2015.

The DCM-based fluorescent galactosides or mannosides were synthesized and used for the ratiometric detection of $\beta$ amyloid $(A \beta)$ and discrimination from lectins by a selfassembled supramolecular AIE-glyconanoparticle (AIE-GNP) (Figure 52). ${ }^{155}$ The fluorescent AIE-GNP probes consist of a silole-diyne with AIE property and a DCM-based glycoconjugate. The AIE-GNP could sensitively detect monomeric $A \beta$ peptides as well as their fibrils in a ratiometric system. Meanwhile, lectins selectively recognized the glyco-probes, inducing a different ratiometric response.

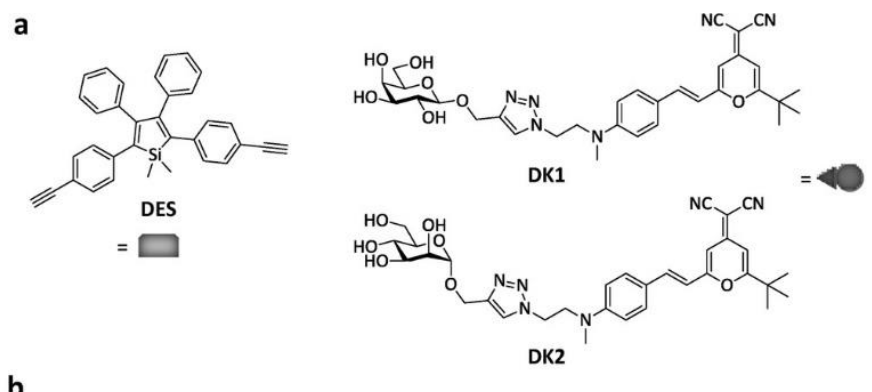

b

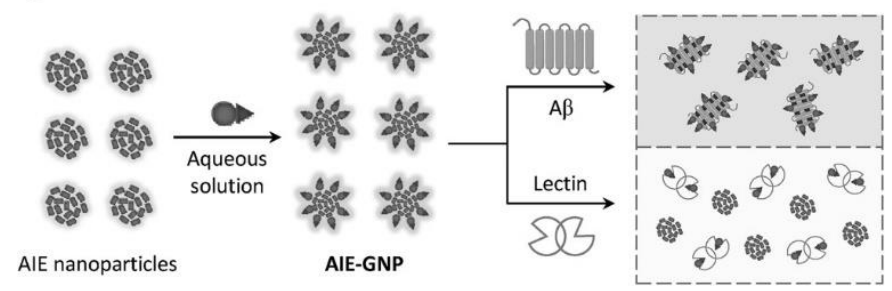

Figure 52 (a) Structures of the DES and the DCM-based fluorescent galactosides or mannosides. (b) Supramolecular assembly of DES with glyco-probes to produce the AIE-GNP and its application for the ratiometric detection of $A \beta$ and discrimination between $A \beta$ and lectins. Reproduced from ref. ${ }^{155}$ with permission from the Wiley-VCH, copyright 2016.

Apart from simply detecting and recognizing lectins, the use of glycosylated DCM-based probes could also expand to biomedical areas. Based also on GO, the galactosylated DCM-based probe (Figure 53) was used to design a dual cell imaging and drug delivery system. ${ }^{156}$ The galactoside is used for its specific multivalent recognition by the ASGPr on HepG2 cells and led to their imaging by the DCM-based probe. Moreover, an effective drug delivery system could be established when camptothecin (CPT) was co-functionalized on GO with the DCM-based probe. Meanwhile, if CPT was only combined with the DCM-based 
probe without GO, only weak monovalent interactions and little amount of CPT was transferred into cells.

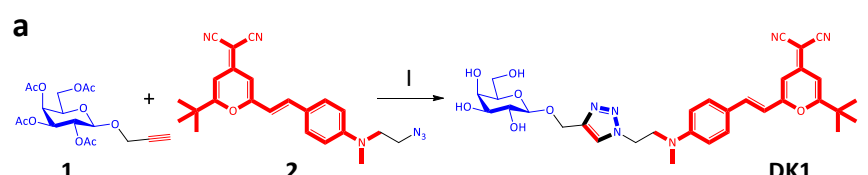

b

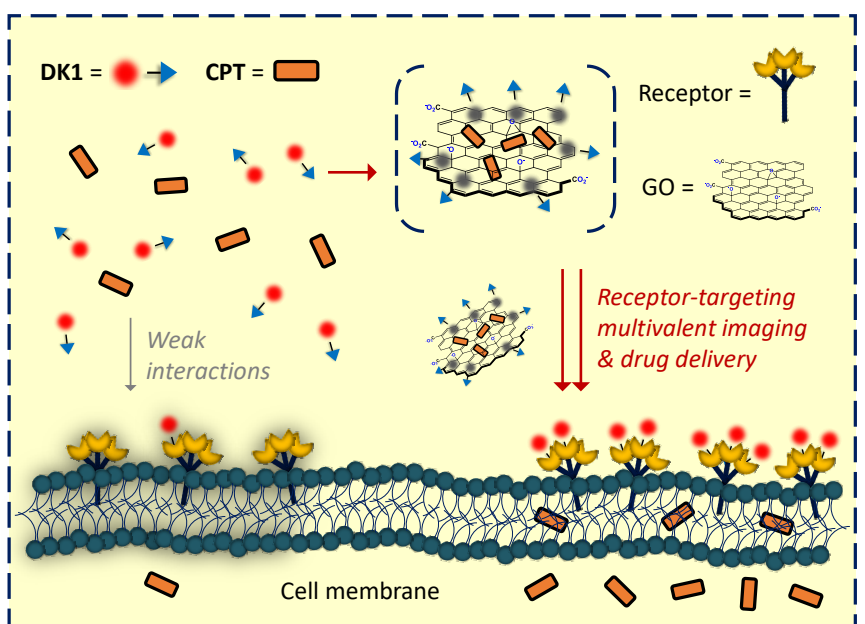

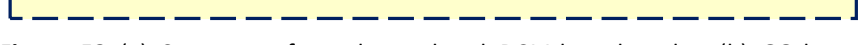

Figure 53 (a) Structure of a galactosylated DCM-based probe. (b) GO-based materials with CPT drug to construct into a receptor-targeting based cell imaging agent and drug delivery system. Reproduced from ref. ${ }^{156}$ with permission from the Royal Society of Chemistry, copyright 2015.

Glycosylated DCM-based probes were conjugated with lamellar 2D MoS 2 leading to simple 2D glycosheets. ${ }^{157}$ These complexes were designed for targeted cell imaging and intracellular production of ROS, since lamellar materials like $\mathrm{MoS}_{2}$ could release ROS or radicals under light-irradiation to realize PDT, thus having the anticancer potential (Figure 54). Among three probes, the DK1 with single galactose decorated showed the best affinity towards ASGPr expressed on HepG2 cells due to the specific recognition. After incubating with HepG2 cells, galactose linked probe DK1 alone did not exhibit a remarkable imaging effect (low-affinity monovalent interactions with ASGPr), but the glycosheets provided much stronger imaging ability for Hep-G2 through a multivalent clustering of ASGPr on the HepG2 cell surface. Then selectivity was assessed by incubating the glycosheets with Hep-G2 and control cells and light irradiation was induced to release ROS inside cells. The experiment of glycosheet containing galactose-based probes with the cells significantly enhanced the ROS concentration in Hep-G2, but not in control cells, which have no ASGPr expression.

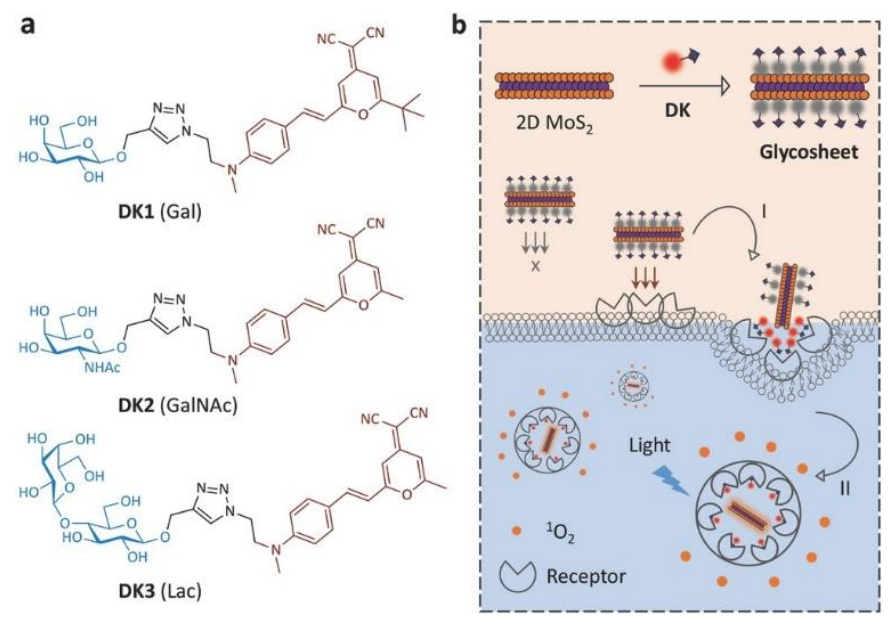

Figure $\mathbf{5 4}$ (a) Structures of some glycosylated DCM-based probes (Gal = galactose; GalNAc $=N$-acetyl-galactosamine; Lac $=$ lactose). (b) supramolecular selfassembly between 2D MoS 2 and glycosylated DCM-based probes to form glycosheets and their targeted production of singlet oxygen by the control of (I) ASGPr-mediated endocytosis and (II) light irradiation into cells. Reproduced from ref. ${ }^{157}$ with permission from the Wiley-VCH, copyright 2016.

Apart from 2D materials, DCM-based self-assembled glyconanoparticles as another functional materials have also been studied in these biomedical areas. A DCM-based supramolecular assembly with polymer dots (poly(9,9-dioctylfluorenyl-2,7diyl (PFO) and poly(styrene-co-maleic anhydride) (PMSA)) was reported for the detection of lectins as well as the potential use in delivery of imaging agents (Figure 55). ${ }^{158}$ The specific binding to lectins leading to fluorescence changes was similarly tested with ConA and PNA. Similar imaging results were obtained showing HepG2 cells could absorb the galactosylated probe to deliver the imaging agent intracellularly while other cells like HeLa cannot. And, the supramolecular ensemble with galactose could be recognized by ASGPr, while the one with mannose cannot. 
a
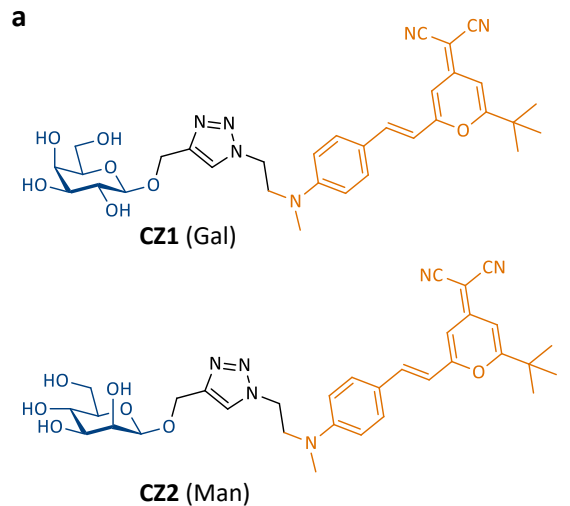

CZ2 (Man)<smiles>CCCCCC1(CCCCC)c2cc(C)ccc2-c2ccc(C(F)(F)F)cc21</smiles>

PFO

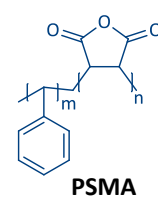

PSMA

b

PFO based polymer dot
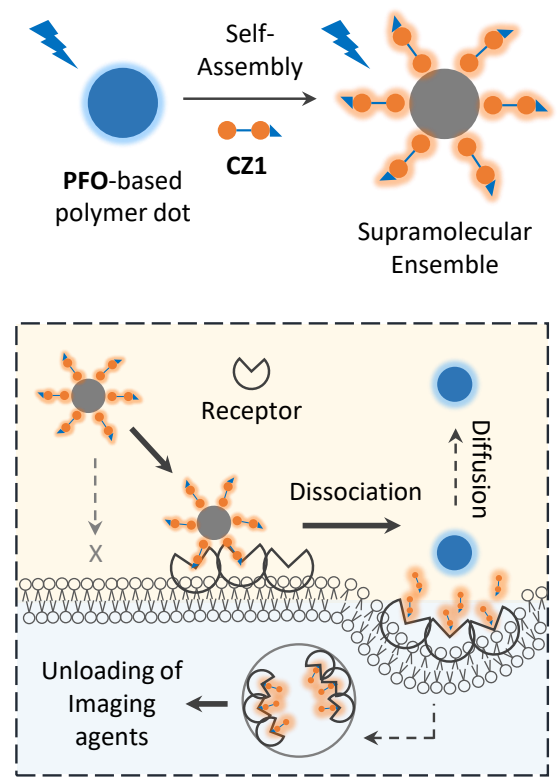

Figure 55 (a) Structures of fluorescent glycosylated DCM-based probes and polymer dots which is formed by mixing with PFO and PMSA. (b) Process for the self-assembly of the supramolecular polymer system and the intracellular ASGPrcontrolled dissociation and unloading of the imaging agent. Reproduced from ref. 158 with permission from the American Chemical Society, copyright 2017.

Self-assembled glyco-dots made from PDI-based, galactosylated or mannosylated glycoclusters and a DCM non-glycosylated probe could be used for selective and targeted cancer cell imaging in HepG2 cells or MDA-MB-231 cells as discussed before. ${ }^{64}$ Moreover, the self-assembly of DCM-based glycoconjugates with $\mathrm{P} 3 \mathrm{HT}$ polymer was reported for cell imaging and PDT. 159 The P3HT was first assembled with poly(styrene-comaleic anhydride) (PSMA) to form globular nanodot assemblies and then DCM-based glycoconjugates with mannoside and galactoside were incorporated to reach multivalent glyco- dots. The glyco-dot with galactose could only recognize and visualize HepG2 cells, while the one with mannose selectively recognized MDA-MB-231 cells, as displayed in the intracellular fluorescence imaging (Figure 56). Moreover, both nanomaterials could also be used in anticancer therapy by releasing ROS under light irradiation. Dots containing galactose and mannose resulted in selective cell death of HepG2 and MDA-MB-231 in a concentration-dependent manner, and these two nanoparticles were not toxic to a healthy cell line. 

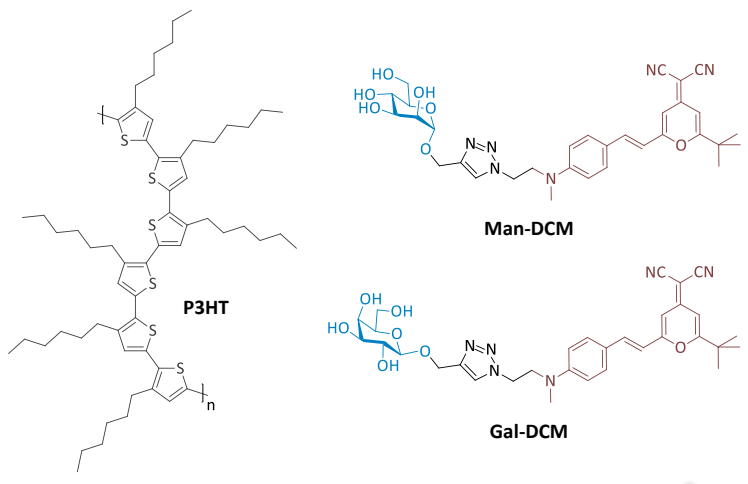
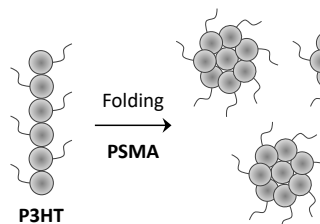

P3HT nanodots
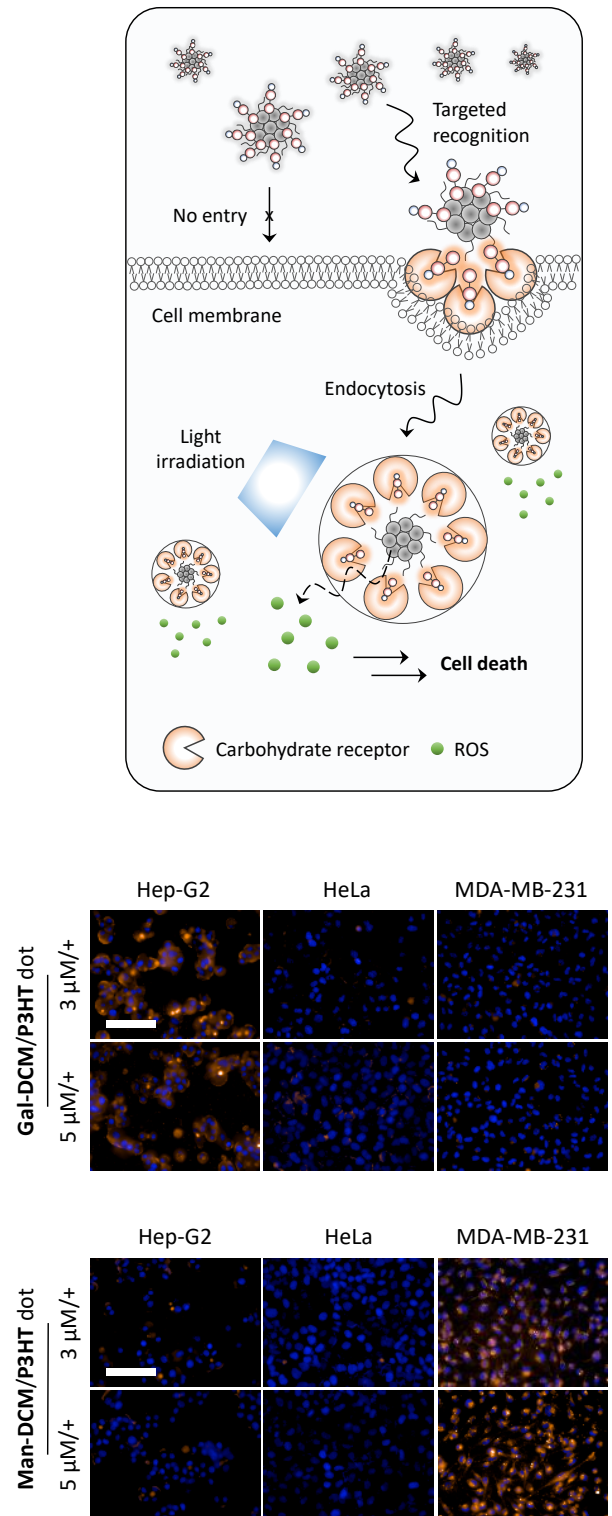

Figure 56 Structure of $\mathrm{P} 3 \mathrm{HT}$ and two glycosylated DCM-based probes, and the self-assembly with PSMA to the nanodots then the glyco-dots. Mechanism towards endocytosis and cell death. The imaging in two cells with receptors of galactose and mannose respectively and in control HeLa cells using two glycodots. Reproduced from ref. ${ }^{159}$ with permission from the Royal Society of Chemistry, copyright 2017.

A glyco-nanovesicle composed of a glycolipid (Lac-SS-DCM) in which the hydrophobic tail is the DCM moiety was designed for real-time NIR monitoring of non-invasive cellular imaging, drug release and targeted cancer therapy. ${ }^{160}$ This work covered almost all the functions of the DCM-based materials in cells discussed before. After self-assembly into vesicles, the lactose (containing galactose unit) located at the surface will trigger endocytosis indicating good hepatoma cell-targeting ability. Intracellular GSH could turn on the fluorescence because of the cleavage of the disulfide bond present in the glycolipid aglycon and thus cell imaging was realized. Moreover, when the anticancer drug DOX was loaded in Lac-SS-DCM vesicles, a perfect drug delivery system to HepG2 cells was appeared with high selectivity and reduced side effects, thus realizing the targeted cancer therapy (Figure 57).

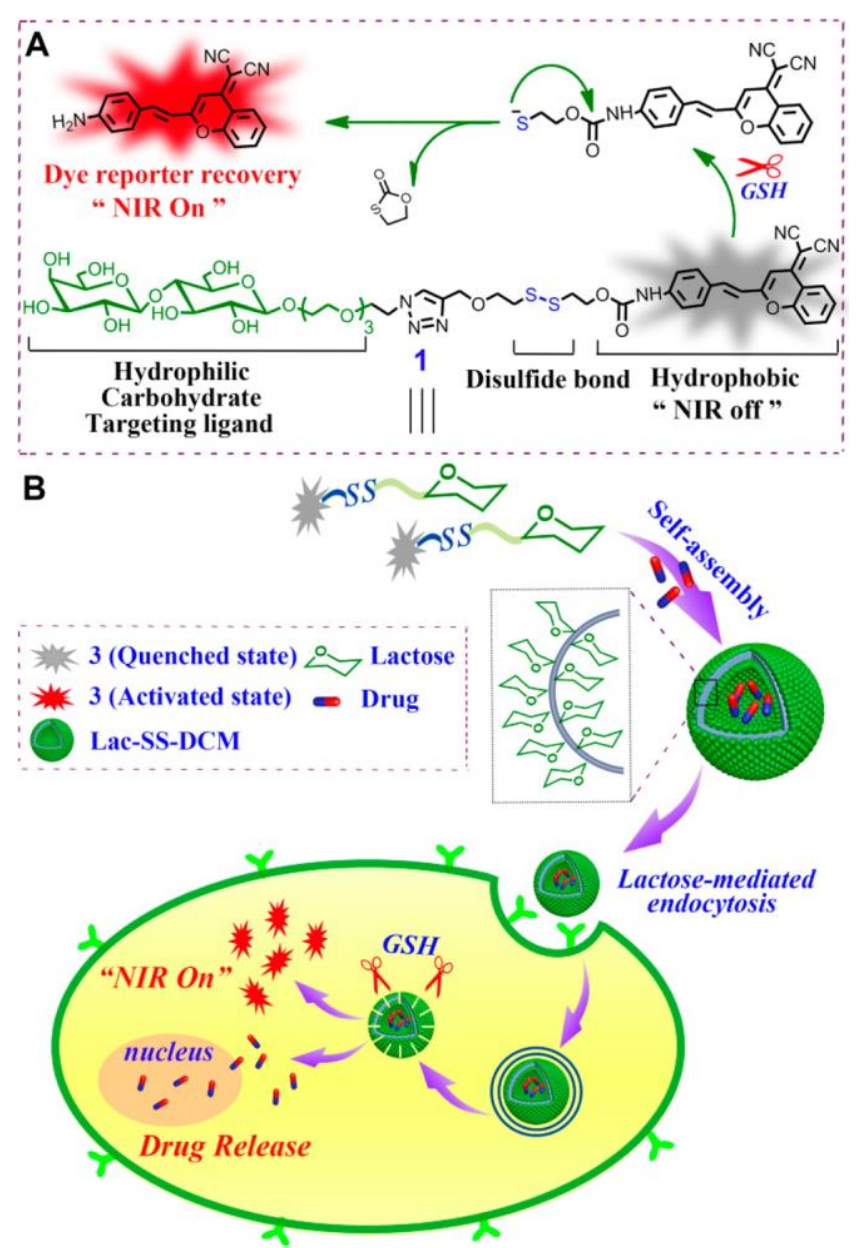

Figure $\mathbf{5 7}$ Illustration of the self-assembly, lactose-mediated endocytosis, NIR fluorescence, and drug release of a glyco-nanovesicle Lac-SS-DCM. Reproduced from ref. ${ }^{160}$ with permission from the American Chemical Society, copyright 2016.

A single DCM-based molecule (DCPO) and its glycoconjugate with glucose have been shown as probes with NIR fluores- 
cence and application in cancer cell imaging. ${ }^{161}$ The oligoethyleneglycol chain of the glycoconjugate varies in length, and the study included human cancer cells MCF-7, MKN-45, and HeLa. The medium sized oligoethyleneglycol $(n=2)$ showed the best cellular uptake in all three cells (Figure 58). Additional studies exposing three cancer cells to this compound with different concentrations and detection of the intracellular fluorescence were also conducted, proving that it could also be used as a probe for the detection of cellular glucose uptake.

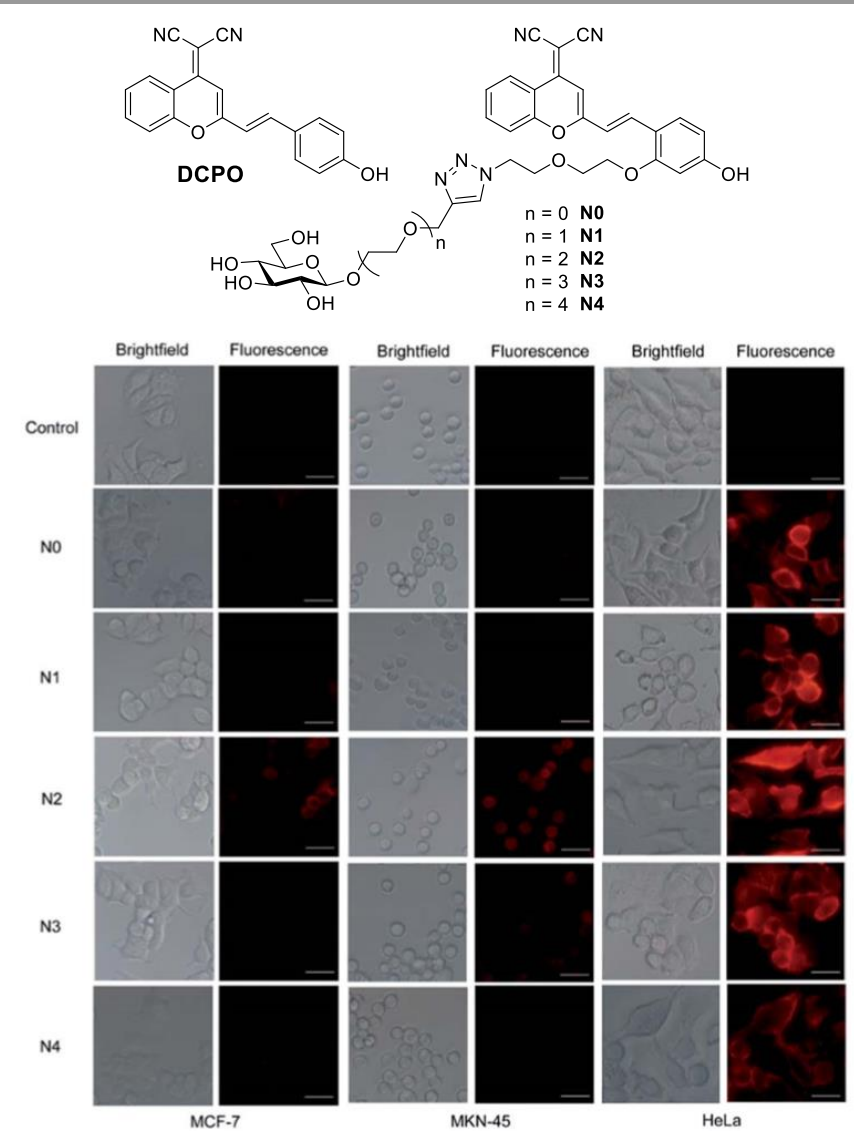

Figure 58 Structures of DCPO-based glyco-probes with different oligoethyleneglycol chain lengths. The imaging in three cancer cells using all the probes with different chain lengths. Reproduced from ref. ${ }^{161}$ with permission from the Royal Society of Chemistry, copyright 2016.

The imaging of influenza viruses using DCM-based glycoconjugates based on 2D glycosheet was reported. ${ }^{162}$ The DCMbased probes conjugated with ?-2,3-sialyl-lactose and cyanemitting coumarin (CMN) conjugated with ?-2,6-sialyl-lactose were adsorbed together on 2D $\mathrm{MoS}_{2}$. The resulting coassembly could selectively recognize influenza virus H10N8 whose hemagglutinin $\mathrm{H} 10$ is selective for ? 3 -2,3-sialyl-lactose. Another DCM-based glycoconjugate with ?-2,6-silaoside linkage interacted specifically with H1N1. Such selectivity was further confirmed by fluorescence "turn-on" detection and fluorescence imaging (Figure 59). However, when the probes were incubated with the "wrong" virus, the fluorescence signal could not be detected.
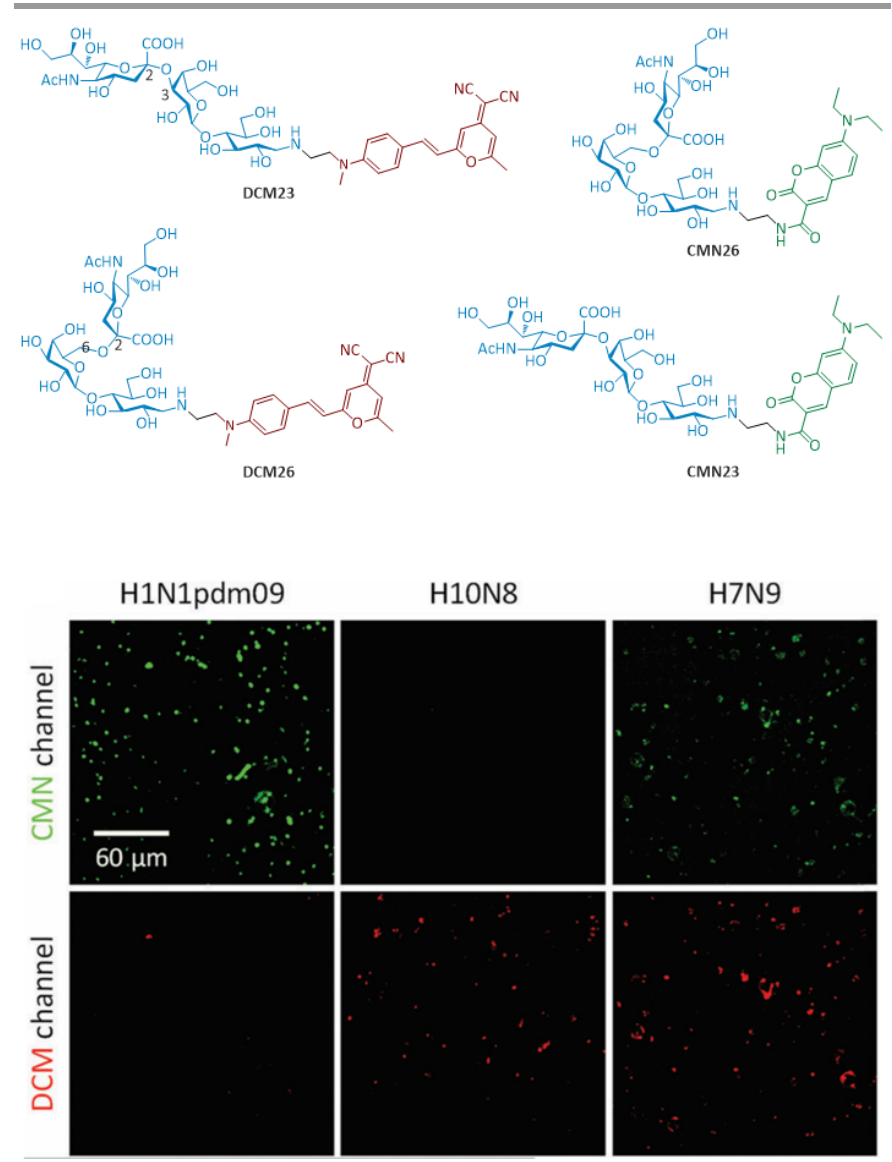

Figure 59 Structures of glycoconjugates based on DCM and CMN linked by $\alpha-2,3-$ sialyl-lactose or control carbohydrate. The imaging using 2D MoS 2 DCM and CMN glycosheets with three kinds of virus H1N1pdm09, H10N8 and H7N9. Reproduced from ref. ${ }^{162}$ with permission from the Royal Society of Chemistry, copyright 2017

Detection of $\beta$-galactosidase activity in colorectal tumour using real-time tracking and in vivo visualization with a ratiometric and light-up NIR DCM-based fluorescent probe was also reported. ${ }^{152}$ The DCM-based probe was acting as a fluorescence reporter with a $\beta$-galactoside cleavable moiety upon action of the $\beta$-galactosidase, leading to the corresponding phenolate. A clear change happened in the emission wavelength from yellow to red/NIR. The DCM-based probe could accurately monitor intracellular endogenous $\beta$-galactosidase distribution in transfection living cells, allowing real-time bio-imaging of the enzyme's activity in nude mice with colorectal tumour (Figure $60)$. Applications in cancer diagnosis for human can be expected. 

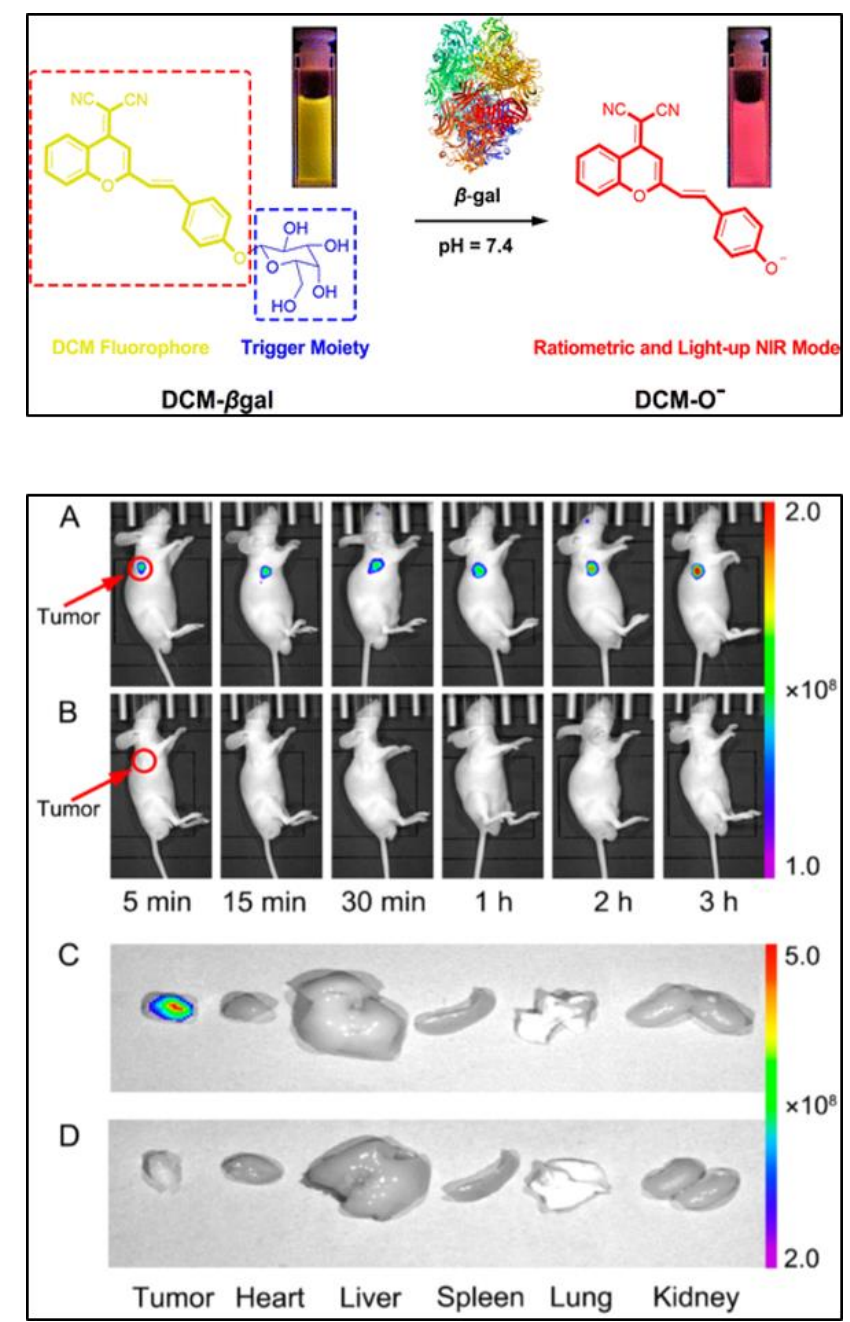

Figure 60 Illustration of the detection of $\beta$-galactosidase activity using a DCM-based galactosylated probe (top). Imaging of $\beta$-galactosidase activity in tumor-bearing nude mice after tumor injection (A,B). Fluorescence images of the main internal organs after anatomy (C,D). Reproduced from ref. ${ }^{152}$ with permission from the American Chemical Society, copyright 2016.

\subsection{Porphyrin- and phthalocyanine-based glycoconjugates}

PDT is a non-invasive technique used for the treatment of cancer. ${ }^{163-168}$ It is based on the tumour-localised generation of singlet oxygen by irradiation of photosensitisers, leading to oxidative damage of essential structural components (lipid, amino-acid and nucleic acid) and hence to cell death. Porphyrin and porphyrin-like phthalocyanines are chemically stable, resistant to degradation and relatively unchallenging to synthesize. They have an important absorption maximum between 300-400 $\mathrm{nm}$ and another absorption band fitting the biological windows in the red region of the visible spectrum. Properly metallated and substituted phthalocyanines (Pcs) are among the most appropriate photosensitizers to be used in PDT. ${ }^{169-172 ~ P c ~ s k e l e t o n s ~ a r e ~ e s s e n t i a l l y ~ h y d r o p h o b i c ~ d u e ~ t o ~}$ intermolecular interactions between the macrocycles, which decrease the water-solubility of the molecule. However, Pcbased glycoconjugates are particularly attractive due to the increased water-solubility of the photosensitisers and the potential selective recognition toward cancer cells based on carbohydrate-lectin recognition.

Nowadays, the main fluorescence technique used to study carbohydrate-protein interactions is based on the introduction of fluorescent labels nearby the binding site of lectins and subsequent monitoring of the modification of fluorescence upon saccharide binding. However, this strategy includes several issues, such as the reduction of the sensitivity or selectivity depending of the relative position of the label to the active site as well as potential allosteric changes of the protein. To overcome these drawbacks, a novel approach to study the interaction between ConA and porphyrin-based glycoconjugates was designed. ${ }^{173}$ Porphyrin-based glycoclusters (Figure 61) would aggregate in water resulting in low fluorescence intensity due to ACQ effect. Upon addition of ConA, aggregation would be disrupted leading to soluble species of glycoclusters/lectins complexes, thus generating a significant increase in fluorescence. Addition of mannose (the natural ligand of ConA) to the glycoclusters/lectins complexes releases the porphyrinbased glycoclusters with partial quenching of fluorescence due to re-aggregation.

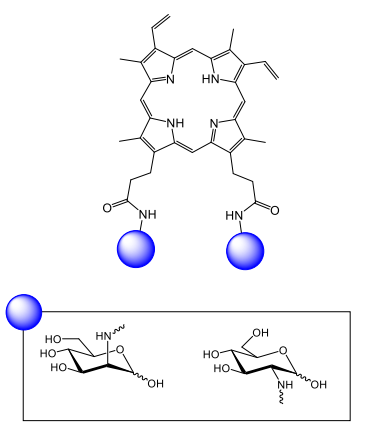

Figure 61 Structures of the porphyrin-based glycoconjugates.

The same approach was used to investigate the ability of porphyrin-based glycodendrimers to bind ConA (Figure 62). ${ }^{174}$ Interactions of aggregated non-fluorescent porphyrin glycoconjugates with ConA induce a strong fluorescence signal, which is quenched by their displacement from ConA binding sites by methyl $\alpha$-D-mannopyranoside. Interestingly, the authors have highlighted non-specific interactions of the porphyrin core with the lectin through hydrophobic interactions. However, in all studied systems (free in solution, immobilized, monolayers or liposomes), the specific carbohydrate-lectin interaction appeared to contribute more significantly than the non-specific hydrophobic porphyrin-lectin interaction as confirmed by surface pressure measurements, dynamic light scattering and QCM-D. 


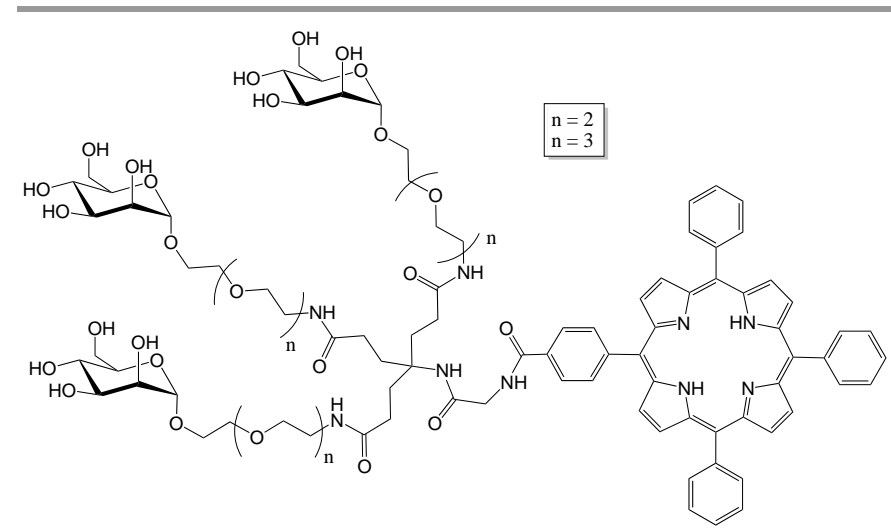

Figure 62 Porphyrin-based glycocluster used for the detection of ConA.

Other Pc-based glycoconjugates have been synthesized with three tetrasubstituted Zn(II)-Pcs displaying with galactosides or glycerol on peripheral positions or glycerol on non-peripheral positions (Figure 63). ${ }^{175}$ The electronic absorption spectrum analysis has demonstrated that all glycoconjugates are water soluble in the micromolar range, however glycerol-based Pcs have shown similar degree of aggregation, whereas glucosylated Pcs exhibited significantly less aggregation behaviour. Differences in aggregation were also observed in their photoactivity since no fluorescence images have been detected by fluorescence microscopy using either glycerol-based Pcs incubated with HT-29 cells, images showing the uptake of the Pcbased glycoconjugate were obtained with glucosylated Pcs.

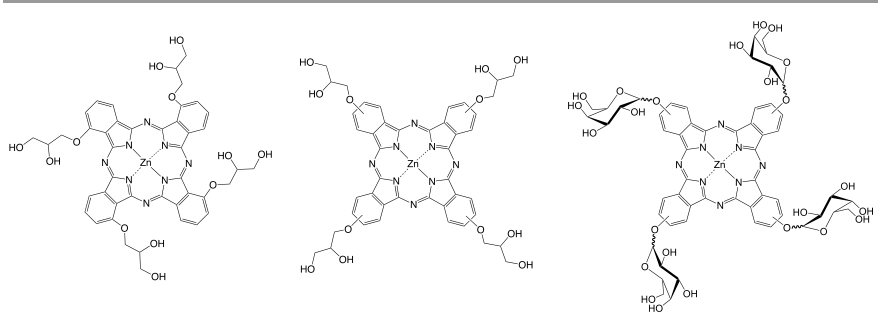

Figure 63 Structures of the PC-based glycoclusters.

Amphiphilic glycosylated $\mathrm{Ni}(\mathrm{II})$-Pcs have been designed with six hexylthio hydrophobic chains following an $\mathrm{AB}_{3}$ type substitution pattern. ${ }^{176}$ Variations of the hydrophilic part (Glc, Gal, Man or Lac) and the linker arm (with or without 1,2,3-triazole) have been considered (Figure 64). The IR spectra of the same Pc bearing different carbohydrate units have shown characteristic bands corresponding to each residue (Glc, Gal, Man or Lac). The spectra of the glycoconjugates with or without the 1,2,3-triazole moiety were found very similar. Finally, UV-Vis analyses have demonstrated that these structural variations did not influence the maximum absorption wavelengths.

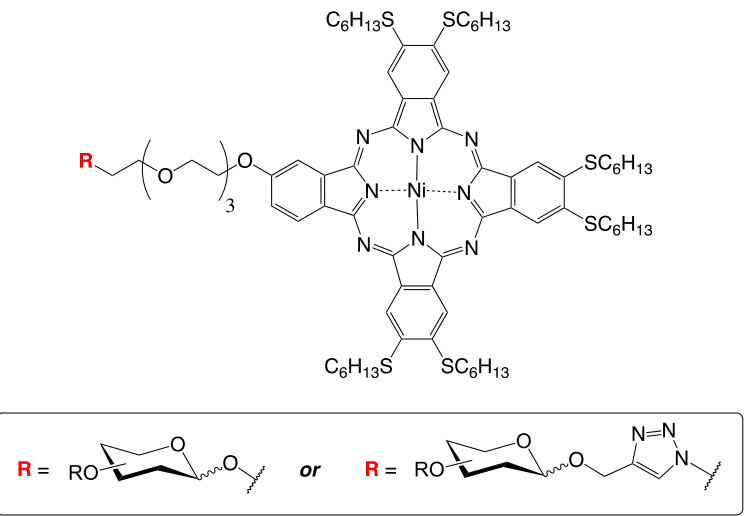

Figure 64 Structures of the amphiphilic glycosylated Pcs.

Recently, the same group has reported the preparation of a new generation of glycosylated $\mathrm{Zn}$ (II)-Pcs bearing a single carbohydrate unit and replacing the hydrophobic substituents with water-soluble glycerol moieties (Figure 65) for improved water solubility. ${ }^{177}$ UV-Vis spectra of each glycoconjugate have confirmed their solubility in water and DMSO, regardless of the nature of the carbohydrate part, although partial and limited aggregation was observed in aqueous media.

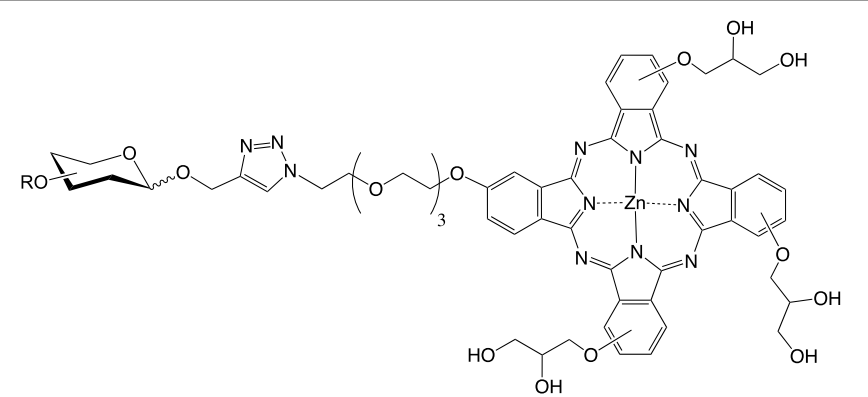

Figure 65 Structures of the glycosylated Pcs incorporating three glycerol units.

Water-soluble mono-glycosylated Zn-porphyrazines were synthesized and evaluated for their properties in PDT (Figure 66). ${ }^{178}$ The three compounds were efficient against five common cancer cell lines in vitro. The different position for conjugation of the monosaccharide did not influence the properties and the only subtle difference noticed was on the energy transfer processes and concentration dependence for each tissue. 

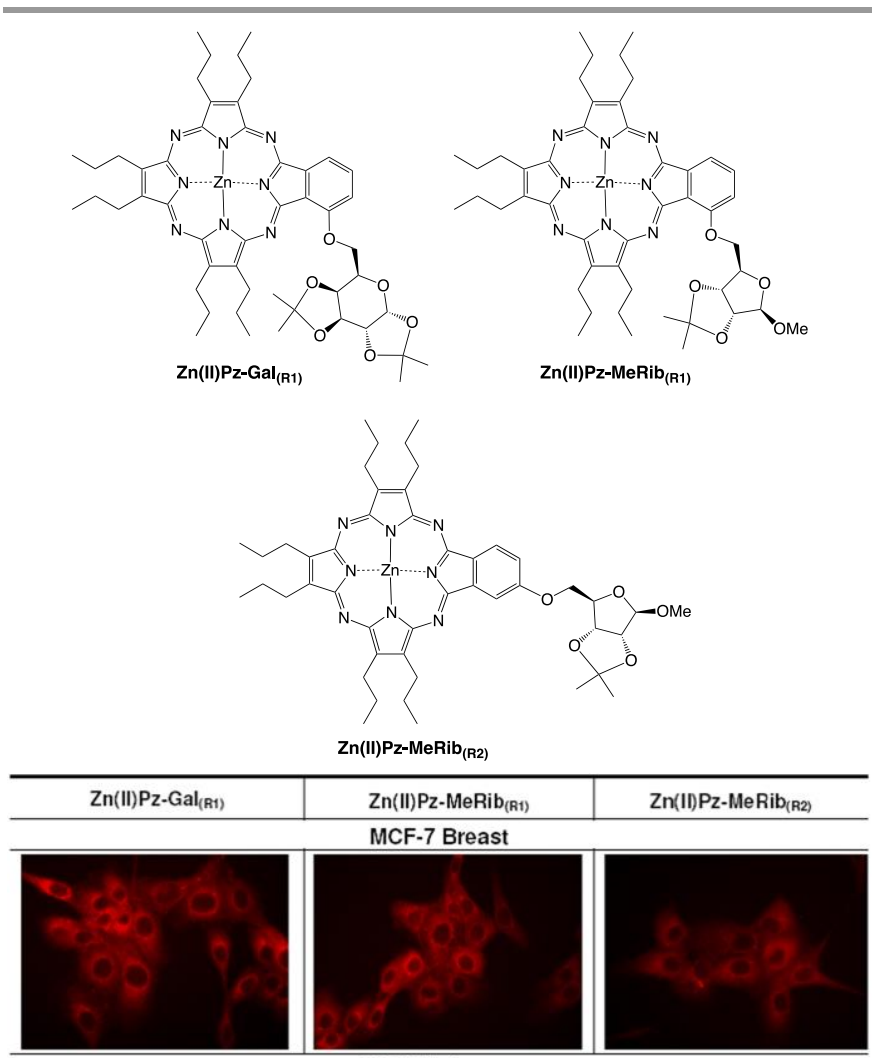

A375 Melanoma
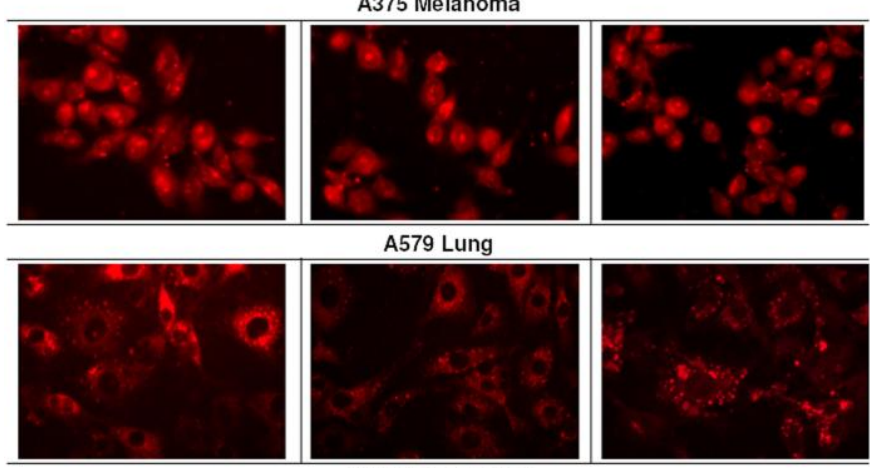

A579 Lung
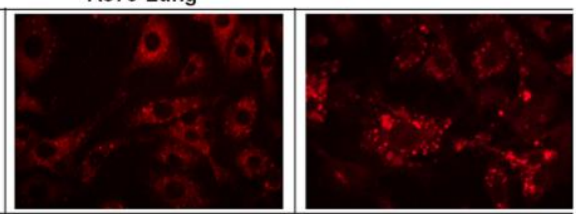

SNO Oesophageal

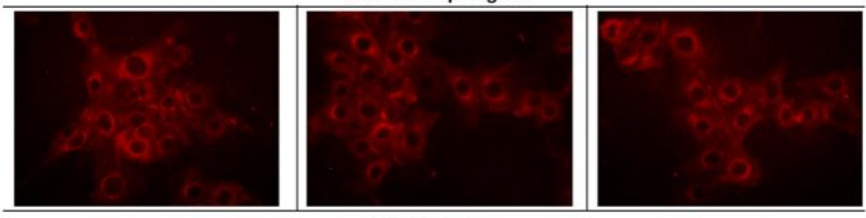

HT-29 Colon
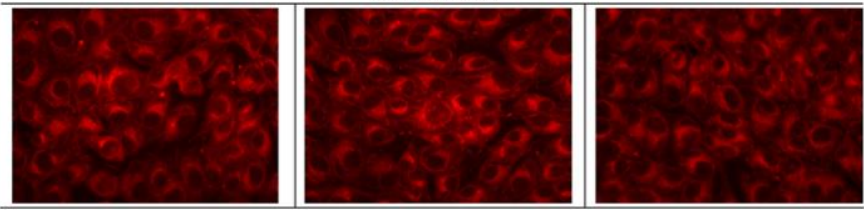

Figure 66 Glycosylated metallo-porphyrazines for application in PDT and their cellular uptake in 5 different cancer cell lines (MCF-7 breast, A375 skin melanoma, A549 lung, SNO oesophageal, HT-29 colon). Reproduced from ref. ${ }^{178}$ with permission from the Elsevier B.V., copyright 2017.

Two water-soluble glucosylated phthalocyanines were obtained by CuAAc conjugation, using a 2-methoxyethoxymethyl protecting group for the secondary positions of the carbohydrate that would resist the harsh basic cyclotrimerization con- ditions used to generate the phthalocyanine scaffold (Figure 67). ${ }^{179}$ The absorption maxima at $\lambda>680 \mathrm{~nm}$ is an encouraging property toward their application as photosensitizers in PDT. The glycoconjugate were totally soluble in DMSO with sharp Soret band ( $350 \mathrm{~nm}$ ) and Q-band ( $680 \mathrm{~nm})$. Aggregates were then observed in water characterized by the broadened and weaker Q-bands.
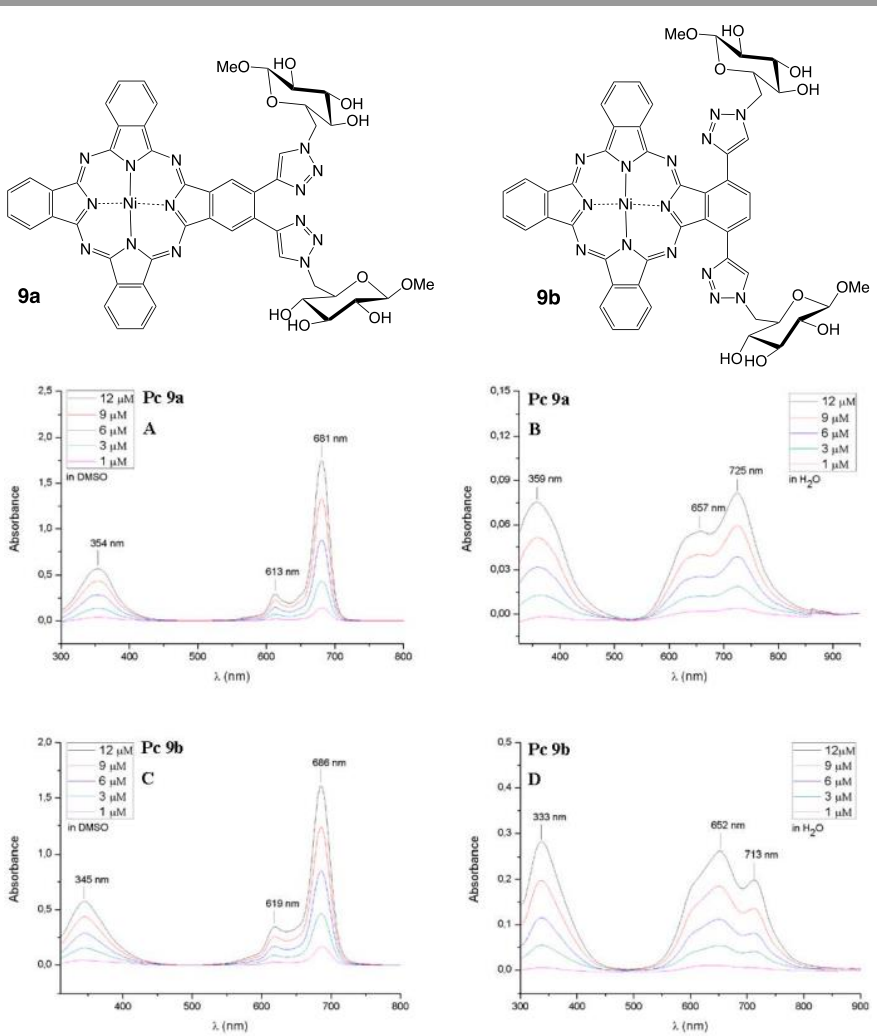

Figure 67 Glycosylated Zn-phthalocyanines and their electronic absorbance spectra measured in DMSO $(A, C)$ and water $(B, D)$. Reproduced from ref. ${ }^{179}$ with permission from the MDPI AG, copyright 2015.

\subsection{Glycoconjugates based on star-shaped fluorescent cores}

Star-shaped fluorescent glycoclusters, mainly triazatruxenebased and triphenylamine-conjugated, have been constructed for fluorescence detection of lectins. For their intrinsic photophysical and redox properties as well as their $\pi$-stacking capability, ${ }^{180}$ triazatruxene derivatives have been considerably interested especially as potential light-emitting diodes, ${ }^{181-183}$ batteries and capacitors. ${ }^{184}$ Similarly, considering their high photoluminescence and electroluminescence efficiencies, triphenylamine-based conjugates have been used for several applications including the development of optoelectronic devices ${ }^{185,} 186$ and fluorescent sensor materials. ${ }^{187,}, 188$

Fluorescence "on-off" and "off-on" probes for different lectins have been synthesized on the basis of a triazatruxene core. Indeed, it is well known that the triazatruxene moiety acts as a fluorescent probe because that it has weak $\pi-\pi$ stacking between backbones ( $\pi-\pi$ stacking interactions may induce fluorescence quenching) and has interesting fluorescence properties in water. In addition, the simultaneous association of a large hydrophobic core and flexible hydrophilic chains may have some advantageous properties. In this context, manno- 
sylated $^{189}$ and galactosylated ${ }^{190}$ triazatruxenes were efficiently synthesized by CuAAC (Figure 68).

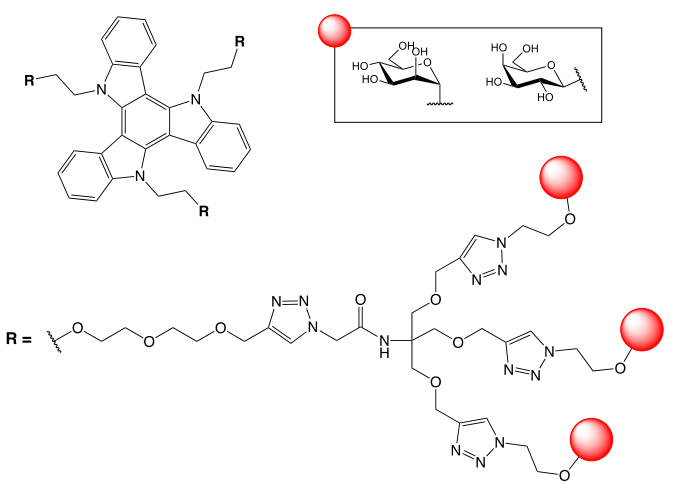

Figure 68 Structures of the mannosylated and galactosylated triazatruxenes.

These water-soluble glycoclusters were suitable for the detection of lectins using fluorescence spectroscopy. While the mannosylated glycocluster displayed a fluorescence enhancement in the presence of ConA, the fluorescence of the galactosylated glycocluster was quenched in a concentrationdependent manner with PNA. The fluorescence enhancement was rationalized by a hydrophobic interaction with lectins ${ }^{189}$ (Figure 69) whereas the quenched fluorescence was ascribed to the formation of a ground-state complex between the glycocluster and PNA, leading to static quenching. ${ }^{190}$ Although it is interesting to observe a completely reverse fluorescence response for structurally similar glycoclusters upon lectin recognition, more evidence is needed to elaborate the exact mechanism. In addition, replacement of the blue-emitting triazatruxene core by fluorophores with a longer wavelength would facilitate cellular and tissue imaging assays.

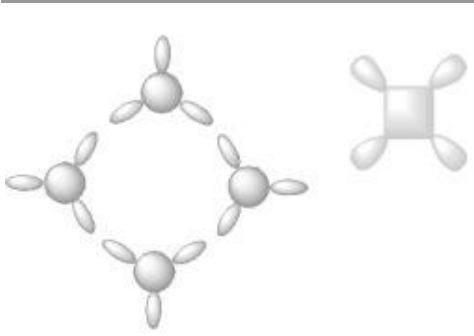

Fluorescence

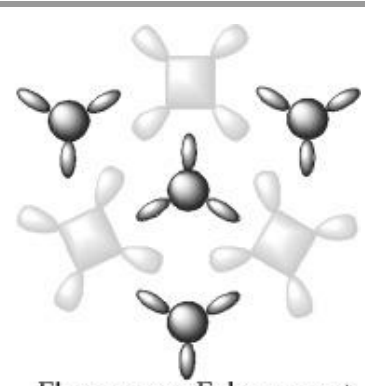

Fluorescence Enhancement
Figure 69 Illustration of the protein-carbohydrate interactions. Reproduced from ref. ${ }^{189}$ with permission from the Wiley-VCH, copyright 2013.

Triphenylamine-based glycoconjugates have been developed for receptor-targeted cell imaging (Figure 70). ${ }^{191}$ They show a two-photon absorption at $780 \mathrm{~nm}$ which has advantages in terms of deeper tissue penetration, less photo-damage and photo-bleaching. The glycopyranose moieties provided water solubility and a folic acid was also introduced to target folate receptor on cancer cells. This study demonstrates that glycosylation is an effective strategy to yield water-soluble materials with large two-photon absorption cross-section. The materials showed an enhanced fluorescence upon aggregation with
ConA, which is attributed to an energy transfer process facilitated by polymer aggregation.

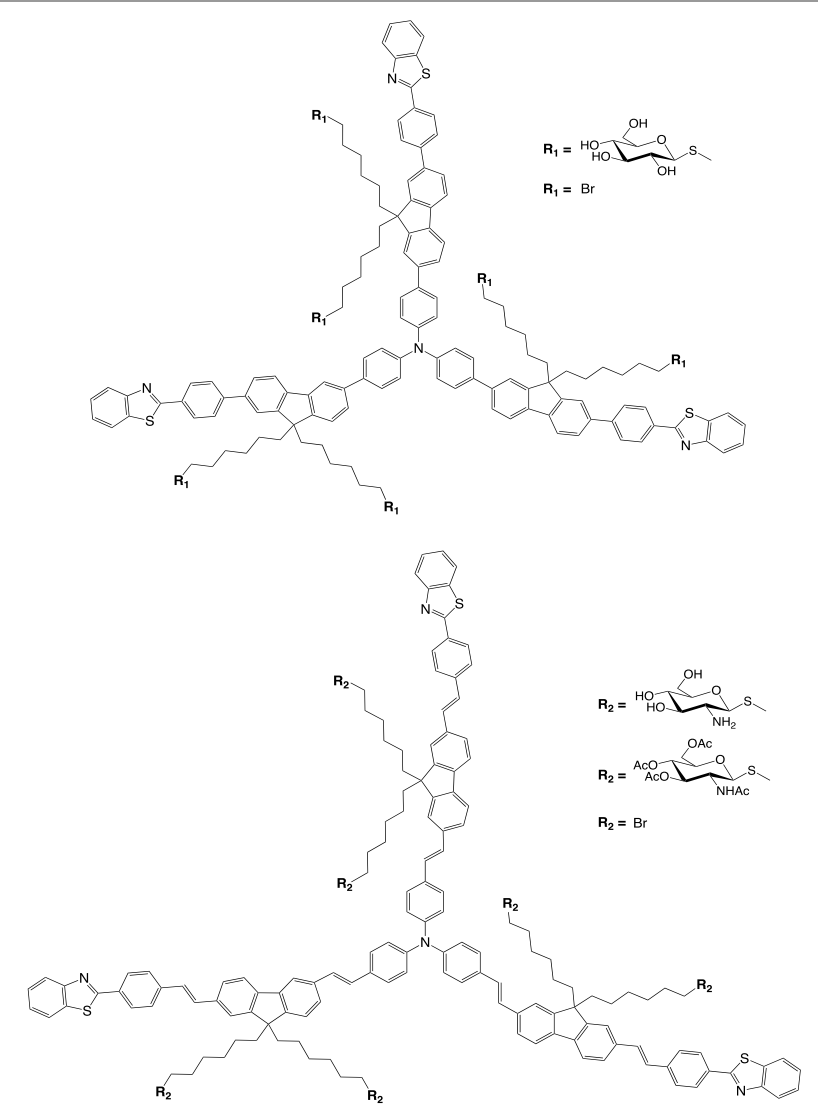

Figure $\mathbf{7 0}$ Structures of the triphenylamine-based glycoconjugates.

\subsection{Glycoconjugates based on pyrene cores}

"Click" conjugation of acetylated 2-azidoethyl lactoside with a propargyloxy-pyrene afforded after deacetylation provides a pyrene appended lactoside with two-photon fluorescence properties (Figure 71). ${ }^{192}$ While the lactose moiety could provide water solubility to the pyrene fluorescent probe, it would also allow the targeting of HepG2 cells in a two-photon imaging assay. Supramolecular assembly into nanofibers, obtained from the aggregation of the hydrophobic pyrene cores in water, led to solid state fluorescence between 400 and $600 \mathrm{~nm}$.
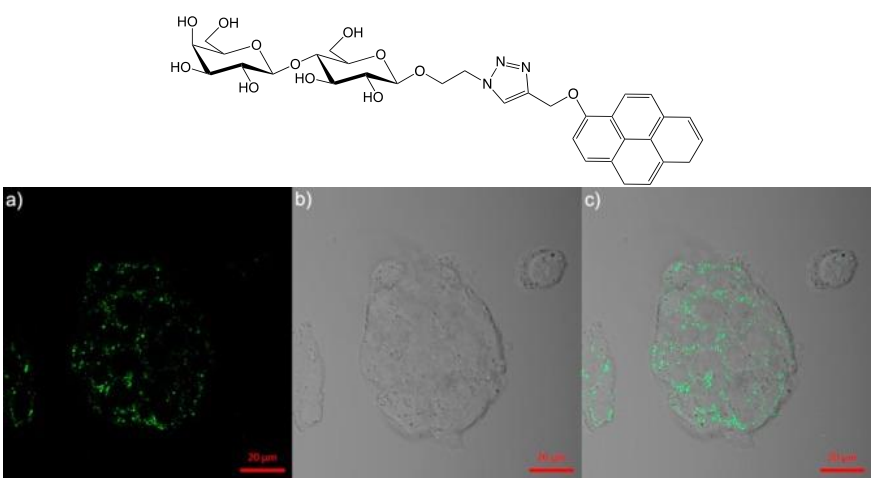
Figure 71 Structure or the pyrene-lactose conjugate and confocal microscopic images of HepG2 cells after incubation $(10 \mu \mathrm{M})$ for $2 \mathrm{~h}$ (a, excited at $780 \mathrm{~nm} ; \mathrm{b}$, bright field; c, merged images of a and b). Reproduced from ref. ${ }^{192}$ with permission from the Elsevier B.V., copyright 2018.

An amphiphilic $C$-glycoside conjugated with pyrene was investigated as a fluorescent probe (Figure 72). ${ }^{193}$ Extended ?conjugation with the pyrene moiety provides better emission properties leading to a broad spectrum. Intramolecular charge transfer (ICT) was observed and proved to be sensitive to the polarity of the medium. This probe could be used to monitor the interfacial regions of micro-heterogeneous environments.

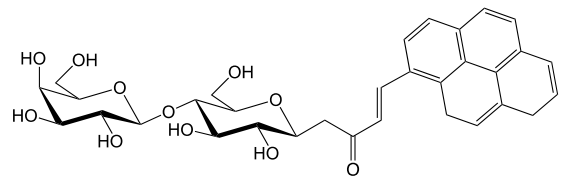

Figure 72 Structure of a $C$-lactosyl pyrene glycoconjugate as a fluorescent amphiphilic probe.

Aminopyrene trisulfonate could be used to probe the substrate scope of a plant pectin ?-galactosyltransferase (Figure 73). ${ }^{194}$ Galactose containing disaccharides were conjugated with aminopyrene trisulfonate by reductive amination and the resulting glycoconjugates were then incubated with the glycosyltransferase to monitor the acceptor substrate tolerance of the enzyme. The fluorescent label exhibited an unexpected reaction promoting effect with 0 - $(1,4)$-galactan chain extension up to 60 copies.

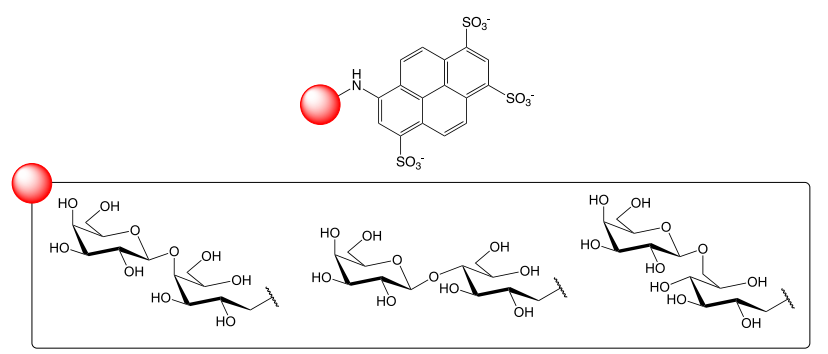

Figure 73 Structures of aminopyrene trisulfonate fluorescent glycoligands.

Water-soluble 2-C-glucosyl and O-glucosyl pyrenes were studied as photosensitizers for applications in photodynamic therapy (Figure 74). ${ }^{195}$ Water-solubility of $50-75 \mu \mathrm{g} / \mathrm{mL}$ was observed and the compounds could produce singlet oxygen in vitro. While the $O$-glucoside could be readily detected in HeLa cells and was successfully engaged in PDT, the $C$-glucoside did not enter HeLa cells.
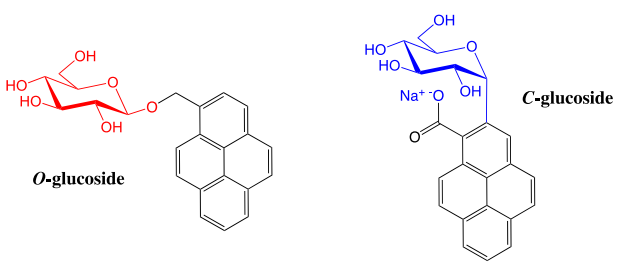

Figure 74 Structures of the pyrene $C$ - and $O$-glucosides evaluated as photosensitizers.

\section{Carbohydrate-based macromolecular glycoconjugates for biomedical applications}

\subsection{Carbohydrate as a core for one or multiple fluorescent} dyes

Due to the rigidity and stereochemical diversity of their structures as well as their low toxicity, natural abundance and biological compatibility, carbohydrates have been used as scaffolds for conjugation with fluorophores (the so-called glycoligands) toward metal ion probes and as potent chemosensors. ${ }^{120}$ 196-200 CuAAC "click" reaction has been shown as an effective strategy to construct glycoligands by forming fluorophoreappended bis-triazolyl motifs around a pyranose scaffold. The two 1,2,3-triazoles thus formed could serve as a linker, but also as a metal ion chelation site. ${ }^{200-204}$ In addition, the ion selectivity has been determined to mainly depend on the structure of the fluorophore used.

Introduction of 7-hydroxy-coumarin to the 3- and 4-positions of gluco- or galactosides (Figure 75 ) proved that the fluorescence emission of both molecules decreased after the addition of $\mathrm{Ag}^{+}$, which was mainly because of the heavy metal effect. ${ }^{205,206}$ Control studies were also conducted using other metal ions such as $\mathrm{Al}^{3+}$, $\mathrm{Pb}^{2+}, \mathrm{Na}^{+}, \mathrm{Mg}^{2+}, \mathrm{K}^{+}, \mathrm{Ca}^{2+}, \mathrm{Ba}^{2+}, \mathrm{Cu}^{2+}, \mathrm{Co}^{2+}, \mathrm{Cd}^{2+}, \mathrm{Mn}^{2+}, \mathrm{Ni}^{2+}$ or $\mathrm{Zn}^{2+}$ but showing no quenching effect to the molecules, demonstrating that the quenching effect for the detection of $\mathrm{Ag}^{+}$was highly selective. Moreover, a competition experiment was further conducted, showing that the quenching of the glucose-based compound towards $\mathrm{Ag}^{+}$ might be more efficient with the presence of $\mathrm{Pb}^{2+}, \mathrm{Mn}^{2+}$ and $\mathrm{Ni}^{2+}$.

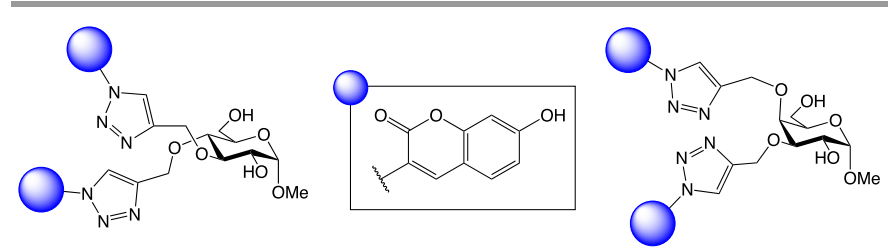

Figure $\mathbf{7 5}$ Structures of the 3,4-disubstituted 7-hydroxyl-coumarin-based fluorescent glycoligands.

The 7-hydroxy-coumarin substitution pattern around the glucopyranose ring has then been studied with the 3,4- and 2,3disubstituded derivatives (Figure 76). ${ }^{207}$ Upon selective complexation with $\mathrm{Ag}^{+}$, while the 3,4-disubstituted glycoligand displayed a quenched fluorescence due to a heavy metal effect, the fluorescence of the 2,3-disubstituted counterpart largely enhanced probably because of chelation-enhanced fluorescence (CHEF). The intracellular imaging in HepG2 cells with incubation of $\mathrm{Ag}^{+}$have also been conducted (Figure 76), further proving the sensitivity of the compound in biological conditions. This study demonstrated that the ion sensing mechanism of glycoligands can be tuned by grafting fluorophores to the different positions of carbohydrates. 

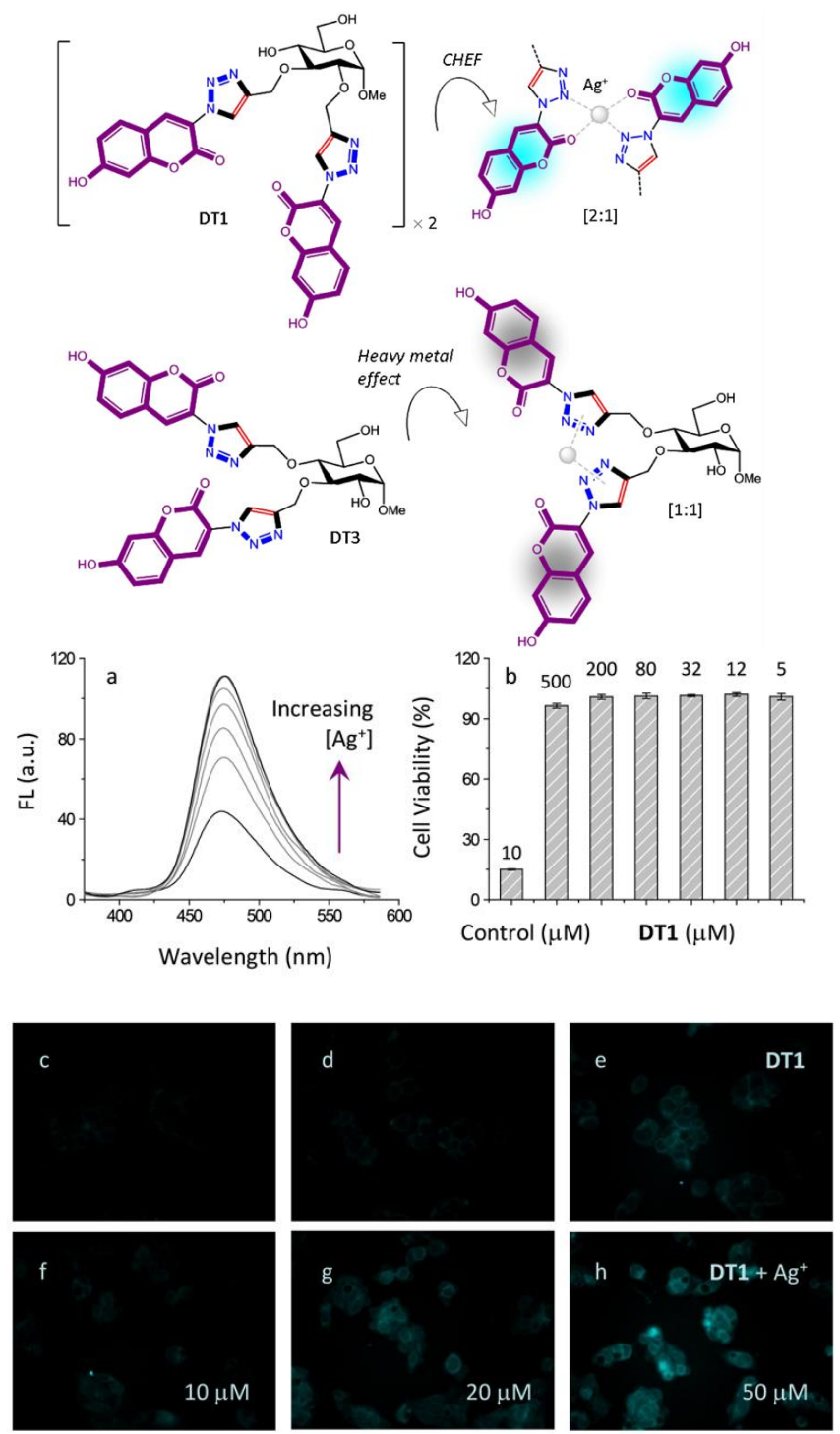

Figure 76 The explanation of $\mathrm{Ag}^{+}$binding affecting differently two probes with different substitution patterns. (a) Fluorescence spectrum of DT1 with increasing $\mathrm{Ag}^{+}$in solution, (b) cell viability and (c-h) fluorescence images of HepG2 cells in the presence of probe DT1. Reproduced from ref. 207 with permission from the Nature Publishing Group, copyright 2014.

Both glycopyranose-based 7-hydroxy-coumarin conjugates were based on a previous report ${ }^{208}$ describing the synthesis of di-7-hydroxyl-coumarin substituted glycosides with different substituting positions. The glycoligands containing three different carbohydrate moieties (glucose, galactose and mannose) displayed fluorescence emission and could be used for ion detection.

Similar 3,4-bis-conjugation with $\alpha$-ketoacid ${ }^{209}$ or amino-acid ${ }^{210}$ was reported on a $C$-aryl glycoside scaffold toward the detection of $\mathrm{Ni}^{2+}$ and $\mathrm{Cu}^{2+} .209,210$ The ketoester derivative (Figure 77) displayed a significant decrease of fluorescence upon addition of $\mathrm{Ni}^{2+}$ while it remained stable when other cations were used. Moreover, apart from the ion detection, this molecule could also display remarkable inhibitory activity on protein tyrosine phosphatase 1B (PTP1B) implicated in type 2 diabetes. ${ }^{211}$ The fluorescence of the phenylalanine residue introduced on the $C$ aryl glucoside (Figure 77) was largely quenched upon addition of $\mathrm{Cu}^{2+}$. Moreover, the further addition of cyanide would lead to the fluorescence recovery of the probe, thus providing a fluorescence "off-on" $\mathrm{Cu}^{2+}$ sensor.
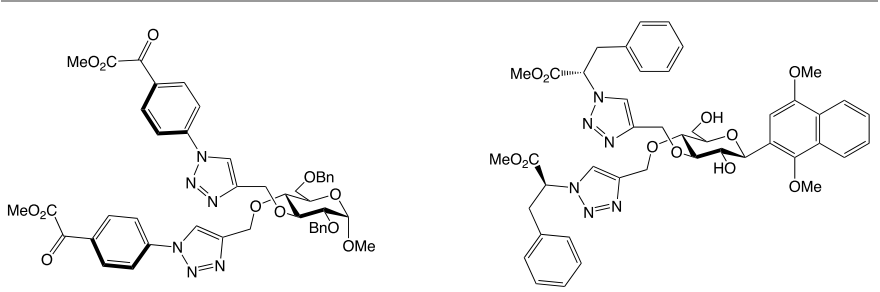

Figure 77 Structure of the $\mathrm{Ni}^{2+}$ and $\mathrm{Cu}^{2+}$ selective fluorescent probes based on a 3,4-disubstitution of glucose.

3,4-Bis-conjugation with pyrene moieties led to pyreneexcimer based bis-triazolyl glycoligands is for the selective detection of $\mathrm{Hg}^{2+}$ in an aqueous solution. ${ }^{212}$ Two glycoligands were prepared based on glucose and galactose respectively (Figure 78) to investigate the 3,4-trans or 3,4-cis substitution pattern on the pyranose ring. Both the glycoligands could quench their fluorescence emission when $\mathrm{Hg}^{2+}$ was added. The galactose-based glycoligand could recognize the $\mathrm{Hg}^{2+}$ more selectively in the presence of other metal ions than the glucose-based one.
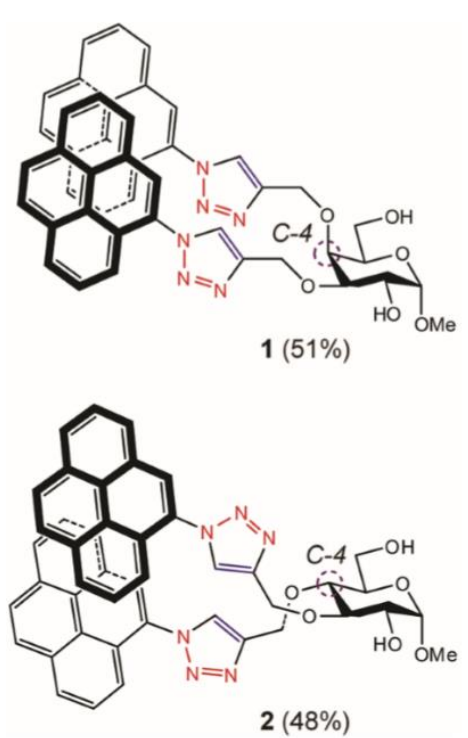

Figure 78 Structures of the glucose- and galactose-based glycoligands for the detection of $\mathrm{Hg}^{2+}$. Reproduced from ref. ${ }^{212}$ with permission from the Wiley- $\mathrm{VCH}$, copyright 2012.

4,6-Macrocyclization of an azobenzene diacid onto a glucopyranoside scaffold afforded a photoswitchable glycoconjugate with chiroptical switching properties (Figure 79). ${ }^{213}$ The macrocycle can be reversibly isomerized from $E$ to $Z$ upon UV irradiation at $365 / 435 \mathrm{~nm}$ with high photostability ( $\mathrm{t}_{1 / 2}=51$ days) for the $Z$ isomer. The macrocycle displayed gel formation capabilities in cyclohexane and ethanol with multi-stimuli responsive properties toward the expected UV irradiation at $365 \mathrm{~nm}$, but also under thermal and mechanical activation, noteworthy. 


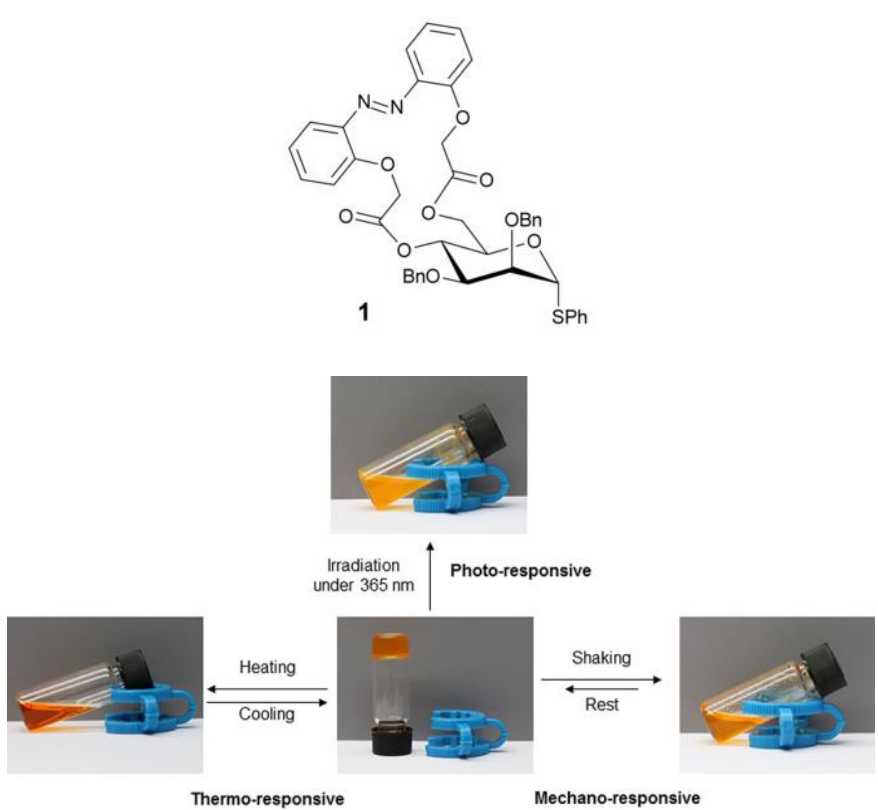

Figure 79 Glucose-based macrocyclic azobenzene as a multi-stimuli-responsive self-assembly material. Reproduced from ref. ${ }^{213}$ with permission from the Wiley$\mathrm{VCH}$, copyright 2017.

Two macrocyclic azobenzne derivatives incorporating a monosaccharide or disaccharide unit were prepared through thiourea cyclization (Figure 80). ${ }^{214}$ These water-soluble macrocycles displayed photochromic properties with $E / Z$ isomerization leading to a shape transformation of the molecule. Such isomerization could be performed up to 10 cycles without any photo-bleaching with alternating irradiations at 365 and $435 \mathrm{~nm}$. A similar macrocycle with mannoside residues and cyclized through 1,3-dialkyne obtained from a Glaser coupling was reported with comparable properties. ${ }^{215}$
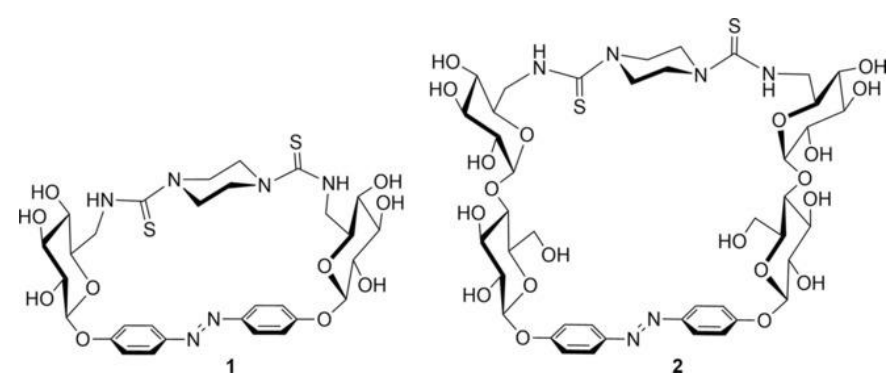

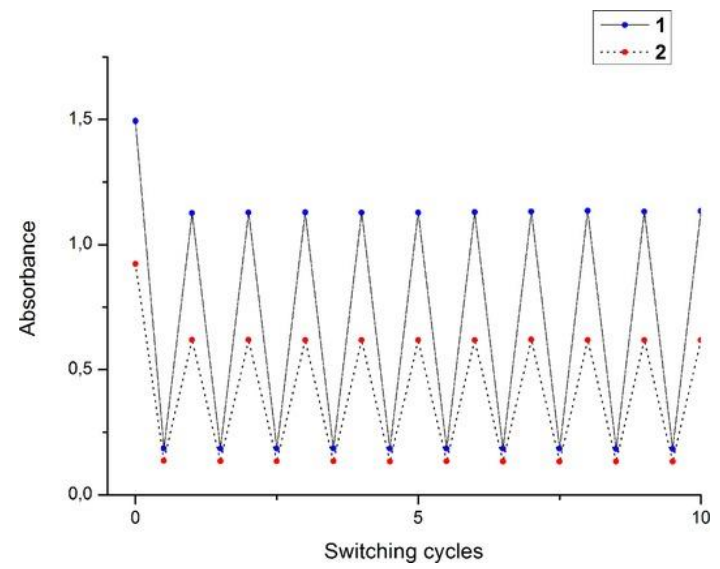

Figure 80 Glucose-based macrocyclic azobenzene derivatives for photocontrolled molecular shape. Measured absorbance after alternating photo-irradiation at $365 \mathrm{~nm}$ for $2 \mathrm{~min}$ and at $435 \mathrm{~nm}$ for $2 \mathrm{~min}$, over 10 cycles. Solid lines, blue points: absorbance at $350 \mathrm{~nm}$ for $1(0.1 \mathrm{mM}$ in water/0.5\% DMSO); dotted lines, red points: absorbance at $347 \mathrm{~nm}$ for $\mathbf{2}$ (0.05 $\mathrm{mM}$ in water). Reproduced from ref. ${ }^{214}$ with permission from the Wiley-VCH, copyright 2017.

\subsection{Cyclodextrin-based glycoconjugates}

Cyclodextrins (CDs) are water-soluble macrocycles constructed entirely by glucose units linked to each other with a ?-1,4glycosidic bond. ?-, ? 3 - and ?-CDs are composed of respectively 5,6 or 7 glucose units. ${ }^{216}$ They are found abundantly in nature, biocompatible and non-immunogenic. Moreover, a variety of selectively functionalized derivatives are commercially available or readily synthesised. Therefore, CDs have been approved as formulation excipients in food, cosmetics and pharmaceutical products. ${ }^{217}$ In addition, their cone shape creates an internal cavity largely hydrophobic that can readily host hydrophobic small molecules (e.g. adamantine or aromatic moieties), which has been used for the design of drug delivery system. ${ }^{218}$, 219 The "upper rim" composed of the primary alcohols can be chemoselectively differentiated from the "lower rim" gathering the secondary alcohols at position 2 and 3.220 On the other hand, the desymmetrization of cyclodextrins is nowadays acquired since the functionalization of six different positions on the upper rim of $\alpha$-cyclodextrin. ${ }^{221,222}$

Many studies have been devoted to the design of fluorescent sensors of highly toxic metal ions. The $\beta-C D$ glycoconjugates using CuAAC and thus generating multiple 1,2,3-triazoles are particularly attractive as chemosensors due to their ability to chelate metal ions ${ }^{223,224}$ and are widely used in many biological applications. ${ }^{216,225}$ For instance, the synthesis, fluorescence properties and complexing behaviours of $\beta-C D$ substituted by 4-(2-pyridyl)-1,2,3-triazoles have been reported as the $\mathrm{Zn}$ (II)sensitive fluorescent chelating agents (Figure 81).226 It was suggested that both triazole and pyridine moieties could participate actively in the complexation with the metal since no change was observed for acetylated or methylated CDs on the lower rim. 


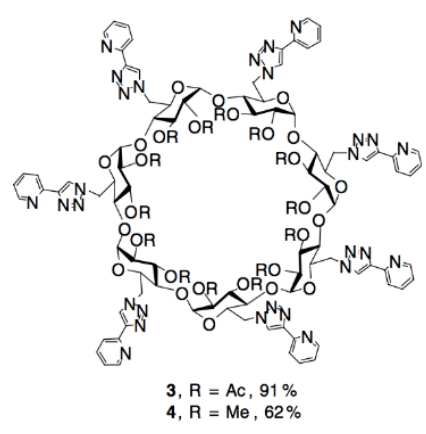

Figure 81 Structure of the $\beta-C D$-based $\mathrm{Zn}^{2+}$ fluorescent sensor. Reproduced from ref. ${ }^{226}$ with permission from the Elsevier B.V., copyright 2007.

The photophysical properties of the chromophore have been investigated in detail. 227 A photo-induced charge transfer (CT) occurring from the 1,2,3-triazole to the pyridine ring has been ascertained. The authors have studied the effects of metal ion complexation using $\mathrm{Zn}^{2+}$ and $\mathrm{Cd}^{2+}$. These cations have a soft character, and hence, should have a high affinity for the soft nitrogen atoms of the chromophore. Indeed, both nitrogen atoms of the pyridine and triazole are able to participate in the chelation of $\mathrm{Cd}^{2+}$. In addition, when the 4-(pyridin-2-yl)-1,2,3triazole chromophore was covalently linked on a calix[4]arene to form a tetrachromophoric system, a significant difference in fluorescence behaviour have been observed. In fact, upon addition of cadmium or zinc, an increase in fluorescence intensity with an inexplicable evolution of the spectrum has been obtained, while the main fluorescence band of the calix[4]arene system decreased and a new band appeared, which has been assigned to a cation-induced excimer formation. This study confirmed that the nature of the scaffold bearing chromophores could have a significant impact on the fluorescence properties.

Encouraged by this achievement, a $\beta-C D$ functionalised with a triazole-benzothiadiazole moiety was designed as a novel fluorescent sensor since thiadiazole derivatives are known to be appropriate light-emitting materials and may also be used as metal binding sites (Figure 82). ${ }^{228}$ Comparison of the fluorescence properties of the $\beta-C D$ conjugates and the single glucose-based unit have shown that both the absorbance and the emission maxima shifted to the red wavelength when the benzothiadiazole was conjugated to the $\beta-C D$. The $\beta-C D$ conjugates also displayed fluorescence quenching upon addition of $\mathrm{Co}^{2+}, \mathrm{Cu}^{2+}, \mathrm{Hg}^{2+}$ or $\mathrm{Ni}^{2+}$ while the majority of other cations did not affect the fluorescence emission. The fluorescence quenching observed using $\mathrm{Ni}^{2+}$ was attributed to a photoinduced electron transfer (PET) or a CT mechanism. Here again the formation of a triazole-benzothiadiazole-cyclodextrin/ $\mathrm{Ni}^{2+}$ complex in a 1:1 ratio has been confirmed in a fluorescence titration experiment.

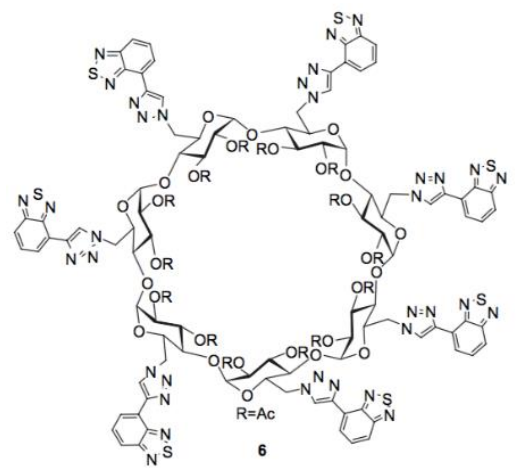

Figure 82 Structure of the metal sensing CD-based glycoconjugate. Reproduced from ref. ${ }^{228}$ with permission from the Elsevier B.V., copyright 2008.

Rhodamine-based $\quad \beta-C D$ fluorophore/adamantane-modified inclusion complex magnetic nanoparticles (TFIC MNPs) were used as probes for the detection of $\mathrm{Hg}^{2+}$ (Figure 83). ${ }^{229}$ The magnetic core of the nanoparticle was silica microspheres $\mathrm{Fe}_{3} \mathrm{O}_{4} @ \mathrm{SiO}_{2}-\mathrm{Ad} \mathrm{MNPs}$, and then the core was complexed with fluorophore-CD conjugates for detection through host-guest interactions. The nanoparticle-based sensor showed an effective and significant fluorescence increase ("turn-on") in the presence of $\mathrm{Hg}^{2+}$, and the colour of the nanoparticle would also turn to pink from brown. Then, the special interactions between $\mathrm{Hg}^{2+}$ and the nanoparticle were proved to be highly selective, due to a larger surface area and high permeability. More importantly, the magnetic nanoparticles could be easily separated and collected using a magnet. 


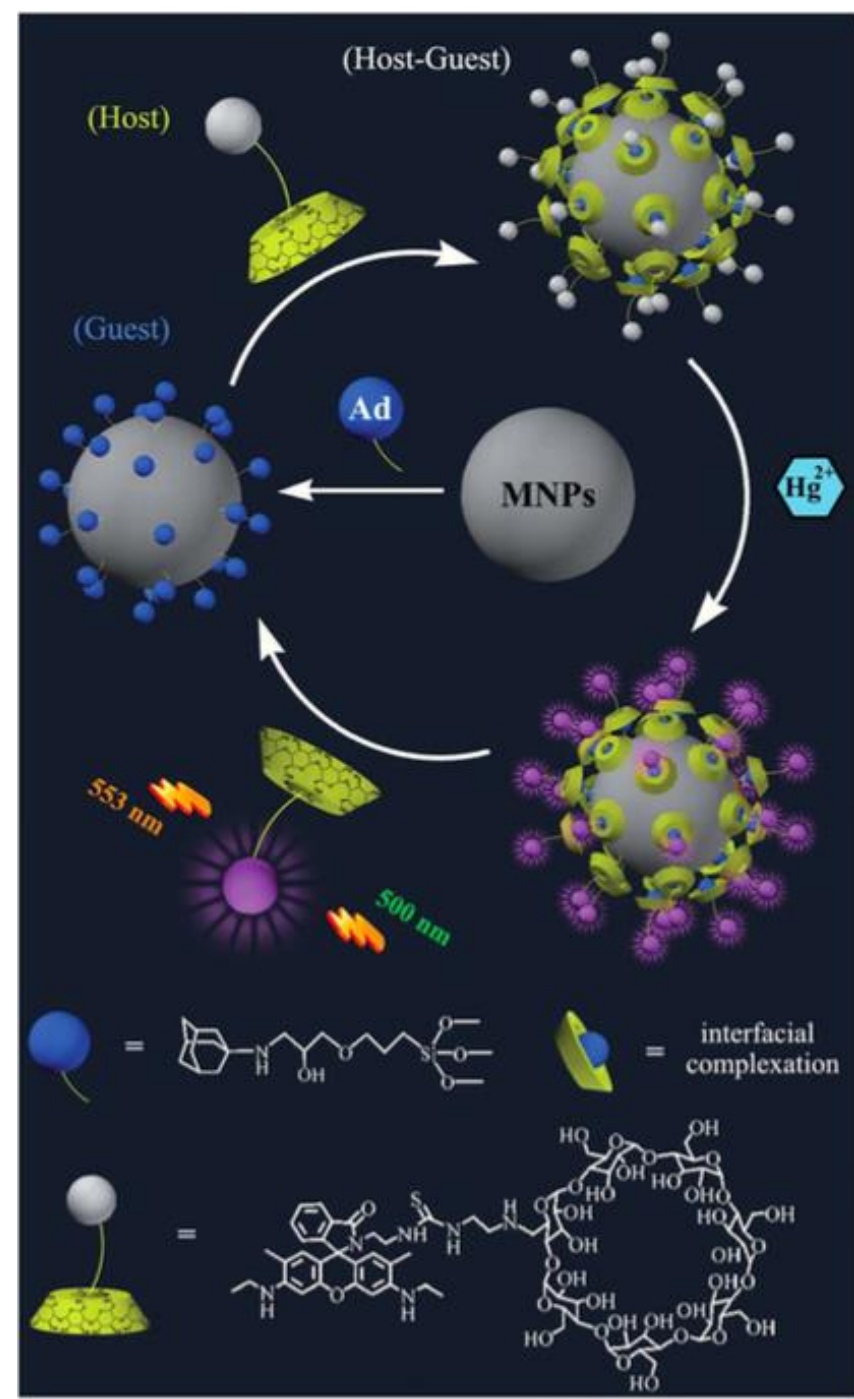

Figure 83 Chemical and schematic illustration of the preparation of TFIC MNP fluorescent sensors for $\mathrm{Hg}^{2+}$ ions. Reproduced from ref. ${ }^{229}$ with permission from the Royal Society of Chemistry, copyright 2013.

It is worth to mention that the important uses of CD-based glycoconjugates are not just limited to the detection of metal ions, they were also used in the detection of biomolecules in biomedical areas. A supramolecular complex was formed between a tubular-shaped pyrenyl- $\beta-C D$ and glycosylated rhodaminewas designed for the fluorogenic detection of lectins (Figure 84). ${ }^{230}$ The addition of the glycosylated rhodaminebased compounds led to the fluorescence quenching of rhodamine due to the formation of a 1:1 rhodamine-CD complex. Adamantane was then used as an inhibitor of the rhodamine$C D$ inclusion to investigate the complexation mechanism. Surprisingly, addition of a large excess of adamantane did not avoid the quenching of fluorescence of the rhodamine, which suggested that it was not included in the cavity of CD. The rhodamine might rather stack with the CD-based pyrenecluster via $\pi$-interactions leading to the fluorescence quenching. However, the addition of specific lectins (SBA for GalNAC and wheat germ agglutinin (WGA) for GICNAc) to the supramolecular complexes previously formed led to the fluorescence recovery of the system since lectins would disrupt the rhodamine-pyrene interactions.

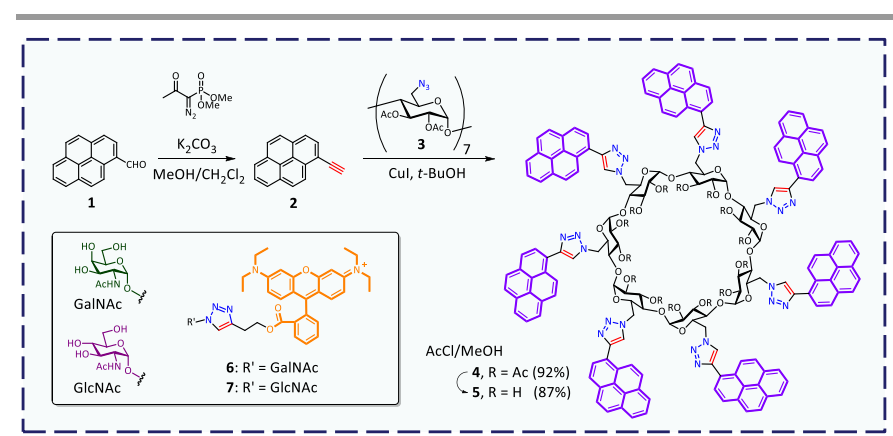

Figure 84 Structure of the pyrenyl-CD-based conjugates for the detection of lectins, and the structures of two rhodamine-based glycoconjugates used in this context. Reproduced from ref. ${ }^{230}$ with permission from the Royal Society of Chemistry, copyright 2014.

Two mannosylated CD-based glycoconjugates bearing NO photo-donors (NOPD) have been designed taking advantage of the mannose moieties for selective recognition by lectins. ${ }^{231}$ Recognition of antibiotic resistant bacteria expressing such lectins and drug delivery applications were also discussed. The binding interactions with Con $A$ between the mannosylated CDs with or without NOPD (Figure 85) as well as their efficiency were studied. The molecules without NOPD could bind efficiently to ConA. When the NOPD was introduced, a much more complex binding mode was induced and sharply modified the thermodynamic profiles of association to ConA.

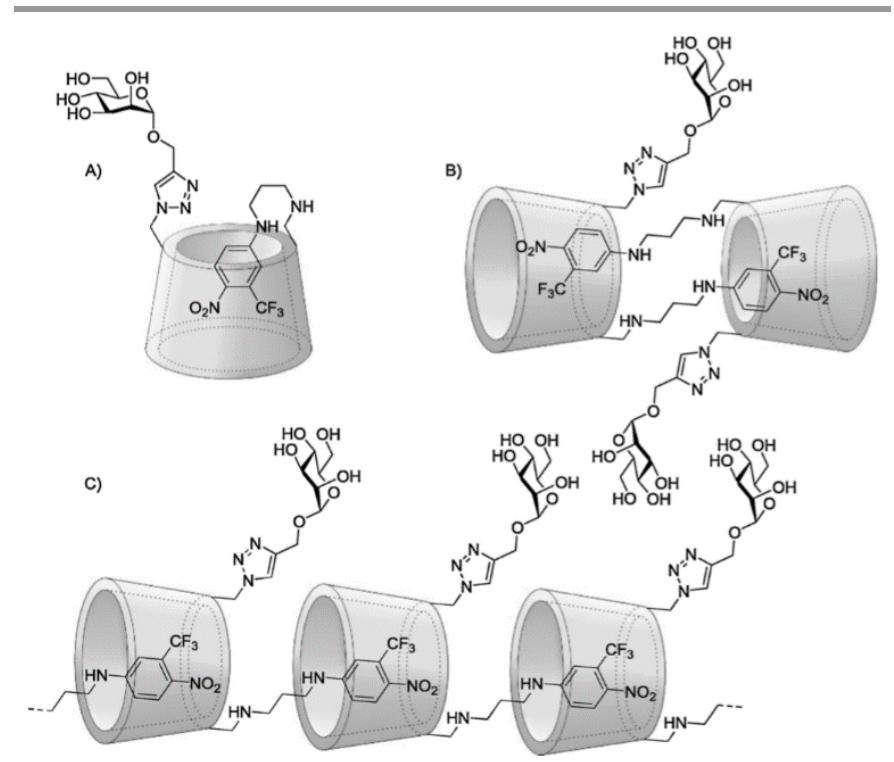

Figure 85 Structures of mannosylated CD-based glycoconjugates bearing NO photo-donors (NOPD). (a) Monomeric intramolecular self-inclusion. (b) Head-tohead dimeric complex. (c) Head-to-tail polymeric self-aggregation. Reproduced from ref. ${ }^{231}$ with permission from the Elsevier B.V., copyright 2018.

Sensing of amino acids was reported for the discrimination of enantiomers of valine and proline, based on a ligand exchange mechanism. ${ }^{232}$ A fluorescence-quenching method mediated by $\mathrm{Cu}^{2+} /$ amino acid complexes was designed for the detection of samples with high enantiomeric excess. The CD-based detecting probe was bearing a three-point metal binding site in the linker arm between the CD and a dansyl fluorophore (Figure 
86). Enantioselective fluorescence responses were observed in the presence of $\mathrm{Cu}^{2+}$ and amino acids. The sensors could improve the rapidity of the detection method as well as simplicity for the enantiomer screening.

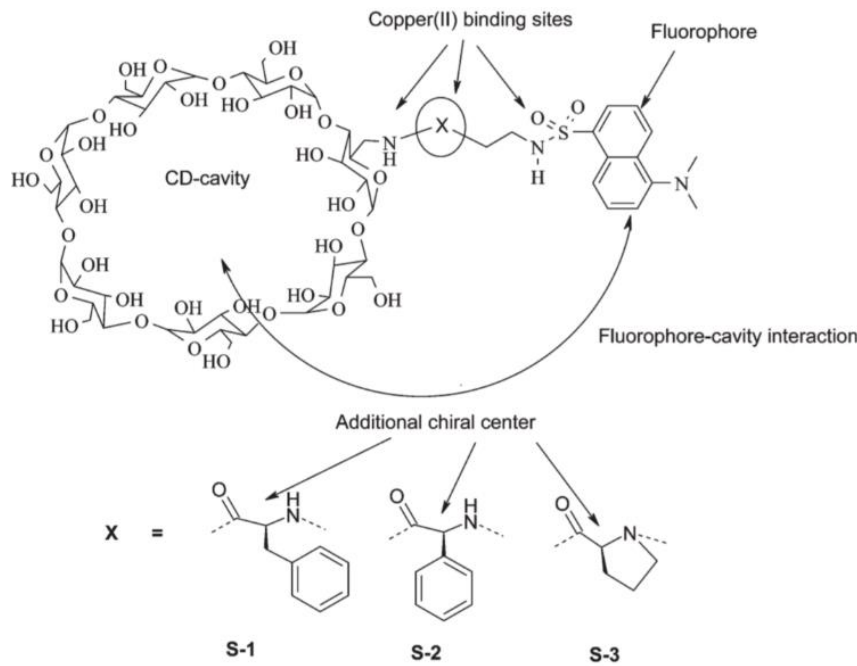

Figure 86 General scheme of the amino acid sensing enantioselective fluorescent CD-based fluorescent probes. Reproduced from ref. ${ }^{232}$ with permission from the Royal Society of Chemistry, copyright 2005.

Three CD-based systems containing different amount of fluorophore were used to compare their sensing properties and the influence of covalent versus non-covalent conjugation as well as fluorescence loading. ${ }^{233}$ One CD-based material would use the complexation of the fluorophore in the CD cavity, while the other two were containing one or two fluorophores at the "upper rim" (Figure 87) Small organic molecules which are largely aromatic were then detected through their interaction in the hydrophobic cavity of the $\mathrm{CD}$. The conjugated fluorophore units were working as transducers, responsible for fluorescence-based responses to variations in the surrounding environment upon addition of the target analyte (Figure 88).

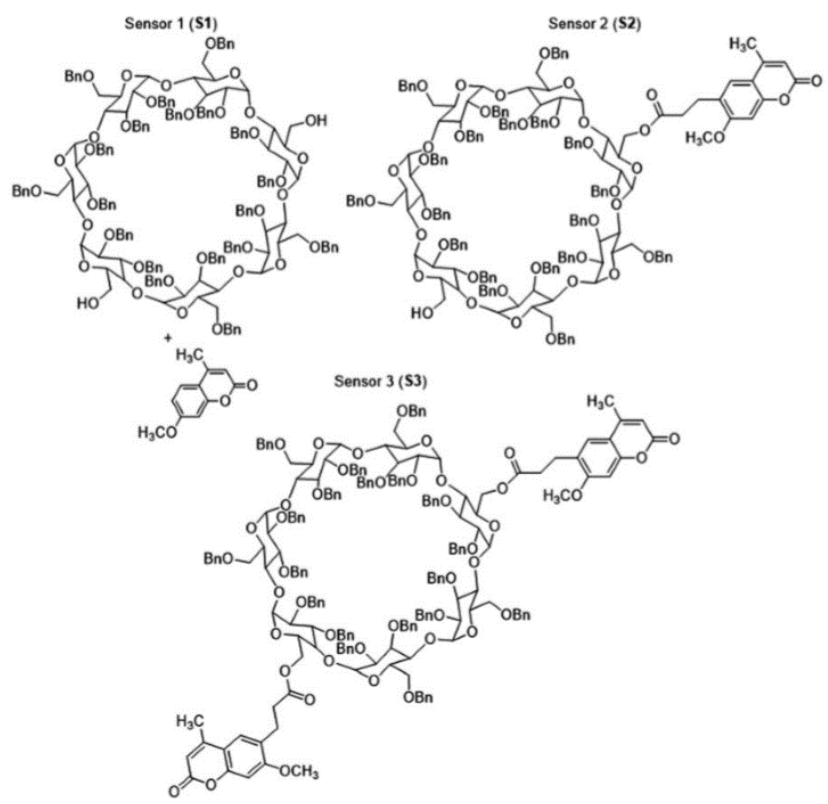

Figure 87 Structures of three CD-based sensors. Reproduced from ref. ${ }^{233}$ with permission from the Royal Society of Chemistry, copyright 2017.

Benzyl alcohol further increased its binding constant with the fluorophore-functionalized CDs compared with the native CDs. The other members of aromatic alcohols analytes displayed similar results were modifying the emission properties of the host. The classification of three isomeric (aromatic alcohols, aliphatic alcohols, aliphatic hexanes) and two analogous (DDT pesticides, PCB congeners) analyte classes was proved to be successful.

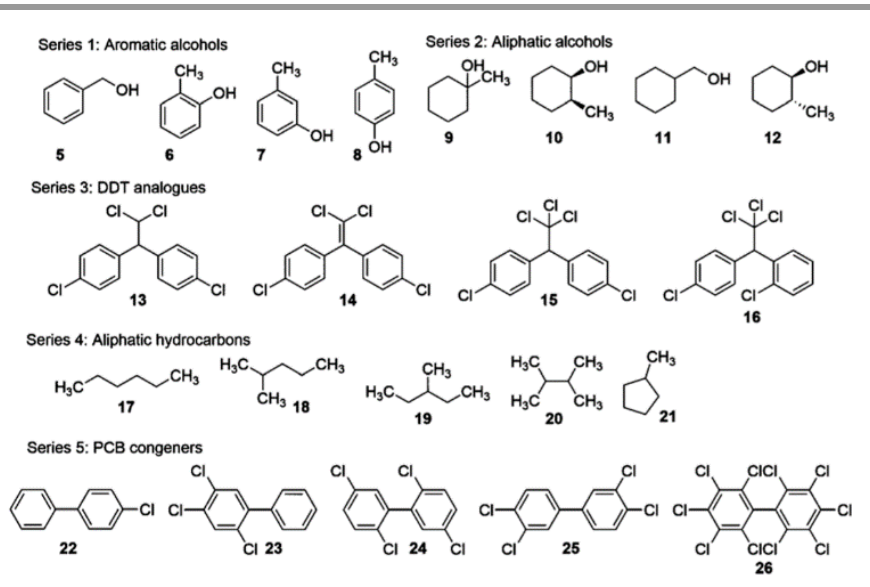

Figure 88 Structures of the analytes tested. Reproduced from ref. ${ }^{233}$ with permission from the Royal Society of Chemistry, copyright 2017.

Applications of CD-based glycoconjugates to drug delivery systems was investigated through the hydroxypropyl- $\beta-C D$ for targeted, $\mathrm{pH}$-independent controlled delivery of a drug. ${ }^{234}$ The drug whose salt shows $\mathrm{pH}$-dependent solubility would have releasing problems at high $\mathrm{pH}$ value, so the $\mathrm{pH}$-independent CD-based drug release improved dramatically the oral bioavailability of the drug. When designing drug delivery, an effective method to monitor the drug release based on fluorescence is also essentially required. This monitoring was accomplished through a FRET-based nanoparticle for the construction of the traceable drug delivery system and the real-time monitoring of the drug releasing behaviour in cancer stem cells (Figure 89). ${ }^{235}$ Cysteine conjugated with coumarin was tethered onto mesoporous silica nanoparticles (MSNs) as the carrier of drug DOX. Fluorescein isothiocyanate- $\beta-C D$ (FITC- $\beta-C D$ ) was introduced for drug loading and a FRET donor-acceptor pair of coumarin and FITC integrated within the pore-unlocking event, which enables the detection of the release of drugs from the pores. The entire molecule containing two fluorescence moieties could be reduced by GSH which is highly concentrated in cancer cells. The original molecule showed a FRET effect, inducing the fluorescence "turn-on" of fluorescein (Figure $89 \mathrm{~A}$ ). In the presence of high concentrations of GSH, the FITC- $\beta-C D$ was released and the FRET effect was no longer existing, thus turning on the coumarin fluorescence (Figure 89A). This FRET "on-off" system and the emission of coumarin was used as the real-time drug release monitoring (Figure 89B). 


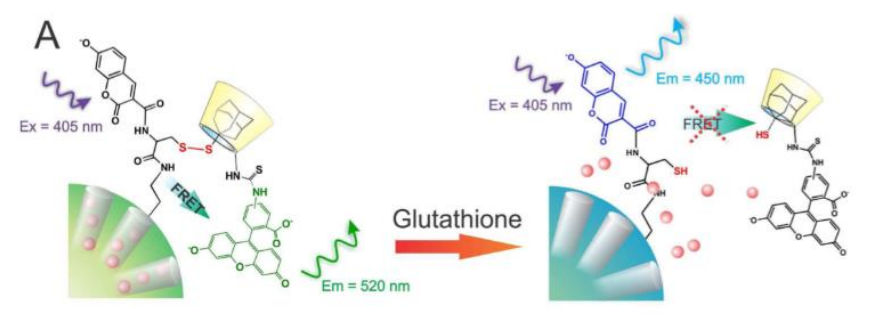

B

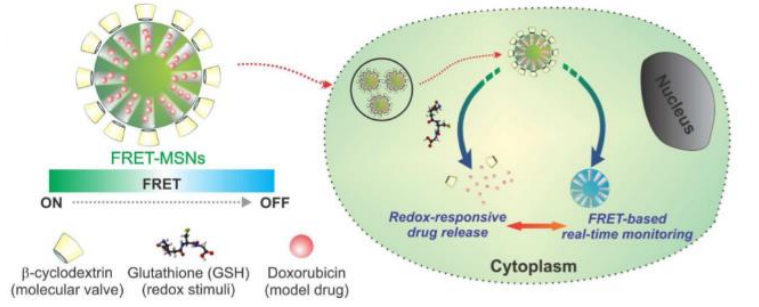

Figure 89 (A) Structure and drug release monitoring principle. (B) Celle penetration, drug release and FRET-based monitoring of drug release. Reproduced from ref. ${ }^{235}$ with permission from the American Chemical Society, copyright 2013.

\section{Polymer-based glycoconjugates for biomedical applications}

Polymeric cores are providing a plethora of scaffolds for the design of glycoconjugates with significant therapeutic potentials. ${ }^{236-238}$ These glycopolymers having both high biocompatibility and valency allow to the multivalent effect to occur, and hence, an optimal interaction with lectins or biomolecules targeted. Consequently, glycopolymers have found emerging applications in the fields of macromolecular drugs ${ }^{239-244}$ and drug-delivery systems. ${ }^{245-247}$ The recent advances in controlled polymerization reactions including reversible additionfragmentation chain-transfer polymerization (RAFT), ${ }^{248-254}$ or atom-transfer radical polymerization (ATRP) ${ }^{255}$ provide powerful tools to synthesize well-defined glycopolymers since it has been demonstrated that ligand type and ligand density need to be considered to achieve the desired biological function. Besides the high valency of polymers allowing the display of multiple carbohydrate epitopes, another advantage of this type of glycoconjugate is the intrinsic fluorescence properties of their polymeric backbone. This fluorescent glycoconjugated polymers including glyco-PPEs, glycopolythiophenes, and glycopoly( $p$-phenylene)s have been explored for biosensing applications.

Water-soluble glycosylated polymers with pendant glycodendrons were reported and their interaction with heparin was studied.256 These polymers were conjugated with glucosamine residues as cationic moieties. Heparin is a linear anionic polysaccharide and its interaction with such cationic polymers was therefore facilitated. Two glycosylated polymers bearing three (P-3) or one glucosamine (P-1) unit for each monomeric unit were synthesized and studied (Figure 90).

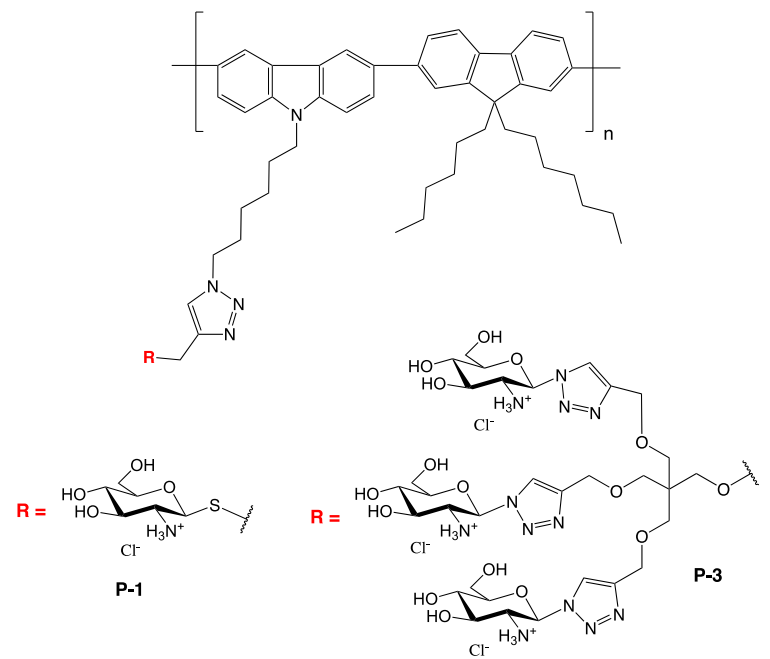

Figure 90 Structures of the triple branching (P-3) or single branching (P-1) glucosamine containing glycosylated polymers.

The combination between heparin and P-3 induced a fluorescence quenching of the intrinsic polymer backbone's fluorescence (Figure 91). Competition with protamine which bound heparin released the P-3 glycosylated polymer thus restoring the fluorescence emission. The emission of $\mathbf{P}-\mathbf{3}$ gradually decreased with the addition of heparin. At high concentrations of heparin and minimal emission of the P-3 polymer, protamine was added. The fluorescence emission of the P-1 glycosylated polymer did not decrease upon addition of heparin due to the limited ammonium binding sites for heparin. The P-3 polymer can therefore be considered as a suitable sensor for the detection of both heparin and protamine.

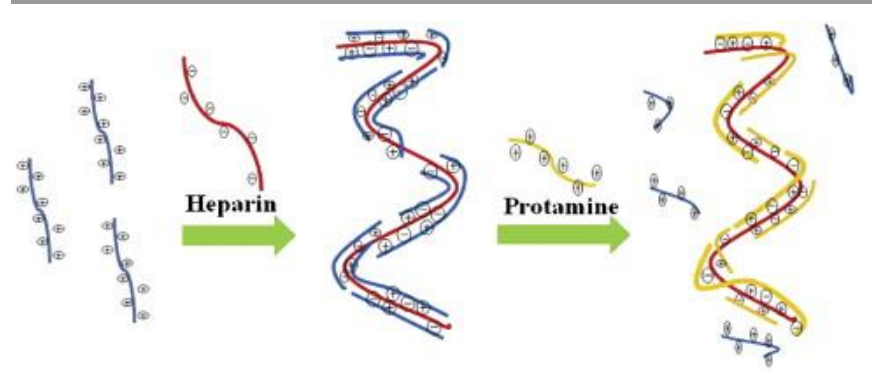

P-3

Fluorescence recovery

Figure 91 Mechanism of detection of heparin and protamine using the P-3 glycosylated polymer. Reproduced from ref. ${ }^{256}$ with permission from the Elsevier B.V., copyright 2011.

The fluorescence self-quenching of a mannosylated PPE (PPEMan) was reported upon addition of the polymer to a solution of Con $\mathrm{A}$ as a detection system for lectins. ${ }^{257} \mathrm{~A}$ mechanism for the interaction was proposed (Figure 92). With the addition of PPE-Man to ConA, more and more glycosylated polymer was combined to ConA and aggregated together, resulting in a FRET process, and inducing the further efficient fluorescence quenching. 


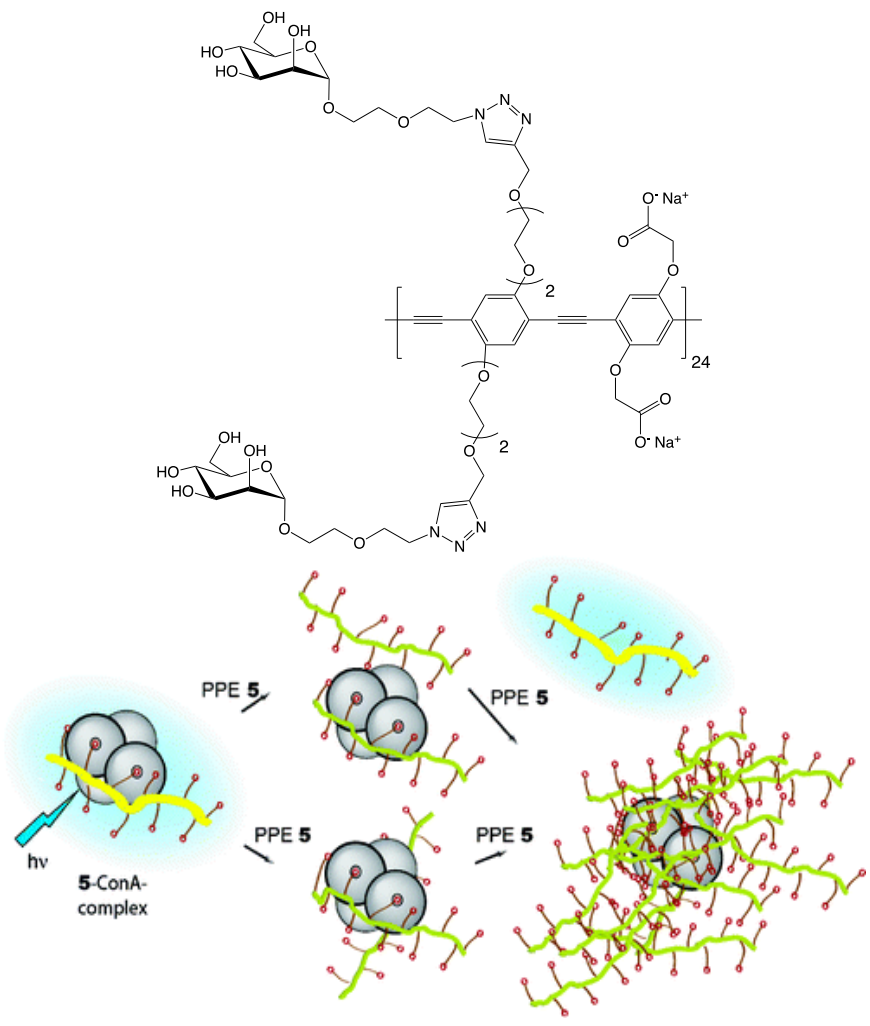

Figure 92 Structure of a PPE-Man glycosylated polymer and interactions between PPE-Man and Con A (bottom). Reproduced from ref. ${ }^{257}$ with permission from the American Chemical Society, copyright 2008.

Apart from the sensing of lectins, glycosylated fluorescent PPE polymers bearing either mannose or galactose residues and polymers without carbohydrates were also applied to the detection of bacteria. ${ }^{258}$ Then two Escherichia coli bacterial strains were used to determine if the mannosylated PPE and the non-functionalized one could interact with $E$. coli. In addition to the native strain binding mannosides, a mutated strain with no binding to mannosides was used. Clearly, PPE without mannose could not bind to either $E$. coli strains, while the mannosylated polymer could strongly bind to $E$. coli and show a fluorescent label (Figure 93). The galactosylated PPE was used as a negative control.

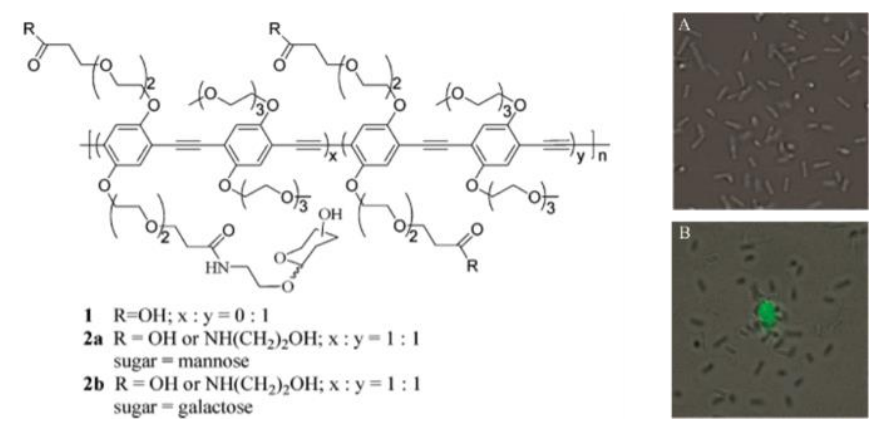

Figure 93 Structures of the glycosylated PPE fluorescent polymers, fluorescence image of bacteria E. coli and PPE 1 without mannose (A), and images of PPE $2 a$ to form fluorescent bacterial aggregate (B). Reproduced from ref. ${ }^{258}$ with permission from the American Chemical Society, copyright 2004.
Galactosylated PPEs with additional triethylammonium groups were used for targeted hepatoma cell imaging based on binding between the glycosylated PPE and HepG2 cancer cell lines as talked above, while the cancer HeLa cells were used as control. ${ }^{259}$ The three PPEs bearing different linker arms between the polymeric backbone and the ammonium groups were investigated. The results indicated that the PPE with six methylenes showed the best targeted fluorescence imaging ability for ASGPr-expressing HepG2 cells (Figure 94).

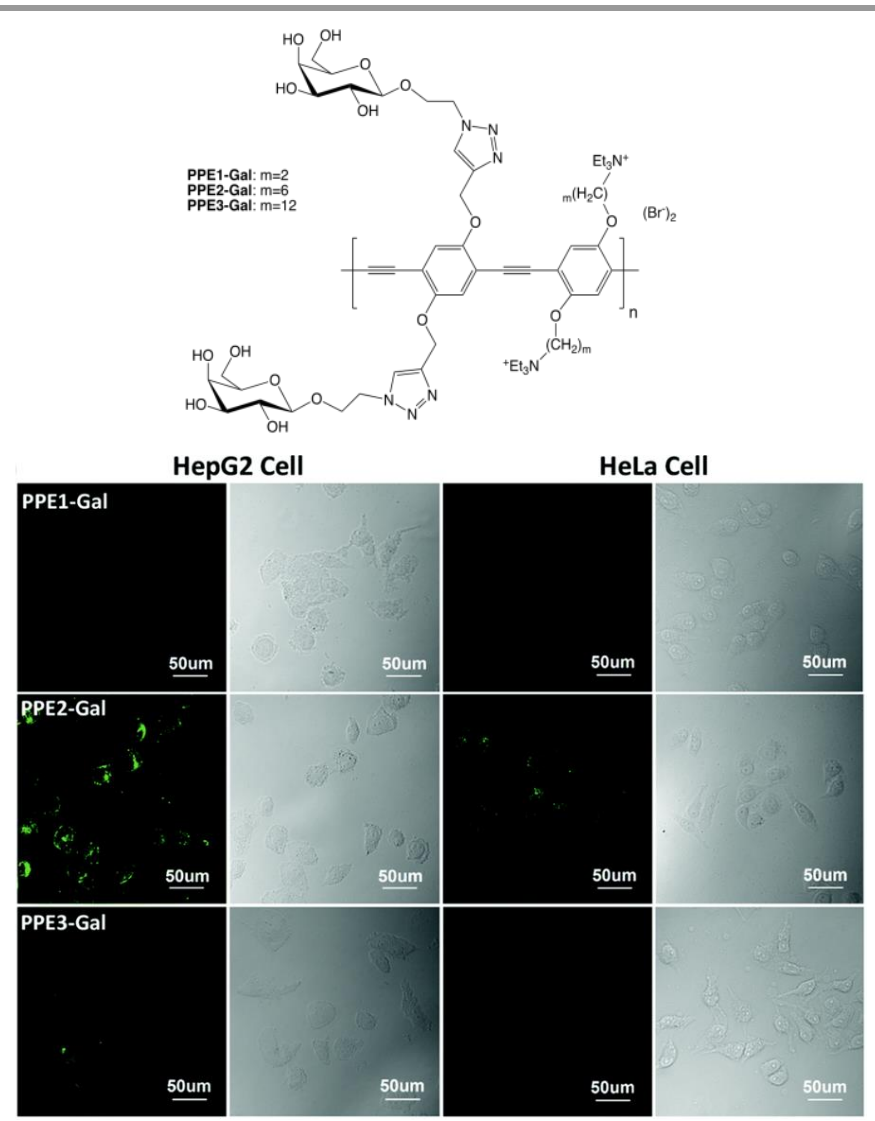

Figure 94 Structures of three galactosylated PPEs and confocal imaging of all three probes in HepG2 cells and control HeLa cells. Reproduced from ref. ${ }^{259}$ with permission from the Royal Society of Chemistry, copyright 2017.

Another mannosylated polymer was reported for the detection of ConA (Figure 95).260 The fluorescence sensing properties of this polymer toward ConA were similar to the previously discussed PPE-Man. However, the presence of ConA could actually induce a fluorescence "turn-on" and the quantification of ConA could also be realized. 


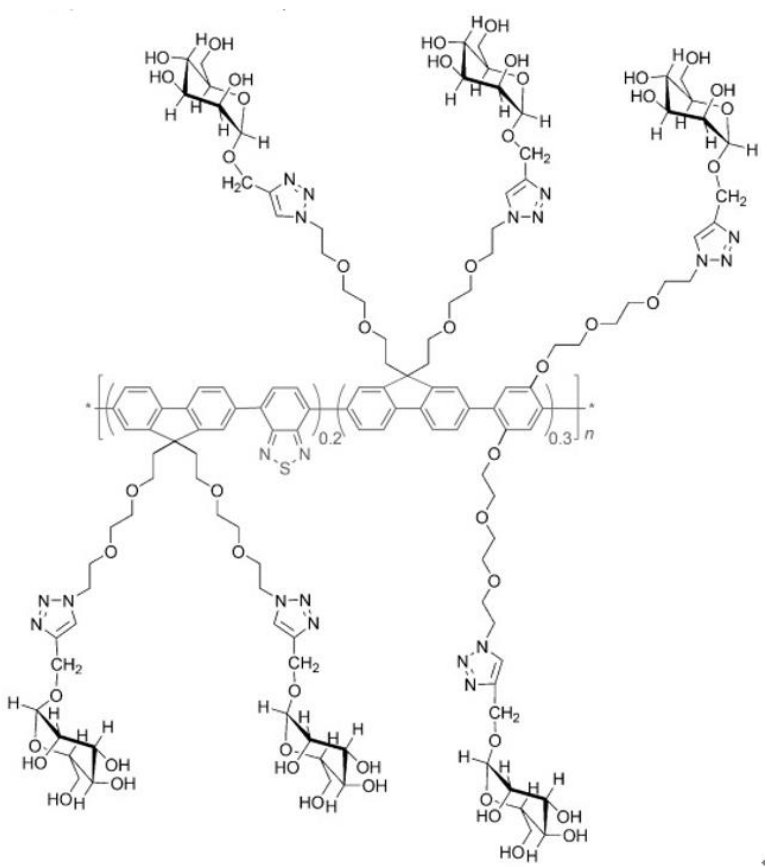

Figure 95 Structure of a Con A detecting mannosylated polymer. Reproduced from ref. ${ }^{260}$ with permission from the Wiley-VCH, copyright 2010.

The influence of the position used on the carbohydrate derivative to be conjugated to the polymeric backbone was further investigated. ${ }^{261}$ The glyco-regioisomerism effect on lectinbinding and cell-uptake pathway of galactose functionalized nanoparticles was exemplified with different linking sites (Figure 96). Both NP-6-Gal and NP-1-Man did not display any specific binding to PNA and Erythrina cristagalli agglutinin (ECA), while NP-1-Gal had strong binding to both of them. This outcome clearly indicated the glyco-regioisomersim effect in binding. However, the binding between NP-1-Gal and PNA did not lead to fluorescence quenching, which is very different from the previous reports on the interactions between lectin and carbohydrate-containing polymers (the lectin-induced aggregation would not affect the polyfluorene chain in the core of the nanoparticles). Then the binding interaction of NP1-Gal, NP-6-Gal, and NP-1-Man with human ASGPr was examined, to further study the cell-uptake pathway. The results showed that NP-1-Gal and NP-6-Gal interacted with human ASGPr while NP-1-Man did not.

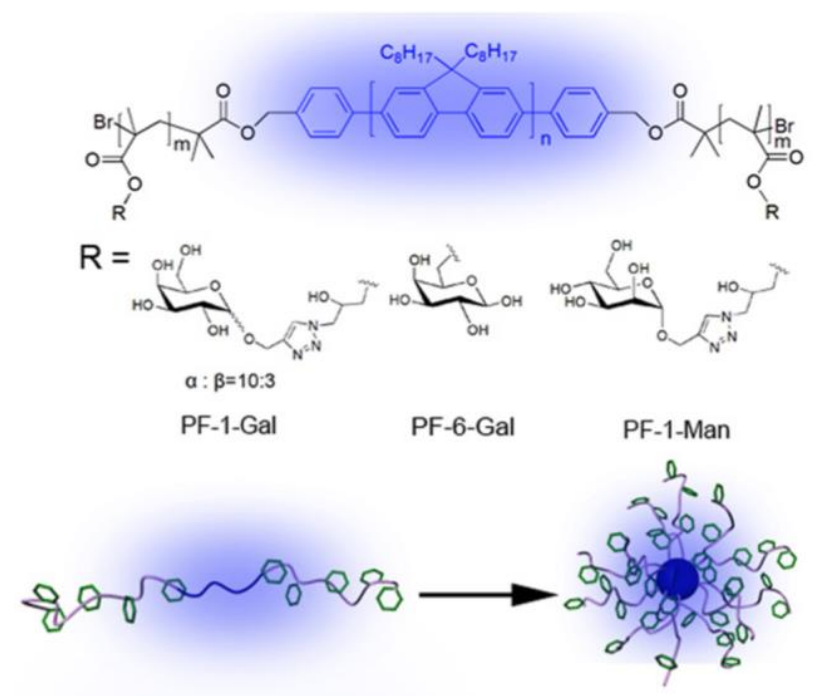

Figure 96 Structures of PF-1-Gal, PF-6-Gal, and PF-1-Man and illustration of the self-assembling process into the corresponding nanoparticles (NP-1-Gal, NP-6Gal, and NP-1-Man). Reproduced from ref. ${ }^{261}$ with permission from the American Chemical Society, copyright 2013.

Water-soluble glycosylated poly( $p$-phenylene) polymers were reported for the detection of either lectin or E. coli. ${ }^{262}$ The two polymers were mannosylated for functional experiments and glucosylated for control experiments (Figure 97). Fluorescence analysis was used to prove that the mannosylated polymer could be selectively combined with ConA since fluorescence emission quenched after addition of the lectin. Similarly, this polymer incubated with $E$. coli would lead to formation of fluorescently stained bacterial clusters. Meanwhile, the negative control glucosylated polymer did not provide any quenching in fluorescence in the presence of ConA and any killing effect upon incubation with the bacteria.
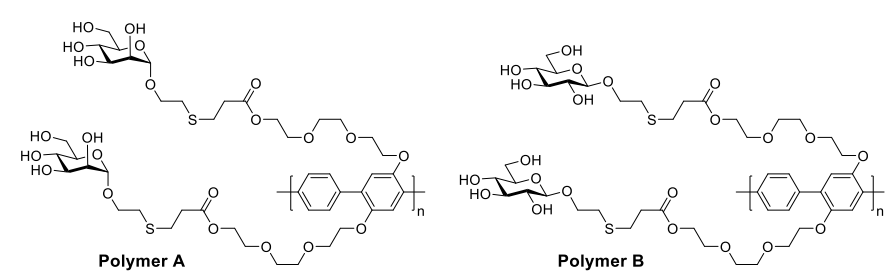

Figure 97 Structures of the mannosylated and glucosylated poly( $p$-phenylene) polymers. Reproduced from ref. ${ }^{262}$ with permission from the American Chemical Society, copyright 2006.

The application of $E$. coli detection by glycosylated polymer was also reported using water-soluble mannosylated or glucosylated polymers. ${ }^{263}$ Two strains of E. coli (ORN178 and ORN208) were chosen to conduct the evaluation and control test investigating the specific binding of the mannosylated polymer to the bacterial pili. The polymer with mannosides showed specific binding to $E$. coli and displayed clear fluorescence images (Figure 98). However, only the ORN178 strain expressing wide-type type1 pili was detected because the ORN208 strain is deficient of the FimH gene and expresses abnormal type 1 pili that do not bind to mannosides. 

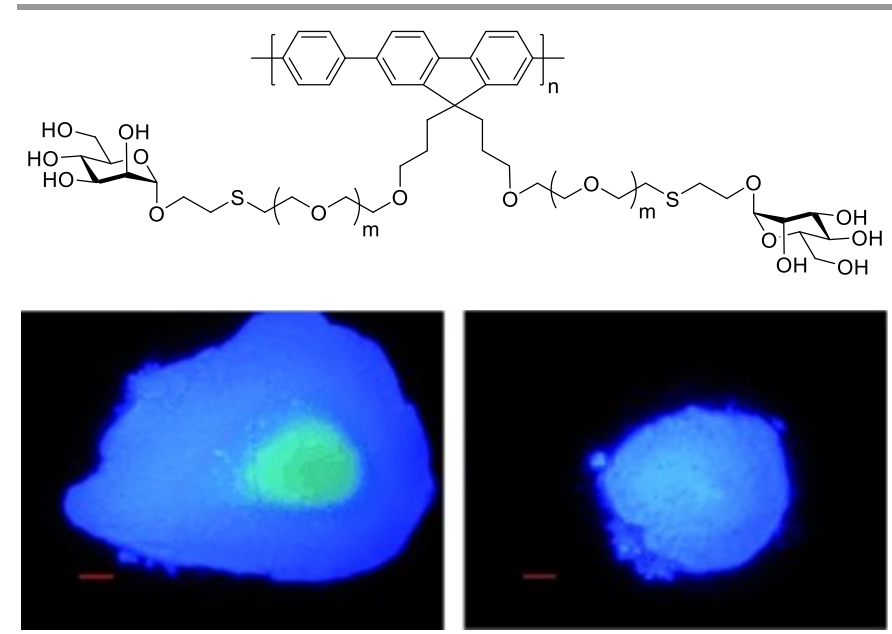

Figure 98 Structures of the mannosylated and glucosylated fluorenyl-based polymers, and fluorescent images of mannosylated glycopolymer stained $E$. coli bacteria clusters. Reproduced from ref. ${ }^{263}$ with permission from the Wiley-VCH, copyright 2009.

The pre- and post-functionalized self-assembled organic nanoparticles by $\pi$-conjugations with fluorescence emission and their application in dual targeting were studied. ${ }^{264}$ A fluorenylbenzothiadiazolyl-based polymer was synthesized and then self-assembled into a nanoparticle (pre-functionalization) (Figure 99). Then the carbohydrates were conjugated to the nanoparticle through azide-alkyne "click" reaction (postfunctionalization). A wide range of specific ligands were decorated on the particle for interactions with proteins or bacteria. The whole process can be studied by fluorescence detection because of the intrinsic fluorescence of nanoparticles, leading to selective biological targeting tools.

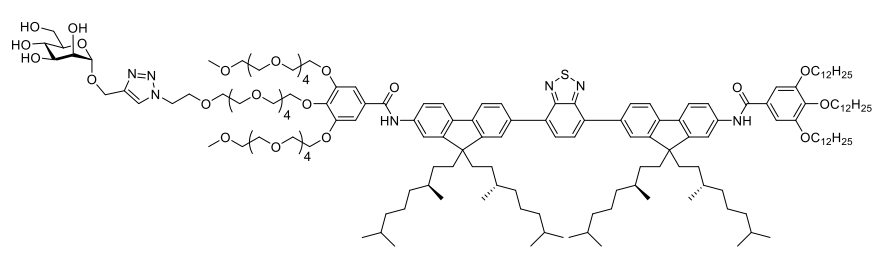

Figure 99 Structures of the mannosylated fluorenyl-benzothiadiazolyl-based nanoparticles.

\section{Glycoconjugates based on metal-centered cores for bio- medical applications}

Many luminescent transition-metal polypyridine complexes based on zinc, ruthenium(II), iridium(III), rhenium(I) and platinum(II) have been investigated for bio-imaging studies as well as medical research. ${ }^{265-271}$ Among them, metalloglycoclusters (a group of a glycocluster containing a metal core) are an interesting class of multivalent glycoconjugates due to their unique optical and photophysical properties including high emission quantum yield, photostability, long-lived excited states and intra-ligand transition emission with a large Stokes' shift (often $>5000 \mathrm{~cm}^{-1}$ ). Besides this spectroscopic properties, glycosylated metal complexes take advantage of $i$ ) the flexibility in tuning the emission wavelength by simply changing the core metal ion (e.g. lanthanides with near-infrared emission, stability and long emission lifetimes) and ii) multivalency in order to improve binding events toward a relevant biological target and cellular uptake. Moreover, these metalloglycoclusters exhibit low toxicity and good cellular internalization. ${ }^{272,273}$ All these features render them promising candidates as cell-imaging probes and biological sensors for ions, DNA, peptides and proteins. ${ }^{274-281}$ In addition, metalloglycoclusters have also explored as tools to monitor cellular glucose uptake. 274,282

Multivalent metal-based glycoclusters have been synthesized using $\mathrm{Ru}(\mathrm{II})$ complexes with three bi-pyridine moieties conjugated with lactose or maltose (Figure 100). ${ }^{283,} 284$ The complexes displayed a red-shifted emission peak in the presence of selective lectins, probably as a result of binding of the $\mathrm{Ru}$ (II) complex to a hydrophobic pocket of the lectin.

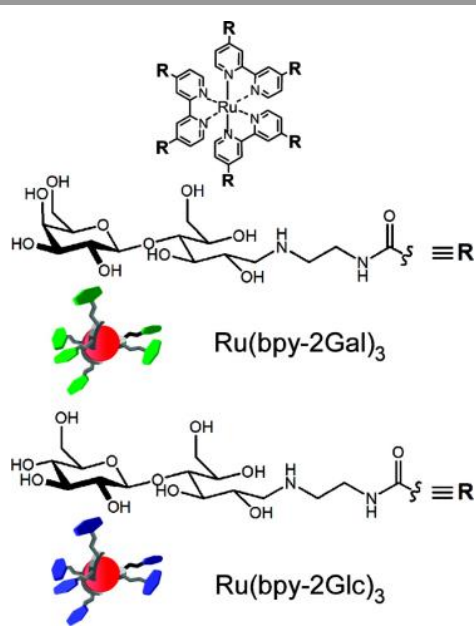

Figure 100 Structures of multivalent Ru(II)-based glycoclusters. Reproduced from ref. ${ }^{283}$ with permission from the American Chemical Society, copyright 2009.

A supramolecular strategy was described using a host-guest interaction between an adamantyl-Ru(II) complex and heptamannosylated $\beta-C D$ to provide fluorescence sensors with up to 42 carbohydrate copies. ${ }^{276}$ Fluorescence imaging of an E. coli strain (ORN178) with mannose receptors has been successfully achieved. Other $\mathrm{Cu}(\mathrm{II})$-based $^{285}$ and Fe(II)-based ${ }^{286}$ (Figure 101) multivalent glyco-metal complexes conjugated with different carbohydrates, with high binding avidity with selective lectins, have also been developed, suggesting the generality of this specific strategy. The Fe(II)-based glycoclusters were equilibrated in a dynamic combinatorial library ${ }^{287}$ of all possible exchange species in solution. Selection and strong amplification of the homo-mannosylated glycocluster was possible as the best ligands for ConA. 


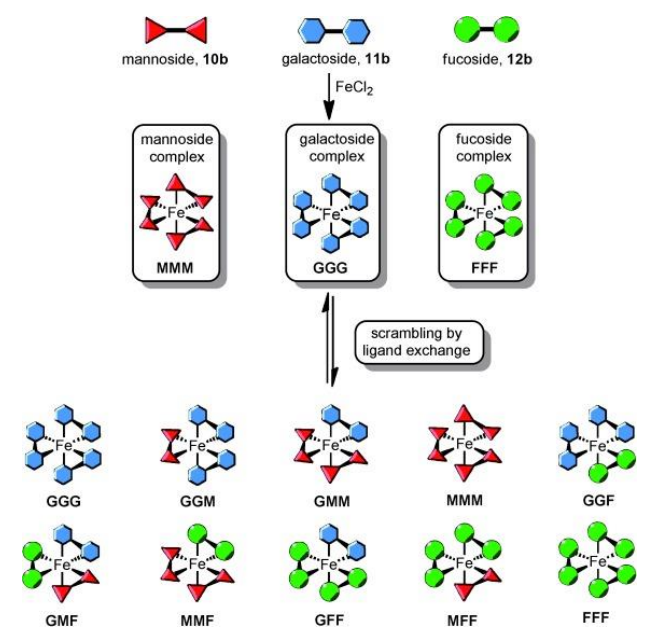

Figure $101 \mathrm{Fe}(\mathrm{II})$-based multivalent glycoclusters. Reproduced from ref. ${ }^{286}$ with permission from the Wiley-VCH, copyright 2013.

Recently, the synthesis and characterization of a novel class of metalloglycoclusters for bio-imaging application was reported. ${ }^{288}$ These glycosylated luminescent probes are based on a neutral dinuclear rhenium(I) cores bearing both 1,2-diazine ligand with different alkyl groups and two ancillary chloride ligands. ${ }^{289}$ Besides their interesting photophysical and photostability properties, $\operatorname{Re}(\mathrm{I})$ derivatives exhibit, upon excitation, a characteristic emission in the visible region, which is mainly due to the triplet metal-to-ligand charge transfer ( $\left.{ }^{3} \mathrm{MLCT}\right)$ character of the emitter. Two organometallics scaffolds have been selected for this study, depending the number of alkyl substituents: complex 1 bearing only one alkyl substituent and complex 2 with two alkyl substituents in the $\beta$ positions of the diazine ring (Figure 102). Both complexes have been synthesis according to a previously reported strategy. ${ }^{290,} 291$ Glucoside complex $\mathbf{3}$ derivate from mono-substituted complex $\mathbf{2}$ has been prepared in few steps from commercially available hex-3ynol and penta-O-acetyl-D-glucose. However, the poor overall yield (mainly owing to the last two steps) makes the strategy not suitable for large-scale synthesis. Consequently, a neoglycorandomization procedure, ${ }^{292,} 293$ involving an $\mathrm{N}$ methylamino-oxy linker and an unprotected reducing sugar, has been developed to prepare complexes $\mathbf{4}$ and $\mathbf{5}$ (consisting of glucoside and maltoside derivative, respectively). All the complexes 1-5 have a broad absorption band with the maxima in the range 347-359 $\mathrm{nm}$ and an emission in the yellow-orange region of the visible spectrum. Interestingly, mono-substituted complexes 2-3 and di-substituted complexes 1, 4-5 display remarkably different absorption and emission properties, which have highlighted the effect of the substitution of the diazine. However, conjugation of carbohydrate to the dinuclear rhenium derivative has no influence on the absorption/emission properties of the resulting glycoconjugate. In contrast, glycosylated complexes 4-5 have showed a higher quantum yield as well as longer luminescent lifetimes than the non-glycosylated parental complex 1, mainly due to aggregate formation as confirmed by NMR spectroscopy. Cellular internalization experiments have been performed on the human cervical carcinoma (HeLa) cell line. All the complexes 3-5 were internalized by living HeLa cell line and accumulated in the cytoplasm, while no migration in the nuclear region occur even at high concentration of luminescent probe. Co-staining experiments and cellular viability studies have confirmed that complexes 3-5 have a high degree of selectivity and a low cytotoxicity, an appropriate feature for bio-imaging application.

$$
\text { (n) }
$$

Figure 102 Structures of dinuclear Re parental complexes 1-2 and glycosylated Re complexes 3-5.

More recently, the same group has reported the synthesis of multivalent metalloglycoclusters bearing three copies of carbohydrates based on the above-mentioned dinuclear rhenium complex. ${ }^{294}$ These Re(I)-glycodendrimers were prepared by CuAAc starting from a trivalent alkyne scaffold with the 2(2-azidoethoxy)ethyl- $\beta$-D-galactopyranoside, 2-(2-azidoethoxy) ethyl- $\beta$-D-glucopyranoside or 2-(2-azidoethoxy)ethyl- $\alpha$-Dmannopyranoside. A subsequent condensation reaction with rhenium complex afforded the glycoconjugate luminescent probes depicted (Figure 103).288, 295 Trivalent gluco-conjugate displays an absorption band with a maximum at $323 \mathrm{~nm}$ and an emission wavelength centred around $580 \mathrm{~nm}$, but a poor luminescent quantum yield in aqueous buffer compared to monovalent glyco-Re glycoconjugates. In addition, the trivalent gluco-conjugate was unable to be internalized by living HeLa cells line as opposed for its monovalent counterpart. Both turbidimetric assay and luminescence measurements have been performed with the $\operatorname{Re}(\mathrm{l})$-glycodendrimers in order to sense the presence of two well-known lectins: ConA and PNA. As expected glycoconjugate bearing mannose was able to bind ConA. However, this binding induced a protein agglutination, which has been correlated with an enhancement of emission intensity due to AIE phenomenon. In contrast, only a modest enhancement has been observed using glycoconjugate bearing glucose (a known ligand for ConA) and no binding event occurs using galactose residue. Similarly, the intensity of the emission band centred at $580 \mathrm{~nm}$ for the metalloglycocluster containing galactose residue has increased upon the addition of the galactose-binding lectin PNA. Moreover, while treatment with nonspecific protein such as BSA have had no impact, addition of the monovalent ligand of each lectin (i.e. $\alpha$-D-ManOMe for ConA and $\beta$-D-GalOMe for PNA) led to significant decreasing of the fluorescence intensity emission due to protein desagglutination. Both experiments have confirmed the specificity of the interaction between glyco-probes and their biological targets, which is an appropriate feature for further sensing and imaging applications. 


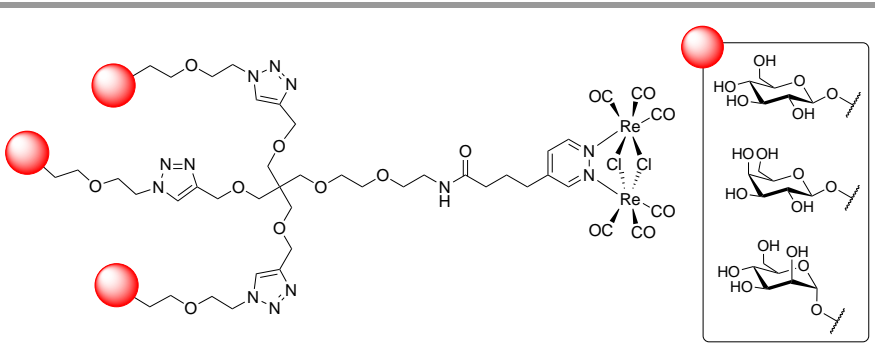

Figure 103 Structures of multivalent rhenium complex glycoconjugates.

\section{Nanomaterial-based glycoconjugates for biomedical appli- cations}

With their unique optical properties, great degree of functionality, biocompatibility, low toxicity and high valency, nanoparticles (NPs) and quantum dots (QDs) conjugated with biomolecules have been used in sensing, imaging, targeting, delivery, diagnostics and therapy. ${ }^{296-298}$ The association with carbohydrates and the intrinsic photoluminescence of most nanoparticles provide tools to explore carbohydrate-protein interactions, ${ }^{299,} 300$ since a physico-chemical perturbation on the biosensor (typically a bioreceptor probe conjugated to a transducer) might be converted into a measurable optical or electrical signal. For instance, metal nanoparticles $(\mathrm{Au}, \mathrm{Ag}, \mathrm{Pt}, \mathrm{Cu})$ composed just of a hundred of atoms exhibit intense luminescence in a broad spectral range (from UV-vis to near infrared). ${ }^{301}$ The presentation of multiple ligands onto the surface of NPs allows multivalent cooperative interactions to occur between glyconanoparticles (GNPs) and target proteins, which enhanced affinity and avidity of the glycoconjugate-protein interaction.

The synthesis of fluorescent mannnoside conjugated $\mathrm{Au}$ nanoparticles (Man-AuNPs) for the detection of vegetables lectins and bacteria has been reported (Figure 104). ${ }^{302}$ The sizes of the Man-AuNPs (from 2.9 to $1.5 \mathrm{~nm}$ ) were controlled by the Man-linker$\mathrm{SH} /$ AuNPs ratios. Although all the Man-AuNPs exhibited absorption spectra at a wavelength of $375 \mathrm{~nm}$, the sizes of the NPs have a high impact on the fluorescence emission wavelengths of the resulting GNPs (618-525 nm). First, fluorescent Man-AuNPs were used for the detection of ConA. For this purpose, various concentrations of ConA (0-1000 $\mathrm{nM}$ ) were incubated with a solution containing a fixed concentration of Man-AuNP (10 nM). After centrifugation of the reaction mixture and fluorescence measurement of the supernatant, a curve (plots of the fluorescence intensity of ManAuNPs at $545 \mathrm{~nm}$ versus the concentration of ConA) with characteristic trend has been obtained. At low concentration of ConA $(<250 \mathrm{nM})$, the lectin minimized the interaction of the ManAuNPs with potential quenchers present in solution resulting in increasing fluorescence intensity. However, when the concentration of ConA was more than $250 \mathrm{nM}$, the lectin induced aggregation of the Man-AuNPs, thereby decreasing their fluorescence. This aggregation is due to multivalent interactions between the GNPs and ConA. To improve the sensitivity, a NP-assisted protein enrichment method was employed. ${ }^{303,304}$ Green pellets obtained from precipitation after centrifugation were re-suspended in an appropriate buffer and an excess of mannose was added. After equilibration, a recovery of the fluorescence signal was observed in the supernatant, then an obvious correlation between the fluorescence intensity and the initial concentration of ConA could be established. Mannose and glucose were able to inhibit the ConAinduced aggregation of the GNPs by competing with the Man-
AuNPs as ligands of ConA. Even in the presence of 10,000 -fold higher concentration of BSA, the GNPs were able to detect ConA. Complementary assays were realized using the $E$. coli for which a minimal concentration was required to trigger the fluorescence intensity decrease due to aggregation and then increase with the addition of free mannose, as described for ConA.

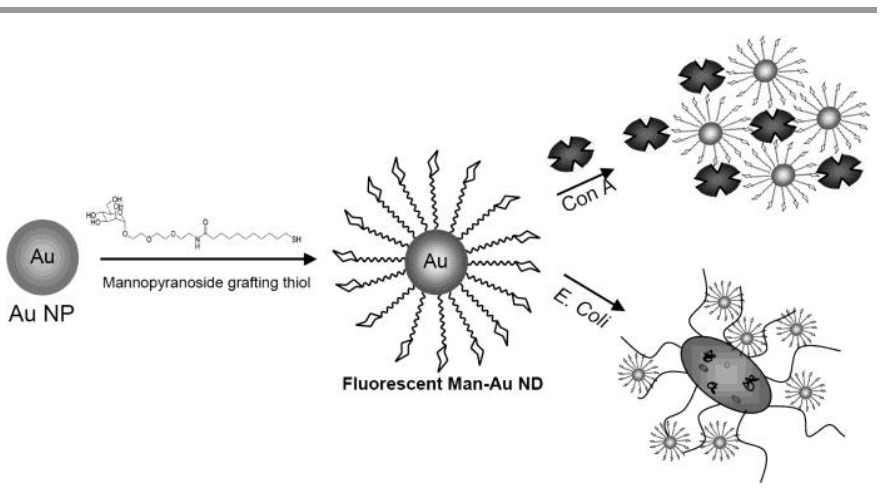

Figure 104 Mannosylated AuNPs and their interactions with ConA or E. coli. Reproduced from ref. ${ }^{302}$ with permission from the American Chemical Society, copyright 2009.

Quantitative analysis of the binding events was not possible in the previous study. To fill this gap, the quantitative binding affinity of the GNPs-lectin interactions based on a fluorescence competition-binding assay was designed. ${ }^{305}$ The aim was to measure the apparent dissociation constant $\left(\mathrm{K}_{\mathrm{d}}\right)$ of the carbohydrate-protein interaction. In addition, evaluations of the effect of ligands display on the GNPs, particularly the linker type, the spacer length, the ligand density and the nanoparticle size were studied (Figure 105). AuNPs of different size (7-30 $\mathrm{nm}$ ) were synthesized using distinct amount of sodium citrate. The mixed SAM approach using different ratio of PFPA-thiol (a or b) and 1-hexanethiol as a non-carbohydrate functionalized linker was applied to control the surface ligand density of the AuNPs. The apparent $K_{d}$ values of GNPs were then determined using a fluorescence competition assay with the interacting protein. In a typical experiment, GNPs of varying concentrations and a fixed concentration of a free competing carbohydrate were incubated with a fluorescently labelled lectin ConA (ConA-FITC) during $1 \mathrm{~h}$ prior to centrifugation. The unbound ConA-FITC as well as the free competing ligand-ConA complex remained in the supernatant, while free GNPs, including those bound to the lectin were found in the pellet. The apparent $K_{d}$ values were determined by measuring the fluorescence of the supernatant and computed according to the Cheng-Prusoff equation. ${ }^{306}$ With this protocol in hand, the effect of the ligand density was studied showing that the binding affinity generally increased with the ligand density. The impact of the spacer demonstrated that longer spacers led to enhanced binding affinity of GNPs with ConA. In this case, the steric hindrance was reduced because the carbohydrate was further away from the AuNP, and hence more accessible for interacting with the lectin. Finally, studies of the nanoparticle size have shown a binding affinity similar for the GNPs of 7, 14, or $22 \mathrm{~nm}$, whereas a decrease of approximately 6-fold was observed for the 30 $\mathrm{nm}$ GNPs. It was proposed that smaller GNPs yielded the highest affinity enhancement likely due to their large surface-tovolume ratio and higher mobility in solution. 


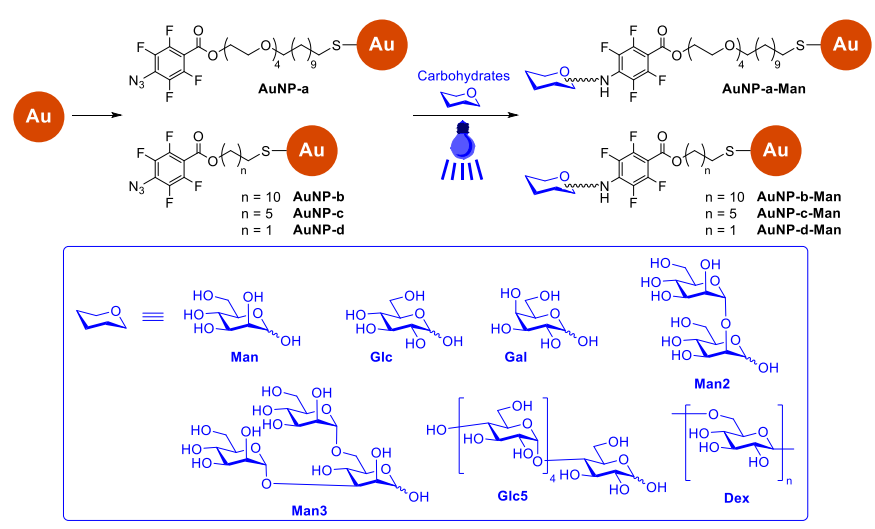

Figure 105 Structures of the glycosylated AuNPs. Reproduced from ref. ${ }^{305}$ with permission from the American Chemical Society, copyright 2010.

The synthesis of AuNPs functionalized by a mixture of zwitterionic $(\mathrm{Zw})$ and trivalent mannosylated dendrons (TriMan) was reported to determine the uptaking pathways of this nanosystem into dendritic cell (DC) (Figure 106). ${ }^{307}$ Both TriMan and Zw containing mono- or bidentate thiol groups respectively were immobilized onto the surface of the AuNP via a covalent and strong Au-S bond. Incubation of ConA with Zw-TriMan-AuNP led to a turbid solution due to aggregation and could be correlated with an increase of fluorescence at $685 \mathrm{~nm}$. This effect of aggregation-induced emission (AIE) ${ }^{308}$, 309 was likely the result of electrostatic interactions between ConA and mannose residues. In contrast, neither aggregate formation and consequently nor fluorescence variation were observed using Zw-AuNP, demonstrating the predominant role of the carbohydrate part for NPs uptaking into DCs. Additional assays by flow cytometry have demonstrated that the uptake in DCs was 3.5-fold stronger for Zw-TriMan-AuNP than for ZwAuNP, suggesting that the presence of TriMan enhanced the NP cellular accumulation. This assumption was confirmed by TEM (Transmission Electron Microscopy) showing an accumulation of Zw-TriMan-AuNP in both early and late endosomes as well as lysosomes close to the Golgi apparatus, which was in accordance with their uptake using endocytic pathways. To gain more insight into the mechanisms involved in DC uptake of Zw-TriMan-AuNP, a series of inhibitors that differentially affected the endocytic pathways were chosen and subsequently incubated with the lectin and the GNPs. The results have shown that multiple endocytic pathways were implicated, mainly via clathrin-mediated and F-actin-dependent mechanisms. In addition, mannan was selected as an inhibitor of Ctype lectin receptors such as mannose receptor (MR) and dendritic cell-specific intercellular adhesion molecule-3grabbing non-integrin (DC-SIGN). Flow cytometry measurements have shown a drastic reduction in Zw-TriMan-AuNP uptake in the presence of mannan, while no effect was observed with Zw-AuNP, demonstrating that the specific targeting of AuNPs to DCs occurs via C-type lectin receptors.

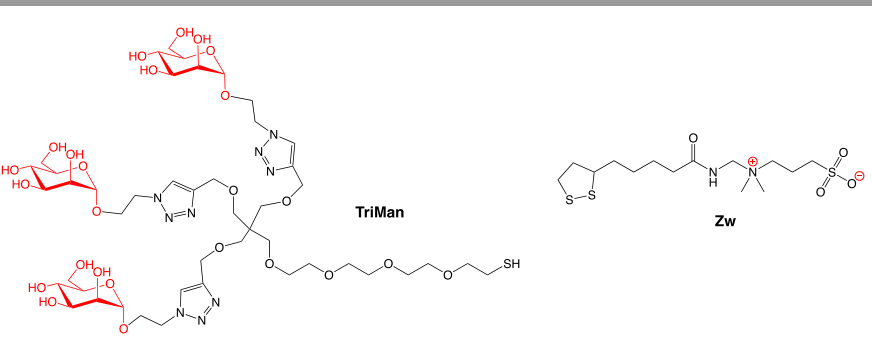

Figure 106 Mannosylated dendron (TriMan) and zwitterionic compound (Zw).

In another interesting study, the design and synthesis of a nanobiosensor was reported for the monitoring of glucose level in blood, as a clinical indicator of diabetes. ${ }^{310}$ The sensing approach was based on FRET between ConA-conjugated CdTe quantum dots (ConA-QDs) as an energy donor and $\beta$-CDs conjugated with AuNPs ( $\beta$-CD-AuNPs) as a fluorescent quencher. To this end, ConA and thiolated $\beta$-CDs ( $\beta$-SH-CDs) were conjugated with CdTe QDs and AuNPs, respectively. In absence of glucose, the specific binding of ConA with $\beta$-CDs allows NPs to be close enough of QDs so that the acceptors quench the emission of the energy donor (FRET effect on, Figure 107). However, in the presence of glucose, this monosaccharide competes with $\beta$-CDs in the binding to ConA, which displaces the $\beta$-CD-AuNPs from the nanobiosensors resulting in the fluorescence recovery of the quenched QDs (FRET effect off, Figure 107). After characterisation of both $\beta$-CD-AuNPs and ConA-QDs, the binding studies of ConA with $\beta$-CDs were performed. As expected, the fluorescence intensity of ConA-QDs decreased upon addition of $\beta$-CD-AuNPs, which confirmed the formation of the nanobiosensor ConA-QDs/ $\beta$-CD-AuNPs. Addition of glucose into the solution led to an increase in fluorescence intensity proportional to the concentration of the competing monosaccharide. Due to their high selectivity, high sensitivity and the fact that only a small amount of biological sample was required, these nanobiosensors were then applied to the detection of glucose in normal adult serum.

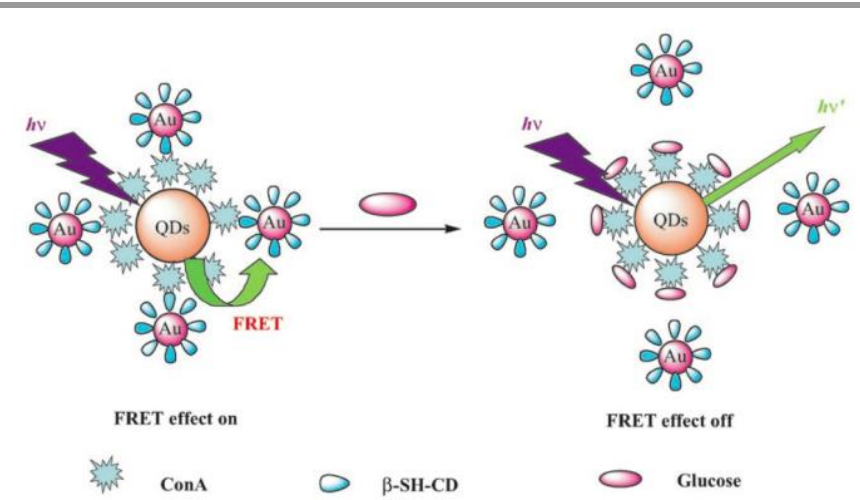

Figure 107 Mechanism proposed for the detection of glucose with the ConA$\mathrm{QDs} / \beta$-CD-AuNPs nanobiosensor. Reproduced from ref. ${ }^{310}$ with permission from the Wiley-VCH, copyright 2008.

Polyvalent mannosylated QDs were synthesized and designed to probe its interaction with dendritic cell receptors (DC-SIGN and DCSIGNR) based on FRET assays. ${ }^{311}$ Two ligands were designed (QD$\mathrm{PEG}_{3}-$ Man and QD-PEG $13-$ Man, Figure 108A) with a DHLA moiety for conjugation to QDs, two PEG linkers ( $n=3$ or 13 ) for reducing nonspecific absorption and enhancing both stability and biocompatibility of the resulting conjugate and a mannoside residue for specific 
protein recognition. An additional DHLA-zwitterion (DHLA-Zw, Figure 108) ligand was designed to study ligand density effect and controlling the spacing between each QD-PEG - Man (Figure 108CD). Subsequently, both DC-SIGN and DC-SIGNR were labelled with Atto-594 dye to investigate their multivalent binding with QD-PEG ${ }_{n}$ Man. First, the binding of the labelled DC-SIGN to the QDs probe was studied (Figure 108E). The results have shown that a reduced QD fluorescence could be correlated with an enhanced Atto-594 FRET signal, which was fully in accordance with a FRET mechanism. Interestingly, the FRET effect was more pronounced for DC-SIGN binding to $\mathrm{QD}-\mathrm{PEG}_{3}-\mathrm{Man}$ over $\mathrm{QD}-\mathrm{PEG}_{13}-\mathrm{Man}$, suggesting more efficient FRET mechanism in the former pair. Indeed, ITC studies have demonstrated that the QDs interaction with DC-SIGN was enthalpy driven, in which QD-PEG - Man has shown both a greater negative enthalpy and entropy changes than the corresponding QD$\mathrm{PEG}_{13}-\mathrm{Man}$. In addition, the FRET signal was diminished in the absence of $\mathrm{Ca}^{2+}$ and inhibited by free mannose, which is consistent with $\mathrm{Ca}^{2+}$-dependent DC-SIGN mannose binding events. Diminishing the mannose density by diluting the QD surface with DHLA-Zw was found to drastically reduce their affinity with DC-SIGN.

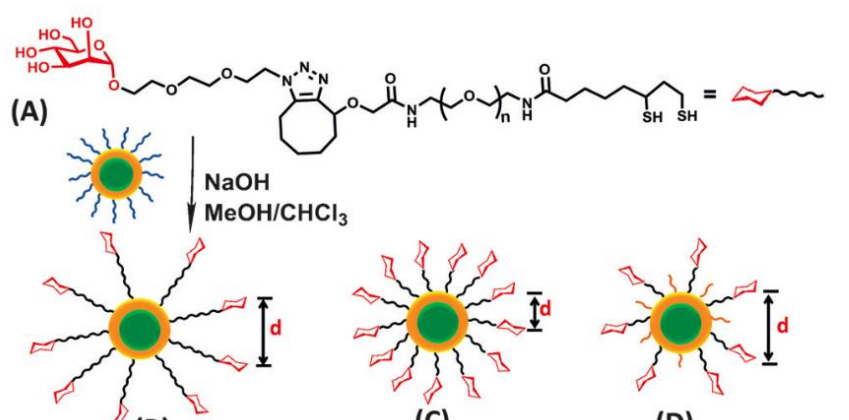

(B)

(C)

(D)

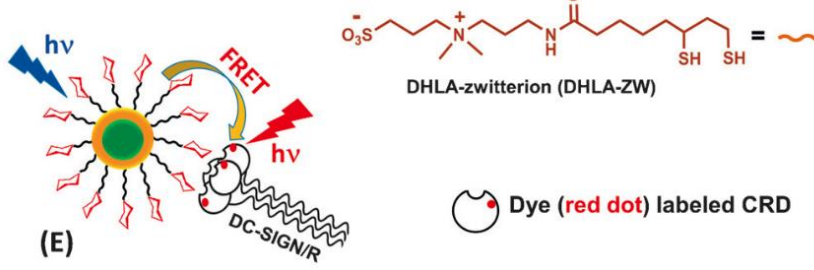

Figure 108 Preparation of QD-PEG $n$-Man for the detection of DC-SIGN. Reproduced from ref. ${ }^{311}$ with permission from the Wiley- $\mathrm{VCH}$, copyright 2016.

Surprisingly, although DC-SIGN and DC-SIGNR are two closely related tetrameric receptors sharing $77 \%$ identical sequence, binding of labelled DC-SIGNR to the QDs yielded only very weak FRET signals, which implies an inability to form effective multivalent binding. To explain why the polyvalent QD binds to DC-SIGN but very poorly or not to DC-SIGNR, it was proposed that the carbohydraterecognition domains (CRDs) were facing upwardly along the coiledcoil axes in DC-SIGN (Figure 109B), while they were found sideways in DC-SIGNR (Figure 109C).

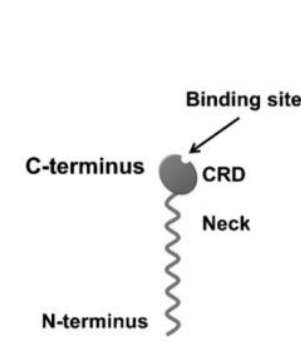

(A)

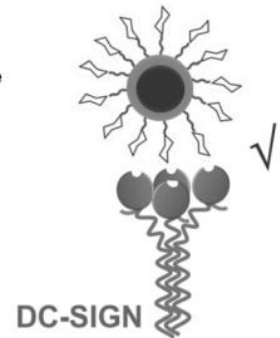

(B)

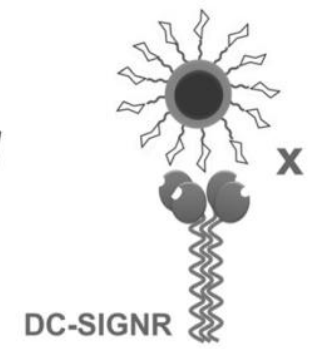

(C)
Figure 109 Mechanism proposed for the selectivity between DC-SIGN and DCSIGNR. Reproduced from ref. ${ }^{311}$ with permission from the Wiley-VCH, copyright 2016.

Aggregation-induced emissive nanoparticles were designed based on glycosylated thiophthalonitriles (Figure 110). ${ }^{312}$ The amphiphilic compounds could self-assemble into nanoparticles in water with a median size of $16 \mathrm{~nm}$ and an AIE effect. The mannoside residues could allow interactions with ConA selectively and provide a useful tool for selective bacterial aggregation detection of $E$. coli strain ORN178 over ORN208 through their fluorescence properties.<smiles>CCCOC(C)(C)CCn1cc(COC2O[C@H](CO)[C@@H](O)C(O)[C@H]2O)nn1</smiles>
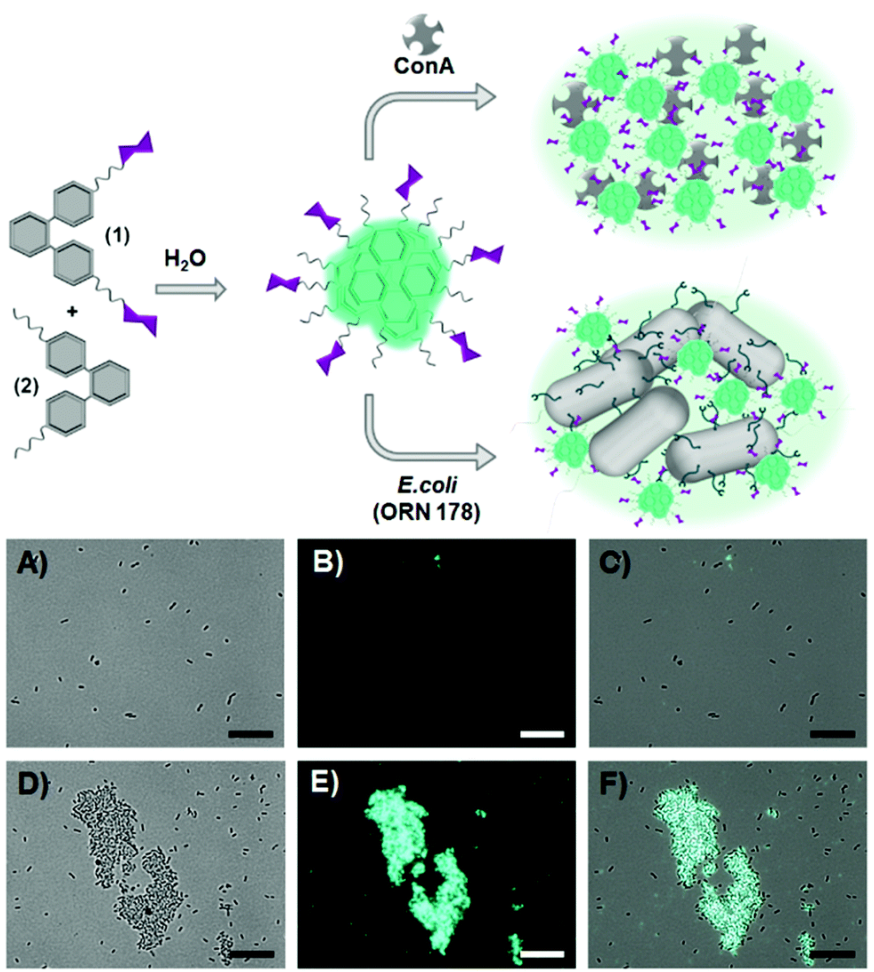

Figure $\mathbf{1 1 0}$ Structures of glycosylated thiophthalonitriles and representation of the aggregation of lectins and bacteria in the presence of nanoparticles. Bacterial aggregation imaged by bright field $(A, D)$ and fluorescence microscopy $(B, E)$ 
images. (C,F) overlay. (A-C) ORN 208 and (D-F) ORN 178. Scale bar $=20 \mu \mathrm{m}$. Reproduced from ref. ${ }^{312}$ with permission from the Royal Society of Chemistry, copyright 2016.

\section{Conclusion and outlook}

The design and applications of fluorescent glycoconjugates requires the collaborative work of researcher in physics, chemistry, biochemistry, biology, medical sciences and chemical engineering. Such research studies are therefore highly dependent on the synergistic combination of several scientific backgrounds. The initial properties of the designed glycoprobe can sometimes appear as not acting as expected, but a careful analysis of the situation and broader knowledge can typically find another positive type of application for sensing.

The designed glyco-probes must have specific properties such as emission at the NIR region (>700 $\mathrm{nm}$ ) to minimalize biological background fluorescence. The chemical conjugations are usually performed through robust and high yielding reaction such as CuAAC or amide bond formation. The conjugation of carbohydrate enables the water solubility properties and realizes the targeting of pathogens (bacteria or viruses) or cancer cells.

A compilation of the advantages and drawbacks for selected fluorescent glycoconjugates (Table 1 ) provides an overview of the different properties obtained for such probes. Bacteria can be readily detected using a fluorescent core with carbohydrates conjugates in a multivalent fashion using a fluorescent core (Entries 1,2), a fluorescent polymer (Entry 13) or a glyconanoparticle (Entry 15). The fine tuning of the fluorescence emission of these conjugates was critical for the application pursued. Detection of ions in cells appeared also as typical applications (Entries 3,6) although some studies only reported data for detection in solution (Entries 11,12). This appears as a limitation such as crossing the cellular membrane to be internalized and find its target analyte. Other probes have been applied to the detection of lectins (Entries 7,10,14) sometimes within cells. Miscellaneous applications to imaging of liposomes (Entry 4), gelation properties (Entry 5), photodynamic therapy (Entry 8) or imaging of cancer cells (Entry 9) can also be typically obtained from rather simple molecular design.

AIE is a major property used in several glyco-probe design. Meanwhile, ACQ might also prevent the proper applications of such probes. The aqueous biological media are highly adapted to AIE through aggregation of partially water soluble glycoprobes leading to "off-on" fluorescence sensors. Ratiometric detection can be readily achieved for several glyco-probes allowing quantitative detection of analytes.

Biomedical applications such as PDT/PTT, targeted drug delivery or diagnostics are common using glyco-probes, and very encouraging results have been obtained in that area. Nevertheless, little attention has been devoted towards the comprehensive evaluation of toxicity and biocompatibility of the glyco-probes, maybe because the quantity that would be used would be very minimal and below toxicity thresholds. Similarly, radio-labelled probes for $\mathrm{MRI}$ or other imaging systems are toxic, but they are with a very short decay time. Hence, little to no negative impact would be generated for the patients. Another further development in this context of fluorescent glycoconjugates would be the design and development of theranostic applications in which the probe is used to image and detect a specific cell or pathogen. Then, activation (typically through a laser) would able to trigger ROS to kill the target cells or pathogens in a therapeutic approach.

\begin{tabular}{|c|c|c|c|c|}
\hline Entry & Glycoconjugates and applications & Advantages & Drawbacks & Ref. \\
\hline 1 & Detection of cholera tox & $\begin{array}{l}\text { - Sensitive "turn-on" detection } \\
\text { enabling imaging } \\
\text { - Water solubility } \\
\text {-Easy "click" conjugation }\end{array}$ & $\begin{array}{l}\text { - Short emission wavelength, causing false- } \\
\text { positive signal in biological samples } \\
\text { - Use of toxic copper catalyst in "click" } \\
\text { reaction }\end{array}$ & 41 \\
\hline 2 & Detection and staining of Escher & $\begin{array}{l}\text { - One-pot preparation of the P3 } \\
\text { polymer } \\
\text { - Good biocompatibility } \\
\text { - Strong and selective affinity to } E \text {. } \\
\text { coli } \mathrm{DH} 5 \alpha\end{array}$ & $\begin{array}{l}\text { - Use of toxic copper catalyst in "click" } \\
\text { reaction }\end{array}$ & 65 \\
\hline 3 & Ho $\underbrace{\mathrm{OH}}_{\mathrm{OH}}$ & $\begin{array}{l}\text { - High sensitivity to } \mathrm{Zn}(\mathrm{II}) \\
\text { - Good solubility, working pH range } \\
\text { and targeting ability with zinc ions } \\
\text { - Low cytotoxicity }\end{array}$ & $\begin{array}{l}\text { - Non-specific cellular internalization with } \\
\mathrm{Zn}(\mathrm{II})\end{array}$ & 94 \\
\hline
\end{tabular}




\begin{tabular}{|c|c|c|c|c|}
\hline 4 & Imaging within liposome & $\begin{array}{l}\text { - Strong targeting effect and self- } \\
\text { assembling to enable the probe to } \\
\text { specifically image the liposomes } \\
\text { based on an emission color change } \\
\text { - Further application in glycan-lectin } \\
\text { study and bacterial detection }\end{array}$ & $\begin{array}{l}\text { - Synthesis of the probe requires microwaves } \\
\text { irradiation, heating at } 100^{\circ} \mathrm{C} \text { for } 2 \text { days } \\
\text { would not trigger the reaction }\end{array}$ & 109 \\
\hline 5 & Gelation and biological antioxidation & $\begin{array}{l}\text { - Excellent free radical scavenging } \\
\text { compared } \\
\text { - Good bioavailability and biocom- } \\
\text { patibility } \\
\text { - Low cytotoxicity }\end{array}$ & $\begin{array}{l}\text { - Evaluation only in solution, no report of the } \\
\text { activities in vivo or in tissues }\end{array}$ & 128 \\
\hline 6 & Imaging of $\mathrm{Hg}(\mathrm{II})$ in HepG2 cells & $\begin{array}{l}\text { - in situ imaging of endogenous } \\
\mathrm{Hg}(\mathrm{II}) \\
\text { - Low toxicity towards HepG2 cells } \\
\text { - Two galactose residues for a } \\
\text { better targeting effect }\end{array}$ & $\begin{array}{l}\text { - Use of toxic copper catalyst in "click" } \\
\text { reaction }\end{array}$ & 139 \\
\hline 7 & Detection of $\beta$-amyloid and discrimination of lectins & $\begin{array}{l}\text { - Sensitive detection of } A \beta \text { peptides } \\
\text { as well as their fibrils } \\
\text { - Lectin detection with good bio- } \\
\text { specificity }\end{array}$ & $\begin{array}{l}\text { - Use of toxic copper catalyst in "click" } \\
\text { reaction }\end{array}$ & 155 \\
\hline 8 & Potential PDT agents & $\begin{array}{l}\text { - Maximum absorption }>680 \mathrm{~nm} \\
\text { - Good water solubility } \\
\text { - Good stability for use as photosen- } \\
\text { sitizers }\end{array}$ & $\begin{array}{l}\text { - Application of PDT was just expected, no } \\
\text { assay was reported } \\
\text { - Complex multi-step synthesis }\end{array}$ & 179 \\
\hline 9 & Imaging of MCF-7 cells & $\begin{array}{l}\text { - Self-assembly into particles with } \\
\text { good fluorescence quantum yield } \\
\text { - A reactive amine group on each } \\
\text { glucopyranose for further modifica- } \\
\text { tions }\end{array}$ & $\begin{array}{l}\text { - Better uptake inside MCF-7 cancer cells } \\
\text { upon conjugation with folic acid (FA), prov- } \\
\text { ing FA was better in targeting MCF-7 }\end{array}$ & 191 \\
\hline 10 & $\begin{array}{l}\text { Detection of lectin (PNA) and two-photon imaging of } \\
\mathrm{HepG} 2 \text { cells }\end{array}$ & $\begin{array}{l}\text { - High selectivity to image HepG2 } \\
\text { cells }\end{array}$ & $\begin{array}{l}\text { - Use of toxic copper catalyst in "click" } \\
\text { reaction }\end{array}$ & 192 \\
\hline 11 & Detection of $\mathrm{Ni}(\mathrm{II})$ & $\begin{array}{l}\text { - Unexpected optical properties } \\
\text { - Selective sensing of } \mathrm{Ni}(\mathrm{II}) \text { over } \\
\text { other metal ions } \\
\text { - Another bioactivity as inhibitor of } \\
\text { PTP1B (type } 2 \text { diabetes) }\end{array}$ & - Detection of ions only in solution & 209 \\
\hline
\end{tabular}




\begin{tabular}{|c|c|c|c|c|}
\hline 12 & Detection of $\mathrm{Ni(II)}$ & $\begin{array}{l}\text { - Highly sensitive to } \mathrm{Ni}(\mathrm{II}) \\
\text { - The use of thiadiazole as a metal } \\
\text { binding site with high binding } \\
\text { affinity }\end{array}$ & $\begin{array}{l}\text { - Quenching signal with other ions like } \mathrm{Co}(\mathrm{II}) \text {, } \\
\mathrm{Cu}(\mathrm{II}), \mathrm{Hg}(\mathrm{II}) \\
\text { - Evaluation only in solution, no report of the } \\
\text { activities in vivo or in tissues }\end{array}$ & 228 \\
\hline 13 & Strain-specific staining and detection of Escherichia & $\begin{array}{l}\text { - Good water solubility } \\
\text { - Specific affinity to } E \text {. coli strain } \\
\text { ORN178 with FimH proteins, no } \\
\text { affinity to other strains without } \\
\text { FimH }\end{array}$ & - A multistep and complex synthetic route & 263 \\
\hline 14 & Detection of lectins & $\begin{array}{l}\text { - The metal center has intrinsic } \\
\text { properties that can be further } \\
\text { utilized }\end{array}$ & N/A & 283 \\
\hline 15 & $\mathrm{Au} \stackrel{\text { Detection of ConA and Escherichia coli }}{\longrightarrow}$ & $\begin{array}{l}\text { - An easy preparation and purifica- } \\
\text { tion method } \\
\text { - Good water solubility } \\
\text { - Higher fluorescence quantum yield } \\
\text { than typical Au nanoclusters }\end{array}$ & N/A & 302 \\
\hline
\end{tabular}

130 Meilong Rd., Shanghai 200237, PR China. Email: mrs guorongchen@ecust.edu.cn (G.-R. C.), xphe@ecust.edu.cn (X.-P.H.)

\section{Acknowledgements}

The authors thank the Université Claude Bernard Lyon 1 and the CNRS for financial support. S. V. and B. T. acknowledge support from the ANR project Bacteri'eau (ANR-16-CE04-001402). K.-C. Y., X.-L. H., G.-R. C. and X.-P. H. wish to thank the National Natural Science Foundation of China (Nos. 21788102, 91853201, 21776078 and 21722801), the Shanghai Municipal Science and Technology Major Project (No. 2018SHZDZX03) and the International Cooperation Program of Shanghai Science and Technology Committee (No. 17520750100).

\section{Notes and references}

a Institut de Chimie et Biochimie Moléculaires et Supramoléculaires, Laboratoire de Chimie Organique 2-Glycochimie, UMR 5246, CNRS and Université Claude Bernard Lyon 1, Université de Lyon, 1 Rue Victor Grignard, F-69622 Villeurbanne, France. Email: sebastien.vidal@univlyon1.fr

${ }^{b}$ Key Laboratory for Advanced Materials and Joint International Research Laboratory of Precision Chemistry and Molecular Engineering, Feringa Nobel Prize Scientist Joint Research Center, School of Chemistry and Molecular Engineering, East China University of Science and Technology,
${ }^{1}$ These authors contributed equally to this work.

1. H.-J. Gabius, The sugar code, Wiley-VCH Weinheim, 2009.

2. Y. C. Lee and R. T. Lee, Acc. Chem. Res., 2002, 28, 321-327.

3. J. J. Lundquist and E. J. Toone, Chem. Rev., 2002, 102, 555-578.

4. J. L. Jimenez Blanco, C. Ortiz Mellet and J. M. Garcia Fernandez, Chem. Soc. Rev., 2013, 42, 4518-4531.

5. Y. Chen, A. Star and S. Vidal, Chem. Soc. Rev., 2013, 42, 4532-4542.

6. K. Hatano, K. Matsuoka and D. Terunuma, Chem. Soc. Rev., 2013, 42, 4574-4598.

7. M. C. Galan, P. Dumy and O. Renaudet, Chem. Soc. Rev., 2013, 42, 4599-4612.

8. F. Sansone and A. Casnati, Chem. Soc. Rev., 2013, 42, 4623-4639.

9. N. Jayaraman, K. Maiti and K. Naresh, Chem. Soc. Rev., 2013, 42, 4640-4656.

10. Y. M. Chabre and R. Roy, Chem. Soc. Rev., 2013, 42, 4657-4708.

11. A. Bernardi, J. Jimenez-Barbero, A. Casnati, C. De Castro, T. Darbre, F. Fieschi, J. Finne, H. Funken, K. E. Jaeger, M. Lahmann, T. K. Lindhorst, M. Marradi, P. Messner, A. Molinaro, P. V. Murphy, C. Nativi, S. Oscarson, S. Penades, F. Peri, R. J. Pieters, O. Renaudet, J. L. Reymond, B. Richichi, J. Rojo, F. Sansone, C. Schaffer, W. B. Turnbull, T. Velasco-Torrijos, S. Vidal, S. Vincent, T. Wennekes, H. Zuilhof and A. Imberty, Chem. Soc. Rev., 2013, 42, 4709-4727.

12. A. Martinez, C. Ortiz Mellet and J. M. Garcia Fernandez, Chem. Soc. Rev., 2013, 42, 4746-4773. 
13. M. Marradi, F. Chiodo, I. Garcia and S. Penades, Chem. Soc. Rev., 2013, 42, 4728-4745.

14. S. Cecioni, A. Imberty and S. Vidal, Chem. Rev., 2015, 115, 525-561.

15. T. R. Branson and W. B. Turnbull, Chem. Soc. Rev., 2013, 42, 46134622.

16. J. L. Reymond, M. Bergmann and T. Darbre, Chem. Soc. Rev., 2013, 42, 4814-4822.

17. Z. Guo, S. Park, J. Yoon and I. Shin, Chem. Soc. Rev., 2014, 43, 16-29.

18. J. Yao, M. Yang and Y. Duan, Chem. Rev., 2014, 114, 6130-6178.

19. H. Zhu, J. Fan, J. Du and X. Peng, Acc. Chem. Res., 2016, 49, 21152126.

20. H. Yan, R. S. Yalagala and F. Yan, Glycoconj. J., 2015, 32, 559-574.

21. X. P. He, Y. Zang, T. D. James, J. Li, G. R. Chen and J. Xie, Chem. Commun., 2016, 53, 82-90.

22. Y. Hong, J. W. Lam and B. Z. Tang, Chem. Soc. Rev., 2011, 40, 53615388.

23. C. Zhu, R. T. K. Kwok, J. W. Y. Lam and B. Z. Tang, ACS Appl. Bio Materials, 2018, 1, 1768-1786.

24. Y. Chen, J. W. Y. Lam, R. T. K. Kwok, B. Liu and B. Z. Tang, Mater. Horiz., 2019, 6, 428-433.

25. Y. Tang and B. Z. Tang, Principles and Applications of AggregationInduced Emission, Springer Nature 2019.

26. Y. Cai, L. Du, K. Samedov, X. Gu, F. Qi, H. H. Y. Sung, B. O. Patrick, Z. Yan, X. Jiang, H. Zhang, J. W. Y. Lam, I. D. Williams, D. Lee Phillips, A. Qin and B. Z. Tang, Chem. Sci., 2018, 9, 4662-4670.

27. Z. Yang, W. Qin, N. L. C. Leung, M. Arseneault, J. W. Y. Lam, G. Liang, H. H. Y. Sung, I. D. Williams and B. Z. Tang, J. Mater. Chem. C, 2016, 4, 99-107.

28. G. Sun, H. Zhou, Y. Liu, Y. Li, Z. Zhang, J. Mei and J. Su, ACS Appl. Mater. Interfaces, 2018, 10, $20205-20212$.

29. J. Wang, X. Yao, Y. Liu, H. Zhou, W. Chen, G. Sun, J. Su, X. Ma and H. Tian, Adv. Opt. Mater., 2018, 6, 1800074.

30. L. Shi, W. Song, C. Lian, W. Chen, J. Mei, J. Su, H. Liu and H. Tian, Adv. Opt. Mater., 2018, 6, 1800190.

31. Z. Zhang, C. L. Chen, Y. A. Chen, Y. C. Wei, J. Su, H. Tian and P. T. Chou, Angew. Chem. Int. Ed., 2018, 57, 9880-9884.

32. W. Huang, L. Sun, Z. Zheng, J. Su and H. Tian, Chem. Commun., 2015, 51, 4462-4464.

33. W. T. Dou, W. Chen, X. P. He, J. Su and H. Tian, Faraday Discuss., 2017, 196, 395-402.

34. H. Wang, Y. Li, Y. Zhang, J. Mei and J. Su, Chem. Commun., 2019, 55, 1879-1882.

35. Z. Zhang, Y. S. Wu, K. C. Tang, C. L. Chen, J. W. Ho, J. Su, H. Tian and P. T. Chou, J. Am. Chem. Soc., 2015, 137, 8509-8520.

36. W. Chen, C. L. Chen, Z. Zhang, Y. A. Chen, W. C. Chao, J. Su, H. Tian and P. T. Chou, J. Am. Chem. Soc., 2017, 139, 1636-1644.

37. H. Zhou, J. Mei, Y. A. Chen, C. L. Chen, W. Chen, Z. Zhang, J. Su, P. T. Chou and H. Tian, Small, 2016, 12, 6542-6546.

38. Y. Yuan and B. Liu, Chem. Sci., 2017, 8, 2537-2546.

39. T. Sanji, K. Shiraishi, M. Nakamura and M. Tanaka, Chem. Asian J., 2010, 5, 817-824.

40. K. Shiraishi, T. Sanji and M. Tanaka, Tetrahedron Lett., 2010, 51, 63316333.

41. X. M. Hu, Q. Chen, J. X. Wang, Q. Y. Cheng, C. G. Yan, J. Cao, Y. J. He and B. H. Han, Chem. Asian J., 2011, 6, 2376-2381.

42. T. Kato, A. Kawaguchi, K. Nagata and K. Hatanaka, Biochem. Biophys. Res. Commun., 2010, 394, 200-204.

43. M. Donnier-Marechal, S. Abdullayev, M. Bauduin, Y. Pascal, M. Q. Fu, X. P. He, E. Gillon, A. Imberty, E. Kipnis, R. Dessein and S. Vidal, Org. Biomol. Chem., 2018, 16, 8804-8809.

44. J. X. Wang, Q. Chen, N. Bian, F. Yang, J. Sun, A. D. Qi, C. G. Yan and B. H. Han, Org. Biomol. Chem., 2011, 9, 2219-2226.

45. S. Andre, S. O'Sullivan, C. Koller, P. V. Murphy and H. J. Gabius, Org. Biomol. Chem., 2015, 13, 4190-4203.

46. H. Wang, G. Liu, H. Gao and Y. Wang, Polym. Chem., 2015, 6, 47154718.

47. K. Mandal and N. R. Jana, ACS Appl. Nano Materials, 2018, 1, 3531 3540.
48. Y. Hang, X. P. He, L. Yang and J. Hua, Biosens. Bioelectron., 2015, 65, 420-426.

49. Y. Hang, X. Cai, J. Wang, T. Jiang, J. Hua and B. Liu, Sci. China Chem. 2018, 61, 898-908.

50. F. Wurthner, Chem. Commun., 2004, 1564-1579.

51. Y. Avlasevich, C. Li and K. Müllen, J. Mater. Chem., 2010, 20, 38143826.

52. X. Zhan, A. Facchetti, S. Barlow, T. J. Marks, M. A. Ratner, M. R. Wasielewski and S. R. Marder, Adv. Mater., 2011, 23, 268-284.

53. F. Wurthner and M. Stolte, Chem. Commun., 2011, 47, 5109-5115.

54. D. Ke, C. Zhan, S. Xu, X. Ding, A. Peng, J. Sun, S. He, A. D. Li and J. Yao, J. Am. Chem. Soc., 2011, 133, 11022-11025.

55. B. A. Jones, A. Facchetti, M. R. Wasielewski and T. J. Marks, J. Am. Chem. Soc., 2007, 129, 15259-15278.

56. M. Yin, J. Shen, R. Gropeanu, G. O. Pflugfelder, T. Weil and K. Mullen, Small, 2008, 4, 894-898.

57. J. Qu, C. Kohl, M. Pottek and K. Mullen, Angew. Chem. Int. Ed., 2004, 43, 1528-1531.

58. K. Peneva, G. Mihov, A. Herrmann, N. Zarrabi, M. Borsch, T. M. Duncan and K. Mullen, J. Am. Chem. Soc., 2008, 130, 5398-5399.

59. F. J. Cespedes-Guirao, A. B. Ropero, E. Font-Sanchis, A. Nadal, F. Fernandez-Lazaro and A. Sastre-Santos, Chem. Commun., 2011, 47, 8307-8309.

60. K.-R. Wang, H.-W. An, F. Qian, Y.-Q. Wang, J.-C. Zhang and X.-L. Li, RSC Adv., 2013, 3, 23190-23196.

61. K. R. Wang, Y. Q. Wang, J. Li, H. W. An, L. P. Zhang, J. C. Zhang and X. L. Li, Bioorg. Med. Chem. Lett., 2013, 23, 480-483.

62. K. R. Wang, H. W. An, R. X. Rong, Z. R. Cao and X. L. Li, Biosens. Bioelectron., 2014, 58, 27-32.

63. K. R. Wang, H. W. An, R. X. Rong, Z. R. Cao and X. L. Li, Macromol. Rapid Commun., 2014, 35, 727-734

64. Y. Liu, D. K. Ji, L. Dong, N. Galanos, Y. Zang, J. Li, S. Vidal and X. P. He, Chem. Commun., 2017, 53, 11937-11940.

65. L. Q. Xu, C. Huang, R. Wang, K.-G. Neoh, E.-T. Kang and G. D. Fu, Polymer, 2011, 52, 5764-5771.

66. M. Donnier-Marechal, N. Galanos, T. Grandjean, Y. Pascal, D. K. Ji, L. Dong, E. Gillon, X. P. He, A. Imberty, E. Kipnis, R. Dessein and S. Vidal, Org. Biomol. Chem., 2017, 15, 10037-10043.

67. K. R. Wang, H. W. An, L. Wu, J. C. Zhang and X. L. Li, Chem. Commun., 2012, 48, 5644-5646.

68. K. R. Wang, H. W. An, Y. Q. Wang, J. C. Zhang and X. L. Li, Org. Biomol. Chem., 2013, 11, 1007-1012.

69. K. R. Wang, D. Han, G. J. Cao and X. L. Li, Chem. Asian J., 2015, 10, 1204-1214

70. K.-R. Wang, D. Han, G.-J. Cao and X.-L. Li, RSC Adv., 2015, 5, 4772847731.

71. L. Liang and X. Li, J. Mol. Model., 2018, 24, 51-57.

72. K. R. Wang, Z. B. Yang and X. L. Li, Chem. Eur. J., 2015, 21, 5680-5684.

73. X. Jia, Y. Yang, Y. Xu and X. Qian, Pure Appl. Chem., 2014, 86, 12371246.

74. S. Banerjee, E. B. Veale, C. M. Phelan, S. A. Murphy, G. M. Tocci, L. J. Gillespie, D. O. Frimannsson, J. M. Kelly and T. Gunnlaugsson, Chem. Soc. Rev., 2013, 42, 1601-1618.

75. L. Ingrassia, F. Lefranc, R. Kiss and T. Mijatovic, Curr. Med. Chem., 2009, 16, 1192-1213.

76. R. Greenwald and L. Golub, Curr. Med. Chem., 2001, 8, 237-242.

77. S. Tan, H. Yin, Z. Chen, X. Qian and Y. Xu, Eur J. Med. Chem., 2013, 62, 130-138.

78. K.-B. Li, D. Zhou, X.-P. He and G.-R. Chen, Dyes Pigm., 2015, 116, $52-$ 57.

79. K.-B. Li, W.-P. Jia, D.-M. Han, D.-X. Liang, X.-P. He and G.-R. Chen, Sens. Actuators B, 2017, 246, 197-201.

80. K. B. Li, N. Li, Y. Zang, G. R. Chen, J. Li, T. D. James, X. P. He and H. Tian, Chem. Sci., 2016, 7, 6325-6329.

81. X. P. He, Y. L. Zeng, X. Y. Tang, N. Li, D. M. Zhou, G. R. Chen and H. Tian, Angew. Chem. Int. Ed., 2016, 55, 13995-13999. 
82. E. Calatrava-Perez, S. A. Bright, S. Achermann, C. Moylan, M. O. Senge, E. B. Veale, D. C. Williams, T. Gunnlaugsson and E. M. Scanlan, Chem. Commun., 2016, 52, 13086-13089.

83. H. Wang, Y. Liu, C. Xu, X. Wang, G. R. Chen, T. D. James, Y. Zang, J. Li, X. Ma and X. P. He, Chem. Commun., 2018, 54, 4037-4040.

84. X. L. Hu, Y. Zang, J. Li, G. R. Chen, T. D. James, X. P. He and H. Tian, Chem. Sci., 2016, 7, 4004-4008.

85. D. Bechet, P. Couleaud, C. Frochot, M. L. Viriot, F. Guillemin and M. Barberi-Heyob, Trends Biotechnol., 2008, 26, 612-621.

86. E. Paszko, C. Ehrhardt, M. O. Senge, D. P. Kelleher and J. V. Reynolds, Photodiagnosis Photodyn. Ther., 2011, 8, 14-29.

87. D. K. Chatterjee, L. S. Fong and Y. Zhang, Adv. Drug Deliv. Rev., 2008 , 60, 1627-1637.

88. L. Cheng, C. Wang, L. Feng, K. Yang and Z. Liu, Chem. Rev., 2014, 114 10869-10939.

89. Y. Fu, H. H. Han, J. Zhang, X. P. He, B. L. Feringa and H. Tian, J. Am. Chem. Soc., 2018, 140, 8671-8674.

90. J. Zhang, Y. Fu, H. H. Han, Y. Zang, J. Li, X. P. He, B. L. Feringa and H. Tian, Nat. Commun., 2017, 8, 987-995.

91. D. T. Shi, D. Zhou, Y. Zang, J. Li, G. R. Chen, T. D. James, X. P. He and H. Tian, Chem. Commun., 2015, 51, 3653-3655.

92. Y. Fu, J. Zhang, H. Wang, J.-L. Chen, P. Zhao, G.-R. Chen and X.-P. He, Dyes Pigm., 2016, 133, 372-379.

93. X. Chai, Y. X. Fu, T. D. James, J. Zhang, X. P. He and H. Tian, Chem. Commun., 2017, 53, 9494-9497.

94. L. Dong, Y. Zang, D. Zhou, X. P. He, G. R. Chen, T. D. James and J. Li, Chem. Commun., 2015, 51, 11852-11855.

95. N. Boens, V. Leen and W. Dehaen, Chem. Soc. Rev., 2012, 41, 11301172.

96. T. Kowada, H. Maeda and K. Kikuchi, Chem. Soc. Rev., 2015, 44, 49534972.

97. Y. Ni and J. Wu, Org. Biomol. Chem., 2014, 12, 3774-3791.

98. T. Uppal, N. V. S. D. K. Bhupathiraju and M. G. H. Vicente, Tetrahedron, 2013, 69, 4687-4693.

99. P. Compain and O. R. Martin, Iminosugars: from Synthesis to Therapeutic Applications, Wiley \& Sons, Chichester, 2007.

100. N. F. Bras, N. M. Cerqueira, M. J. Ramos and P. A. Fernandes, Expert Opin. Ther. Pat., 2014, 24, 857-874.

101. T. Wrodnigg, A. Steiner and B. Ueberbacher, Anti-Cancer Agents Med. Chem., 2008, 8, 77-85.

102. H. Moriyama, T. Tsukida, Y. Inoue, K. Yokota, K. Yoshino, H. Kondo, N. Miura and S. Nishimura, J. Med. Chem., 2004, 47, 1930-1938.

103. A. Orsato, E. Barbagallo, B. Costa, S. Olivieri, L. De Gioia, F. Nicotra and B. La Ferla, Eur. J. Org. Chem., 2011, 5012-5019.

104. C. Decroocq, F. Stauffert, O. Pamlard, F. Oulaidi, E. Gallienne, O. R. Martin, C. Guillou and P. Compain, Bioorg. Med. Chem. Lett., 2015, 25, 830-833.

105. S. G. Gouin, Chem. Eur. J., 2014, 20, 11616-11628.

106. P. Compain and A. Bodlenner, ChemBioChem, 2014, 15, 1239-1251.

107. M. L. Lepage, A. Mirloup, M. Ripoll, F. Stauffert, A. Bodlenner, R. Ziessel and P. Compain, Beilstein J. Org. Chem., 2015, 11, 659-667.

108. M. R. Martinez-Gonzalez, A. Urías-Benavides, E. Alvarado-Martínez, J. C. Lopez, A. M. Gómez, M. del Rio, I. Garcia, A. Costela, J. Bañuelos, T. Arbeloa, I. Lopez Arbeloa and E. Peña-Cabrera, Eur. J. Org. Chem., 2014, 2014, 5659-5663.

109. R. S. Yalagala, S. A. Mazinani, L. A. Maddalena, J. A. Stuart, F. Yan and H. Yan, Carbohydr. Res., 2016, 424, 15-20.

110. Q. Zhang, Y. Cai, Q. Y. Li, L. N. Hao, Z. Ma, X. J. Wang and J. Yin, Chem. Eur. J., 2017, 23, 14307-14315.

111. T. Papalia, A. Barattucci, S. Campagna, F. Puntoriero, T. Salerno and P. Bonaccorsi, Org. Biomol. Chem., 2017, 15, 8211-8217.

112. A. L. Nguyen, K. E. Griffin, Z. Zhou, F. R. Fronczek, K. M. Smith and M. G. H. Vicente, New J. Chem., 2018, 42, 8241-8246.

113. F. Yan, K. Fan, Z. Bai, R. Zhang, F. Zu, J. Xu and X. Li, Trends Anal. Chem., 2017, 97, 15-35.

114. E. M. Nolan and S. J. Lippard, Acc. Chem. Res., 2009, 42, 193-203.

115. D. W. Domaille, E. L. Que and C. J. Chang, Nat. Chem. Biol., 2008, 4, 168-175.
116. S. Yoon, E. W. Miller, Q. He, P. H. Do and C. J. Chang, Angew. Chem. Int. Ed., 2007, 46, 6658-6661.

117. Y. J. Jang, E. J. Jun, Y. J. Lee, Y. S. Kim, J. S. Kim and J. Yoon, J. Org. Chem. 2005, 70, 9603-9606.

118. K. M. Swamy, Y. J. Lee, H. N. Lee, J. Chun, Y. Kim, S. J. Kim and J. Yoon, J. Org. Chem., 2006, 71, 8626-8628.

119. L. E. McQuade, J. Ma, G. Lowe, A. Ghatpande, A. Gelperin and S. J. Lippard, Proc. Natl. Acad. Sci. U.S.A., 2010, 107, 8525-8530.

120. J. Xie, M. Menand, S. Maisonneuve and R. Metivier, J. Org. Chem., 2007, 72, 5980-5985.

121. X. F. Zhang, J. Zhang and L. Liu, J. Fluoresc., 2014, 24, 819-826.

122. H. A. S. Al-Shamiri, M. T. H. A. Kana, I. M. Azzouz and A. H. M. Elwahy, Opt. Commun., 2010, 283, 1438-1444.

123. V. Dujols, F. Ford and A. W. Czarnik, J. Am. Chem. Soc., 1997, 119, 7386-7387.

124. M. Rajasekar, S. M. Khan, S. N. Devaraj and T. M. Das, Carbohydr. Res., 2011, 346, 1776-1785.

125. M. Rajasekar, R. Jegadeesh, N. Raaman and T. Mohan Das, Carbohydr. Res., 2011, 346, 2362-2367.

126. M. Rajasekar and T. M. Das, Carbohydr. Res., 2013, 379, 38-42.

127. M. Rajasekar and T. Mohan Das, J. Carbohydr. Chem., 2014, 33, 137151.

128. M. Rajasekar and T. M. Das, RSC Adv., 2014, 4, 42538-42545.

129. X. Chen, T. Pradhan, F. Wang, J. S. Kim and J. Yoon, Chem. Rev., 2012, 112, 1910-1956.

130. R. N. Dsouza, U. Pischel and W. M. Nau, Chem. Rev., 2011, 111, $7941-$ 7980.

131. M. Y. Berezin and S. Achilefu, Chem. Rev., 2010, 110, 2641-2684.

132. V. Bhalla, R. Tejpal and M. Kumar, Sens. Actuators B, 2010, 151, 180185.

133. J. Huang, Y. Xu and X. Qian, J. Org. Chem., 2009, 74, 2167-2170.

134. X. Chen, S. W. Nam, M. J. Jou, Y. Kim, S. J. Kim, S. Park and J. Yoon, Org. Lett., 2008, 10, 5235-5238.

135. E. M. Nolan and S. J. Lippard, J. Am. Chem. Soc., 2007, 129, 59105918.

136. A. Gupta and N. Kumar, RSC Adv., 2016, 6, 106413-106434.

137. M. Rajasekar and T. Mohan Das, RSC Adv., 2014, 4, 30976-30983.

138. K.-B. Li, H.-L. Zhang, B. Zhu, X.-P. He, J. Xie and G.-R. Chen, Dyes Pigm., 2014, 102, 273-277.

139. K. B. Li, Y. Zang, H. Wang, J. Li, G. R. Chen, T. D. James, X. P. He and H. Tian, Chem. Commun., 2014, 50, 11735-11737.

140. Z. Chen, W. Hu, M. Wang, L. Wang, G. Su and J. Wang, Carbohydr. Res., 2016, 429, 81-86.

141. W. T. Dou, Y. L. Zeng, Y. Lv, J. Wu, X. P. He, G. R. Chen and C. Tan, ACS Appl. Mater. Interfaces, 2016, 8, 13601-13606.

142. C. Z. Wang and X. P. He, Carbohydr. Res., 2018, 455, 1-4

143. G. Yang, X. Zhang, Z. Kochovski, Y. Zhang, B. Dai, F. Sakai, L. Jiang, Y. Lu, M. Ballauff, X. Li, C. Liu, G. Chen and M. Jiang, J. Am. Chem. Soc., 2016, 138, 1932-1937.

144. F. Sakai, G. Yang, M. S. Weiss, Y. Liu, G. Chen and M. Jiang, Nat. Commun., 2014, 5, 4634-4641.

145. A. Jo, J. Sung, S. Lee, H. Nam, H. W. Lee, J. Park, H. M. Kim, E. Kim and S. B. Park, Bioconjugate Chem., 2018, 29, 3394-3401.

146. T. Mosmann, J. Immunol. Meth., 1983, 65, 55-63.

147. C.-T. Chen, Chem. Mater., 2004, 16, 4389-4400.

148. Z. Guo, W. Zhu and H. Tian, Chem. Commun., 2012, 48, 6073-6084.

149. W. Zhu, X. Huang, Z. Guo, X. Wu, H. Yu and H. Tian, Chem. Commun., 2012, 48, 1784-1786.

150. M. Li, X. Wu, Y. Wang, Y. Li, W. Zhu and T. D. James, Chem. Commun., 2014, 50, 1751-1753.

151. D. Yu, Q. Zhang, S. Ding and G. Feng, RSC Adv., 2014, 4, 46561-46567.

152. K. Gu, Y. Xu, H. Li, Z. Guo, S. Zhu, S. Zhu, P. Shi, T. D. James, H. Tian and W. H. Zhu, J. Am. Chem. Soc., 2016, 138, 5334-5340.

153. J. Ma, J. Fan, H. Li, Q. Yao, J. Xia, J. Wang and X. Peng, Dyes Pigm., 2017, 138, 39-46.

154. D.-K. Ji, G.-R. Chen, X.-P. He and H. Tian, Adv. Funct. Mater., 2015, 25, 3483-3487. 
155. J. D. Zhang, J. Mei, X. L. Hu, X. P. He and H. Tian, Small, 2016, 12, 6562-6567.

156. D.-K. Ji, Y. Zhang, Y. Zang, W. Liu, X. Zhang, J. Li, G.-R. Chen, T. D. James and X.-P. He, J. Mater. Chem. B, 2015, 3, 9182-9185.

157. D. K. Ji, Y. Zhang, Y. Zang, J. Li, G. R. Chen, X. P. He and H. Tian, Adv. Mater., 2016, 28, 9356-9363.

158. C. Z. Wang, J. L. Chen, Y. Tang, Y. Zang, G. R. Chen, T. D. James, J. Li, C. Wu and X. P. He, ACS Appl. Mater. Interfaces, 2017, 9, 3272-3276.

159. H. H. Han, C. Z. Wang, Y. Zang, J. Li, T. D. James and X. P. He, Chem. Commun., 2017, 53, 9793-9796.

160. S. Cao, Z. Pei, Y. Xu and Y. Pei, Chem. Mater., 2016, 28, 4501-4506.

161. S. Chen, Y. Fang, Q. Zhu, W. Zhang, X. Zhang and W. Lu, RSC Adv. 2016, 6, 81894-81901.

162. J.-X. Song, X.-Y. Tang, D.-M. Zhou, W. Zhang, T. D. James, X.-P. He and H. Tian, Mater. Horiz., 2017, 4, 431-436.

163. S. S. Lucky, K. C. Soo and Y. Zhang, Chem. Rev., 2015, 115, 1990-2042.

164. S. Kwiatkowski, B. Knap, D. Przystupski, J. Saczko, E. Kedzierska, K. Knap-Czop, J. Kotlinska, O. Michel, K. Kotowski and J. Kulbacka, Biomed. Pharmacother., 2018, 106, 1098-1107.

165. X. Li, S. Lee and J. Yoon, Chem. Soc. Rev., 2018, 47, 1174-1188.

166. D. van Straten, V. Mashayekhi, H. S. de Bruijn, S. Oliveira and D. J. Robinson, Cancers, 2017, 9, 19.

167. Y. Shen, A. J. Shuhendler, D. Ye, J. J. Xu and H. Y. Chen, Chem. Soc. Rev., 2016, 45, 6725-6741.

168. Z. Zhou, J. Song, L. Nie and X. Chen, Chem. Soc. Rev., 2016, 45, 65976626.

169. R. Hudson and R. W. Boyle, J. Porph. Phthal., 2004, 08, 954-975.

170. J. M. Sutton, O. J. Clarke, N. Fernandez and R. W. Boyle, Bioconjugate Chem., 2002, 13, 249-263.

171. M. Ethirajan, Y. Chen, P. Joshi and R. K. Pandey, Chem. Soc. Rev., 2011, 40, 340-362.

172. A. E. O'Connor, W. M. Gallagher and A. T. Byrne, Photochem Photobiol., 2009, 85, 1053-1074.

173. O. Rusin, V. Kral, J. O. Escobedo and R. M. Strongin, Org. Lett., 2004, 6, 1373-1376.

174. A. Makky, J. P. Michel, A. Kasselouri, E. Briand, P. Maillard and V. Rosilio, Langmuir, 2010, 26, 12761-12768.

175. Y. Zorlu, M. A. Ermeydan, F. Dumoulin, V. Ahsen, H. Savoie and R. W. Boyle, Photochem. Photobiol. Sci., 2009, 8, 312-318.

176. M. A. Ermeydan, F. Dumoulin, T. V. Basova, D. Bouchu, A. G. Gürek, V. Ahsen and D. Lafont, New J. Chem., 2010, 34, 1153-1162.

177. Y. Zorlu, F. Dumoulin, D. Bouchu, V. Ahsen and D. Lafont, Tetrahedron Lett., 2010, 51, 6615-6618.

178. T. K. Horne and M. J. Cronje, J. Photochem. Photobiol. B, 2017, 173, 412-422.

179. F. Bachle, M. Hanack and T. Ziegler, Molecules, 2015, 20, 1836718386.

180. E. M. Garcia-Frutos and B. Gomez-Lor, J. Am. Chem. Soc., 2008, 130, 9173-9177.

181. W.-Y. Lai, R. Zhu, Q.-L. Fan, L.-T. Hou, Y. Cao and W. Huang, Macromolecules, 2006, 39, 3707-3709.

182. W. Y. Lai, Q. Y. He, R. Zhu, Q. Q. Chen and W. Huang, Adv. Funct. Mater., 2008, 18, 265-276.

183. M. Franceschin, L. Ginnari-Satriani, A. Alvino, G. Ortaggi and A. Bianco, Eur. J. Org. Chem., 2010, 134-141.

184. M. Talarico, R. Termine, E. M. García-Frutos, A. Omenat, J. L. Serrano, B. Gómez-Lor and A. Golemme, Chem. Mater., 2008, 20, 6589-6591.

185. U. Mitschke and P. Bäuerle, J. Mater. Chem., 2000, 10, 1471-1507.

186. S. Gunes, H. Neugebauer and N. S. Sariciftci, Chem. Rev., 2007, 107, 1324-1338.

187. J. Cremer and C. A. Briehn, Chem. Mater., 2007, 19, 4155-4165.

188. J. Feng, Y. Li and M. Yang, J. Polym. Sci. A, 2009, 47, 222-230.

189. K. R. Wang, Y. Q. Wang, H. W. An, J. C. Zhang and X. L. Li, Chem. Eur J., 2013, 19, 2903-2909.

190. K.-R. Wang, H.-W. An, D. Han, F. Qian and X.-L. Li, Chin. Chem. Lett., 2013, 24, 467-470.

191. G. Wang, X. Zhang, J. Geng, K. Li, D. Ding, K. Y. Pu, L. Cai, Y. H. Lai and B. Liu, Chem. Eur. J., 2012, 18, 9705-9713.
192. Q. Sun, H. Y. Zhu, J. F. Wang, X. Chen, K. R. Wang and X. L. Li, Bioorg. Chem., 2018, 79, 126-130.

193. I. Sarkar, A. Hemamalini, T. M. Das and A. K. Mishra, RSC Adv., 2016, 6, 27933-27943.

194. S. Goetz, M. Rejzek, S. A. Nepogodiev and R. A. Field, Carbohydr. Res., 2016, 433, 97-105.

195. T. Kanamori, A. Matsuyama, H. Naito, Y. Tsuga, Y. Ozako, S. I. Ogura, S. Okazaki and H. Yuasa, J. Org. Chem., 2018, 83, 13765-13775.

196. N. K. Singhal, A. Mitra, G. Rajsekhar, M. M. Shaikh, S. Kumar, P. Guionneau and C. P. Rao, Dalton Trans., 2009, 8432-8442.

197. S. Ou, Z. Lin, C. Duan, H. Zhang and Z. Bai, Chem. Commun., 2006, 4392-4394.

198. H. Yuasa, N. Fujii and S. Yamazaki, Org. Biomol. Chem., 2007, 5, 29202924.

199. H. Yuasa, N. Miyagawa, T. Izumi, M. Nakatani, M. Izumi and H. Hashimoto, Org. Lett., 2004, 6, 1489-1492.

200. Y.-J. Zhang, X.-P. He, M. Hu, Z. Li, X.-X. Shi and G.-R. Chen, Dyes Pigm., 2011, 88, 391-395.

201. D. M. Nguyen, A. Frazer, L. Rodriguez and K. D. Belfield, Chem. Mater., 2010, 22, 3472-3481.

202. A. Kumar and P. S. Pandey, Tetrahedron Lett., 2009, 50, 5842-5845.

203. H.-C. Hung, C.-W. Cheng, Y.-Y. Wang, Y.-J. Chen and W.-S. Chung, Eur. J. Org. Chem., 2009, 2009, 6360-6366.

204. H.-C. Hung, C.-W. Cheng, I. T. Ho and W.-S. Chung, Tetrahedron Lett., 2009, 50, 302-305.

205. X.-P. He, Z. Song, Z.-Z. Wang, X.-X. Shi, K. Chen and G.-R. Chen, Tetrahedron, 2011, 67, 3343-3347.

206. H. Masuhara, H. Shioyama, T. Saito, K. Hamada, S. Yasoshima and N. Mataga, J. Phys. Chem., 1984, 88, 5868-5873.

207. D. T. Shi, X. L. Wei, Y. Sheng, Y. Zang, X. P. He, J. Xie, G. Liu, Y. Tang, J. Li and G. R. Chen, Sci. Rep., 2014, 4, 4252-4257.

208. J. L. Xue, X. P. He, J. W. Yang, D. T. Shi, C. Y. Cheng, J. Xie, G. R. Chen and K. Chen, Carbohydr. Res., 2012, 363, 38-42.

209. Z. Song, X.-P. He, X.-P. Jin, L.-X. Gao, L. Sheng, Y.-B. Zhou, J. Li and G.R. Chen, Tetrahedron Lett., 2011, 52, 894-898.

210. Y. H. Tang, Y. Qu, Z. Song, X. P. He, J. Xie, J. Hua and G. R. Chen, Org. Biomol. Chem., 2012, 10, 555-560.

211. E. Bokor, S. Kun, D. Goyard, M. Toth, J. P. Praly, S. Vidal and L. Somsak, Chem. Rev., 2017, 117, 1687-1764.

212. X. He, J. Xie, G. Chen and K. Chen, Chin. J. Chem., 2012, 30, 28742878.

213. C. Lin, S. Maisonneuve, R. Metivier and J. Xie, Chem. Eur. J., 2017, 23, 14996-15001.

214. G. Despras, J. Hain and S. O. Jaeschke, Chem. Eur. J., 2017, 23, 1083810847.

215. J. Hain and G. Despras, Chem. Commun., 2018, 54, 8563-8566.

216. Z. Jia, R. K. Singh and D. Wang, Biomedical Applications of "Click"Modified Cyclodextrins in Click Chemistry in Glycoscience: New Developments and Strategies, eds. Z. J. Witczak and R. Bielski, Wiley, Hoboken, 2013, Chap. 11, pp. 271-292.

217. M. E. Brewster and T. Loftsson, Adv. Drug Deliv. Rev., 2007, 59, 645666.

218. J. R. Kanwar, B. M. Long and R. K. Kanwar, Curr. Med. Chem., 2011, 18, 2079-2085.

219. J. Zhang and P. X. Ma, Adv. Drug Deliv. Rev., 2013, 65, 1215-1233.

220. A. R. Khan, P. Forgo, K. J. Stine and V. T. D'Souza, Chem. Rev., 1998, 98, 1977-1996.

221. B. Wang, E. Zaborova, S. Guieu, M. Petrillo, M. Guitet, Y. Bleriot, M. Menand, Y. Zhang and M. Sollogoub, Nat. Commun., 2014, 5, 5354.

222. M. Sollogoub, Eur. J. Org. Chem., 2009, 1295-1303.

223. P. Shahgaldian and U. Pieles, Sensors, 2006, 6, 593-615.

224. V. K. Tiwari, B. B. Mishra, K. B. Mishra, N. Mishra, A. S. Singh and X. Chen, Chem. Rev., 2016, 116, 3086-3240.

225. M. E. Davis and M. E. Brewster, Nat. Rev. Drug Discov., 2004, 3, $1023-$ 1035.

226. O. David, S. Maisonneuve and J. Xie, Tetrahedron Lett., 2007, 48, 6527-6530 
227. V. Souchon, S. Maisonneuve, O. David, I. Leray, J. Xie and B. Valeur, Photochem. Photobiol. Sci., 2008, 7, 1323-1331.

228. S. Maisonneuve, Q. Fang and J. Xie, Tetrahedron, 2008, 64, 87168720.

229. W. Wang, Y. Zhang, Q. Yang, M. Sun, X. Fei, Y. Song, Y. Zhang and Y. Li, Nanoscale, 2013, 5, 4958-4965.

230. X.-P. He, R.-H. Li, S. Maisonneuve, Y. Ruan, G.-R. Chen and J. Xie, Chem. Commun., 2014, 50, 14141-14144.

231. G. Cutrone, G. Benkovics, M. Malanga, J. M. Casas-Solvas, E. Fenyvesi, S. Sortino, L. Garcia-Fuentes and A. Vargas-Berenguel, Carbohydr. Polym., 2018, 199, 649-660.

232. R. Corradini, C. Paganuzzi, R. Marchelli, S. PagliariPresent address: Callegari, S. Sforza, A. Dossena, G. Galaverna and A. Duchateau, J. Mater. Chem., 2005, 15, 2741-2746.

233. S. Chaudhuri, D. J. DiScenza, B. Smith, R. Yocum and M. Levine, New J. Chem., 2017, 41, 14431-14437.

234. A. Anilkumar, T. E. Gopala Krishna Murthy and A. P. Rani, Ind. J. Pharm. Educ. Res., 2016, 50, 665-675.

235. J. Lai, B. P. Shah, E. Garfunkel and K. B. Lee, ACS Nano, 2013, 7, 27412750.

236. R. Sunasee and R. Narain, Macromol. Biosci., 2013, 13, 9-27.

237. L. L. Kiessling and J. C. Grim, Chem. Soc. Rev., 2013, 42, 4476-4491.

238. S. G. Spain and N. R. Cameron, Polym. Chem., 2011, 2, 60-68.

239. R. Sunasee, C. K. Adokoh, J. Darkwa and R. Narain, Expert Opin. Drug Deliv., 2014, 11, 867-884.

240. E. J. Gordon, L. E. Strong and L. L. Kiessling, Bioorg. Med. Chem., 1998, 6, 1293-1299.

241. T. Yoshida, T. Akasaka, Y. Choi, K. Hattori, B. Yu, T. Mimura, Y. Kaneko, H. Nakashima, E. Aragaki, M. Premanathan, N. Yamamoto and T. Uryu, J. Polym. Sci. A, 1999, 37, 789-800.

242. R. Roy and M.-G. Baek, Rev. Mol. Biotechnol., 2002, 90, 291-309.

243. C. Fleming, A. Maldjian, D. Da Costa, A. K. Rullay, D. M. Haddleton, J. St John, P. Penny, R. C. Noble, N. R. Cameron and B. G. Davis, Nat. Chem. Biol., 2005, 1, 270-274.

244. P. K. Dhal, S. R. Holmes-Farley, C. C. Huval and T. H. Jozefiak, Polymers as Drugs in Polymer Therapeutics I. Advances in Polymer Science, eds. R. Satchi-Fainaro and R. Duncan, Springer, Berlin, 2006, vol. 192, pp. 9-58.

245. J. Li, S. Zacharek, X. Chen, J. Wang, W. Zhang, A. Janczuk and P. G. Wang, Bioorg. Med. Chem., 1999, 7, 1549-1558.

246. A. C. Roche, I. Fajac, S. Grosse, N. Frison, C. Rondanino, R. Mayer and M. Monsigny, Cell. Mol. Life Sci., 2003, 60, 288-297.

247. Y. H. Yun, D. J. Goetz, P. Yellen and W. Chen, Biomaterials, 2004, 25 147-157.

248. G. Moad, E. Rizzardo and S. H. Thang, Aust. J. Chem., 2005, 58, 379410.

249. S. Perrier and P. Takolpuckdee, J. Polym. Sci. A, 2005, 43, 5347-5393.

250. G. Moad, Aust. J. Chem., 2006, 59, 661-662.

251. K. Matyjaszewski, Science, 2011, 333, 1104-1105.

252. W. A. Braunecker and K. Matyjaszewski, Progress Polym. Sci., 2007, 32, 93-146.

253. A. Ghadban and L. Albertin, Polymers, 2013, 5, 431-526.

254. V. Vazquez-Dorbatt, J. Lee, E. W. Lin and H. D. Maynard, ChemBioChem, 2012, 13, 2478-2487.

255. J.-S. Wang and K. Matyjaszewski, J. Am. Chem. Soc., 1995, 117, 56145615.

256. Q. Chen, Y. Cui, J. Cao and B.-H. Han, Polymer, 2011, 52, 383-390.

257. R. L. Phillips, I. B. Kim, L. M. Tolbert and U. H. Bunz, J. Am. Chem. Soc., 2008, 130, 6952-6954.

258. M. D. Disney, J. Zheng, T. M. Swager and P. H. Seeberger, J. Am. Chem. Soc., 2004, 126, 13343-13346.

259. M. Zhang, P. Wu, W. T. Dou, H. H. Han, X. P. He, C. Tan and Y. Jiang, Chem. Commun., 2017, 53, 5625-5628.

260. J. Shi, L. Cai, K. Y. Pu and B. Liu, Chem. Asian J., 2010, 5, 301-308.

261. P. Sun, Y. He, M. Lin, Y. Zhao, Y. Ding, G. Chen and M. Jiang, ACS Macro Lett., 2013, 3, 96-101.

262. C. Xue, S. P. Jog, P. Murthy and H. Liu, Biomacromolecules, 2006, 7, 2470-2474.
263. C. Xue, S. Velayudham, S. Johnson, R. Saha, A. Smith, W. Brewer, P. Murthy, S. T. Bagley and H. Liu, Chem. Eur. J., 2009, 15, 2289-2295.

264. K. Petkau, A. Kaeser, I. Fischer, L. Brunsveld and A. P. Schenning, J. Am. Chem. Soc., 2011, 133, 17063-17071.

265. F. L. Thorp-Greenwood, M. P. Coogan, L. Mishra, N. Kumari, G. Rai and S. Saripella, New J. Chem., 2012, 36, 64-72.

266. Q. Zhao, C. Huang and F. Li, Chem. Soc. Rev., 2011, 40, 2508-2524.

267. F. L. Thorp-Greenwood, R. G. Balasingham and M. P. Coogan, J. Organomet. Chem., 2012, 714, 12-21.

268. K. K.-W. Lo, M.-W. Louie and K. Y. Zhang, Coord. Chem. Rev., 2010, 254, 2603-2622.

269. M.-W. Louie, H.-W. Liu, M. H.-C. Lam, T.-C. Lau and K. K.-W. Lo, Organometallics, 2009, 28, 4297-4307.

270. K. K. Lo, M. W. Louie, K. S. Sze and J. S. Lau, Inorg. Chem., 2008, 47, 602-611.

271. K. Kam-Wing Lo, D. Chun-Ming Ng, W.-K. Hui and K.-K. Cheung, J. Chem. Soc., Dalton Trans., 2001, 2634-2640.

272. V. Fernandez-Moreira, F. L. Thorp-Greenwood and M. P. Coogan, Chem. Commun., 2010, 46, 186-202.

273. D. Septiadi, A. Aliprandi, M. Mauro and L. De Cola, RSC Adv., 2014, 4, 25709-25718.

274. W. H. Kim, J. Lee, D. W. Jung and D. R. Williams, Sensors, 2012, 12, 5005-5027.

275. J. J. Chen, J. Jing, H. Chang, Y. Rong, Y. Hai, J. Tang, J. L. Zhang and P. Xu, Autophagy, 2013, 9, 894-904.

276. D. Grunstein, M. Maglinao, R. Kikkeri, M. Collot, K. Barylyuk, B. Lepenies, F. Kamena, R. Zenobi and P. H. Seeberger, J. Am. Chem. Soc., 2011, 133, 13957-13966.

277. R. Kikkeri, X. Liu, A. Adibekian, Y. H. Tsai and P. H. Seeberger, Chem. Commun., 2010, 46, 2197-2199.

278. M. Gottschaldt, U. S. Schubert, S. Rau, S. Yano, J. G. Vos, T. Kroll, J. Clement and I. Hilger, ChemBioChem, 2010, 11, 649-652.

279. M.-J. Li, P. Jiao, W. He, C. Yi, C.-W. Li, X. Chen, G.-N. Chen and M. Yang, Eur. J. Inorg. Chem., 2011, 197-200.

280. W. H. Law, L. C. Lee, M. W. Louie, H. W. Liu, T. W. Ang and K. K. Lo, Inorg. Chem., 2013, 52, 13029-13041.

281. D. L. Ma, T. Y. Shum, F. Zhang, C. M. Che and M. Yang, Chem. Commun., 2005, 2005, 4675-4677.

282. M. W. Louie, H. W. Liu, M. H. Lam, Y. W. Lam and K. K. Lo, Chem. Eur. J., 2011, 17, 8304-8308.

283. T. Okada, T. Makino and N. Minoura, Bioconjugate Chem., 2009, 20, 1296-1298.

284. T. Okada and N. Minoura, J. Biomed. Opt., 2011, 16, 037001-037006.

285. R. Roy and J. M. Kim, Tetrahedron, 2003, 59, 3881-3893.

286. P. Reeh and J. de Mendoza, Chem. Eur. J., 2013, 19, 5259-5262.

287. P. T. Corbett, J. Leclaire, L. Vial, K. R. West, J. L. Wietor, J. K. Sanders and S. Otto, Chem. Rev., 2006, 106, 3652-3711.

288. A. Palmioli, A. Aliprandi, D. Septiadi, M. Mauro, A. Bernardi, L. De Cola and M. Panigati, Org. Biomol. Chem., 2017, 15, 1686-1699.

289. D. Donghi, G. D'Alfonso, M. Mauro, M. Panigati, P. Mercandelli, A. Sironi, P. Mussini and L. D'Alfonso, Inorg. Chem., 2008, 47, 4243-4255.

290. C. Mari, M. Panigati, L. D'Alfonso, I. Zanoni, D. Donghi, L. Sironi, M. Collini, S. Maiorana, C. Baldoli, G. D'Alfonso and E. Licandro, Organometallics, 2012, 31, 5918-5928.

291. E. Ferri, D. Donghi, M. Panigati, G. Prencipe, L. D'Alfonso, I. Zanoni, C. Baldoli, S. Maiorana, G. D'Alfonso and E. Licandro, Chem. Commun., 2010, 46, 6255-6257.

292. B. R. Griffith, J. M. Langenhan and J. S. Thorson, Curr. Opin. Biotechnol., 2005, 16, 622-630.

293. J. M. Langenhan, B. R. Griffith and J. S. Thorson, J. Nat. Prod., 2005, 68, 1696-1711.

294. A. Palmioli, M. Panigati and A. Bernardi, Org. Biomol. Chem., 2018, 16, 8413-8419.

295. A. Palmioli and B. La Ferla, Org. Lett., 2018, 20, 3509-3512.

296. D. A. Giljohann and C. A. Mirkin, Nature, 2009, 462, 461-464.

297. M. A. Dobrovolskaia and S. E. McNeil, Nat. Nanotechnol., 2007, 2, 469-478. 
298. R. A. Sperling, P. Rivera Gil, F. Zhang, M. Zanella and W. J. Parak, Chem. Soc. Rev., 2008, 37, 1896-1908.

299. J. M. de la Fuente and S. Penades, Biochim. Biophys. Acta, 2006, 1760, 636-651.

300. K. El-Boubbou, C. Gruden and X. Huang, J. Am. Chem. Soc., 2007, 129, 13392-13393.

301. J. Zheng, C. Zhou, M. Yu and J. Liu, Nanoscale, 2012, 4, 4073-4083.

302. C. C. Huang, C. T. Chen, Y. C. Shiang, Z. H. Lin and H. T. Chang, Anal. Chem., 2009, 81, 875-882.

303. M. Zheng and X. Huang, J. Am. Chem. Soc., 2004, 126, 12047-12054.

304. C. C. Huang, S. H. Chiu, Y. F. Huang and H. T. Chang, Anal. Chem., 2007, 79, 4798-4804.

305. X. Wang, O. Ramstrom and M. Yan, Anal. Chem., 2010, 82, 9082-9089.

306. C. Yung-Chi and W. H. Prusoff, Biochem. Pharmacol., 1973, 22, 30993108.
307. X. Le Guevel, M. Perez Perrino, T. D. Fernandez, F. Palomares, M. J. Torres, M. Blanca, J. Rojo and C. Mayorga, ACS Appl. Mater. Interfaces, 2015, 7, 20945-20956.

308. Z. Luo, X. Yuan, Y. Yu, Q. Zhang, D. T. Leong, J. Y. Lee and J. Xie, J. Am. Chem. Soc., 2012, 134, 16662-16670.

309. X. Jia, J. Li and E. Wang, Small, 2013, 9, 3873-3879.

310. B. Tang, L. Cao, K. Xu, L. Zhuo, J. Ge, Q. Li and L. Yu, Chem. Eur. J., 2008, 14, 3637-3644.

311. Y. Guo, C. Sakonsinsiri, I. Nehlmeier, M. A. Fascione, H. Zhang, W. Wang, S. Pohlmann, W. B. Turnbull and D. Zhou, Angew. Chem. Int. Ed., 2016, 55, 4738-4742.

312. B. Schmidt, S. Sankaran, L. Stegemann, C. A. Strassert, P. Jonkheijm and J. Voskuhl, J. Mater. Chem. B, 2016, 4, 4732-4738. 